\title{
TOWARDS AN AUTOMATED WEIGHT LIFTING COACH: INTRODUCING LIFT
}

\author{
A Thesis \\ presented to \\ the Faculty of California Polytechnic State University \\ San Luis Obispo
}

\author{
In Partial Fulfillment \\ of the Requirements for the Degree \\ Master of Science in Computer Science
}

by

Michael Lady

June 2014 
(C) 2014

Michael Lady

ALL RIGHTS RESERVED 


\section{COMMITTEE MEMBERSHIP}

TITLE:

Towards an automated weight lifting coach: introducing LIFT

AUTHOR:

Michael Lady

DATE SUBMITTED: June 2014

COMMITTEE CHAIR: Christopher Lupo, Ph.D.

Assistant Professor of Computer Science

COMmitTeE MembeR: Alexander Dekhtyar, Ph.D.

Professor of Computer Science

COMMITTEE MEMBER: Lynne Slivovsky, Ph.D.

Professor of Electrical Engineering 


\begin{abstract}
Towards an automated weight lifting coach: introducing LIFT
\end{abstract}

Michael Lady

The fitness device market is young and rapidly growing. More people than ever before take count of how many steps they walk, how many calories they burn, their heart rate over time, and even their quality of sleep. New, and as of yet, unreleased fitness devices have promised the next evolution of functionality with exercise technique analysis. These next generation of fitness devices have wrist and armband style form factors, which may not be optimal for barbell exercises such as back squat, bench press, and overhead press where a sensor on one arm may not provide the most relevant data about a lift.

Barbell path analysis is a well-known visual tool to help diagnose weightlifting technique deficiencies, but requires a camera pointed at the athlete that is integrated with motion-tracking software. This camera set up is not available at most gyms, so this motivates the use of a small, unobtrusive sensor to obtain data about an athlete's weightlifting technique. Researchers have shown that an accelerometer attached to a barbell while the athlete is lifting yields just as accurate acceleration information as a camera [96]. The LIFT (Leveraging Information For Training) automated weight lifting coach attempts to implement a simple, unobtrusive system for analyzing and providing feedback on barbell weight lifting technique. 


\section{ACKNOWLEDGMENTS}

First off, a very special thank you to the Cal Poly Athletic Weight Room and Cal Poly ASI Recreation Center for allowing me to conduct my experiment in their state-of-the-art facilities. The staff were very welcoming and open to the idea of incorporating technology into the weight room. Cameron Van Wye of the Athletic Weight Room in particular has been an invaluable resource for recruiting healthy Cal Poly athletes to be test subjects to provide real-world, hard-fought, yet safely executed exercise data.

Thank you to my lead thesis adviser, Dr. Lupo for consistently giving me feedback on my progress and being there to bounce ideas off of. Thank you to my other thesis committee members, Dr. Dekhtyar, and Dr. Slivovsky. Without taking Dr. Dekhtyar's class in data mining, I would not have had the background necessary to successfully execute this project. Dr. Slivovsky's domain expertise in her hobby of powerlifting was invaluable in receiving feedback for this project.

Thank you to my Mom, Dad, and brother. They have supported me throughout my college career and patiently listened to me drone on about technology ever since I could use a computer.

Thank you to my friends, most of whom are either technically proficient in writing software or Brazilian jiu-jitsu. You guys have been my extended family and have made me feel at home while away from home. 


\section{TABLE OF CONTENTS}

List of Tables $\quad$ ix

List of Figures $\quad$ X

1 Introduction 1

1.1 Contributions ........................ 1

1.2 Outline ....................... 2

2 Domain Background 3

2.1 Benefits of compound exercise . . . . . . . . . . . . . . 3

2.2 Benefits of barbell exercise . . . . . . . . . . . . . . . 4

2.3 Benefits of compound barbell exercise . . . . . . . . . . 5 5

2.4 Weight lifting technique . . . . . . . . . . . . . 7

2.4.1 Back Squat .................. 9

2.4.1.1 Correct technique . . . . . . . . . . . 10

2.4.1.2 Incorrect technique . . . . . . . . . . . . 11

2.4.2 Bench Press . . . . . . . . . . . . . . . . 15

2.4.2.1 Correct technique . . . . . . . . . . 16

2.4.2.2 Incorrect technique . . . . . . . . . . 17

2.4.3 Overhead Press . . . . . . . . . . . . . . 20

2.4.3.1 Correct technique . . . . . . . . . . . 20

2.4.3.2 Incorrect technique . . . . . . . . . . . . 20

2.5 Weight lifting technique feedback . . . . . . . . . . . 23

2.5.1 Visual feedback . . . . . . . . . . . . . . . . . 24

2.5.2 Wearable devices . . . . . . . . . . . . 26

3 Technical Background 30

3.1 How device sensors work . . . . . . . . . . . . . . 30

3.1.1 Accelerometer . . . . . . . . . . . . . 30 
3.1 .2 Gyroscope . . . . . . . . . . . . . . . . . . . 33

3.1 .3 Magnetometer . . . . . . . . . . . . . . . . . . . 35

3.2 Combining sensor data together . . . . . . . . . . . . 36

3.3 How data classification systems work . . . . . . . . . . . . 39

3.3 .1 Decision tree . . . . . . . . . . . . . . . . . . 40

3.3.1.1 Decision Tree Algorithm . . . . . . . . . . . . . . 41

3.3.1.2 Classifier Evaluation . . . . . . . . . . . . . 43

3.3.2 Random forest . . . . . . . . . . . . . . . . . . . . 44

3.3 .3 Extra-tress . . . . . . . . . . . . . . . . 45

4 Related Work 48

4.1 General motion tracking systems . . . . . . . . . . . . 48

4.2 Systems used to augment healthcare . . . . . . . . . . . . 49

4.3 Systems used to augment general exercise performance . . . . . 50

4.4 Systems used to augment weight lifting performance . . . . . . . 51

5 Methodology 54

$5.1 \quad$ Barbell Lifts . . . . . . . . . . . . . . . . . . . . . . . . . 54

$5.2 \quad$ Subject testing $\ldots \ldots \ldots \ldots \ldots \ldots \ldots \ldots \ldots$

5.2 .1 Profile of subjects . . . . . . . . . . . . . 55

5.2 .2 Realistic technique deficiencies . . . . . . . . . . 56

6 Implementation $\quad 57$

6.1 Data collection . . . . . . . . . . . . . . . . . 57

6.2 Technique classification . . . . . . . . . . . . . . . . 60

6.3 Automated weight lifting coach $\ldots \ldots \ldots \ldots$

7 Experimental Framework $\quad 65$

7.1 Research protocol . . . . . . . . . . . . . . . . . 65

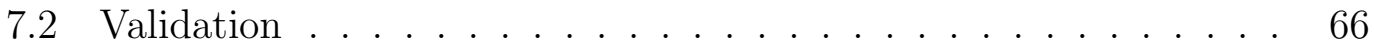

8 Results 67

8.1 Squat results . . . . . . . . . . . . . . . . . . . 68

8.2 Bench press results . . . . . . . . . . . . . . . . . 72

8.3 Overhead press results . . . . . . . . . . . . . 76 
9 Analysis 85

9.1 Research study bias and error . . . . . . . . . . . . . . 85

9.2 Athlete bias . . . . . . . . . . . . . . . . 85

9.3 Technique deficiency challenges . . . . . . . . . . . 86

9.4 Researcher error . . . . . . . . . . . . . . . . 86

9.5 Overcoming research study bias and error . . . . . . . . . . . . 87

9.6 Technical error considerations . . . . . . . . . . . . . . 87

9.6.1 Orientation taring. . . . . . . . . . . . . 87

9.6.2 Magnetometer effects . . . . . . . . . . . . . 88

9.6.3 Euler angles and Gimbal lock . . . . . . . . . . . . . 90

9.6.4 Data collection rate . . . . . . . . . . . . . 91

9.6.5 Exercise technique deficiency ambiguity . . . . . . . . . . 92

10 Conclusions 93

11 Future Work $\quad 94$

11.1 Visual feedback . . . . . . . . . . . . . . . . . . . 94

11.2 Repetition detection . . . . . . . . . . . . . . . . . . 95

11.3 Sensor compatibility . . . . . . . . . . . . . . . 95

11.4 Body type clustering . . . . . . . . . . . . . . . . 96

11.5 Female study . . . . . . . . . . . . . . . . . . . . . 96

11.6 Alternative ways to provide weight lifting feedback . . . . . . . 97

$\begin{array}{lr}\text { Bibliography } & 98\end{array}$ 


\section{LIST OF TABLES}

3.1 How quickly error can accrue over time due to incorrect initial orientation calculations $[26] \ldots \ldots 33$

8.1 Comparison of squat measures between v1 and v2 . . . . . . . 71

8.2 Squat label number table . . . . . . . . . . . . . . . . 72

8.3 Comparison of bench press measures between v1 and v2 . . . . 77

8.4 Bench press label numbers . . . . . . . . . . . . . . . 78

8.5 Comparison of overhead press measures between v1 and v2 . . . . 80

8.6 Overhead press v1 label numbers . . . . . . . . . . . . . 82 


\section{LIST OF FIGURES}

2.1 How the body gets stronger $[102] \ldots \ldots \ldots \ldots$

2.2 Training complexity over time $[90] \ldots \ldots \ldots \ldots$

2.3 Socrates' perspective on fitness $[50] \ldots \ldots \ldots \ldots$

2.4 How the body is balanced during standing barbell exercises [90] . 9

2.5 The correct way to squat $[90] \ldots \ldots \ldots \ldots$

2.6 The correct way to bench press $[90] \ldots \ldots \ldots \ldots$

2.7 Anatomy of a bench press with elbows at 45 degrees on the left and 90 degrees on the right. [90] . . . . . . . . . . . 19

2.8 The correct way to overhead press $[90] \ldots \ldots \ldots$

2.9 The importance of getting under the bar during the press to keep it balanced over the mid foot $[90] \ldots \ldots$. . . . . . . 23

2.10 Keeping wrists straight to avoid wasting energy [90] . . . . . . . . 24

2.11 Using an inefficient bar path for the press $[90] \ldots \ldots \ldots . . .25$

2.12 Visual bar path feedback $[61] \ldots \ldots \ldots$

2.13 A snapshot of the wearable device market $[60] \ldots \ldots$. . . . . 27

2.14 Training feedback device competitors $[2,3,11,16] \ldots$. . . . . . . 29

3.1 A model of how an accelerometer measures acceleration [93] . . 32

3.2 A model of how a gyroscope measures angular velocity [46] . . . 34

3.3 A model of how a magnetometer measures the magnetic field [35] 36

3.4 An example of how the magnetic field can be altered by a ferrous metal $[25] \ldots \ldots \ldots \ldots \ldots$

3.5 An example of the output of a Kalman filter given accelerometer and gyroscope signals $[27] \ldots \ldots \ldots$

3.6 Verbal example of a decision tree $[89] \ldots \ldots \ldots \ldots$

3.7 C4.5 algorithm for decision tree induction [41] . . . . . . . . 41 
3.8 selectSplittingAttribute() functions using information gain and information gain ratio measures. [41] . . . . . . . . . . . . . 42

3.9 A visual example of a decision tree [113] . . . . . . . . . . . 43

3.10 Random Forest Pseudocode $[65] \ldots \ldots$. . . . . . . . 45

3.11 Extra-Trees splitting algorithm $[47] \ldots \ldots \ldots \ldots$

6.1 A system diagram of the YEI IMU $[21] \ldots \ldots \ldots \ldots$

6.2 A system diagram of the data collection phase of LIFT . . . . . 59

6.3 The axes of the YEI IMU when placed on a barbell . . . . . . . 61

6.4 A system diagram of the technique learning phase of LIFT . . . 62

6.5 A system diagram of LIFT's future automated technique feedback

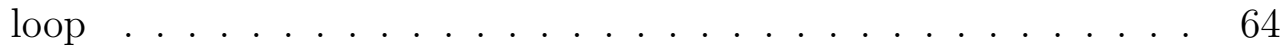

8.1 Full normalized confusion matrix for v1 squat . . . . . . . . 71

8.2 Simple normalized confusion matrix for v1 squat . . . . . . . 73

8.3 Full normalized confusion matrix for v2 squat . . . . . . . . . 74

8.4 Simple normalized confusion matrix for v2 squat . . . . . . . . 75

8.5 Full normalized confusion matrix for v1 bench press . . . . . 77

8.6 Simple normalized confusion matrix for v1 bench press . . . . . 79

8.7 Full normalized confusion matrix for v2 bench press _ . . . . 80

8.8 Simple normalized confusion matrix for v2 bench press . . . . . 81

8.9 Full normalized confusion matrix for v1 overhead press _ . . . . 83

8.10 Simple normalized confusion matrix for v1 overhead press . . . . 84

9.1 The second design of LIFT's sensor packaging . . . . . . . . . 90

9.2 The ambiguity between the yaw (pink) and roll (green) degrees of freedom $[39] \ldots \ldots$. . . . . . . . . . . . . . . . . . . . . . . . 91

11.1 Magnetometer measurements (bottom) correlates nicely with when repetitons occured . . . . . . . . . . . . . . . . 95 


\title{
CHAPTER 1
}

\author{
Introduction
}

The Quantified Self (QS) movement [114] is a rapidly growing trend that aims to help people keep track of various aspects of their lives to facilitate personal development. One of the hot areas of QS is health and fitness $[17,19]$. Technology that counts how many steps users walk and calories they burn used to be limited to specialized pedometer devices, but since accelerometers and gyroscopes have become cheaper in recent years due to manufacturing improvements $[108,112]$ to keep up with the demand in the smartphone industry, the technology is now ubiquitous. The fitness industry is now saturated with these tracking devices in many different form factors. New fitness devices are on the horizon to reveal even more detailed information about a user's health by giving the user feedback about how they are performing specific exercises.

\subsection{Contributions}

The LIFT automated weight lifting coach proposed in this paper aims to be an open-source, barbell-centric analysis system that is a complement to proprietary, on-body fitness devices. The LIFT weight lifting coach is modular and extensible enough to be compatible with future fitness devices and their enhanced motiontracking capabilities. 


\subsection{Outline}

The paper is laid out as follows: Chapter 2 covers the necessary domain-specific background information; Chapter 3 covers the technical background information; Chapter 4 covers related movement tracking system research; Chapter 5 covers the methodology of the LIFT automated weight lifting coach; Chapter 6 covers the implementation of the LIFT automated weight lifting coach; Chapter 7 covers an experimental user study framework for the LIFT automated weight lifting coach; Chapter 8 covers the results of the user study; Chapter 9 covers the conclusions about the current capabilities of the LIFT automated weight lifting coach; Chapter 10 looks forward to future work to be done on the LIFT automated weight lifting coach. 


\section{CHAPTER 2}

\section{Domain Background}

Barbell weight lifting is a very effective method for increasing overall strength in athletes, which in turn, will increase their performance and decrease risk of injury in their particular sport $[44,63,90]$. Mark Rippetoe is a well-known strength and conditioning coach, and author of one of the most well-regarded and technically thorough manuals on barbell training, Starting Strength $[42,56,90]$. Rippetoe states that all people can benefit from becoming stronger due to a simple fact of life that stronger people live longer $[40,90]$. Rippetoe's thesis is that the simplest way one can increase physical strength is to progressively add weight to compound barbell exercises [90].

\subsection{Benefits of compound exercise}

Compound exercises are exercises that involve more than one joint and muscle group, such as squat, bench press, and overhead press [90]. This is opposed to isolation exercises that only involve one joint and muscle group, such as bicep curls and leg extension machines [90]. The compound type of exercise forces many muscle groups to work together to complete a given movement [90].

"Real world" strength-oriented tasks, such as lifting a couch or carrying a box, are multi-joint, or "compound", dynamic movements. Dynamic movements involve contracting muscles in a continuous eccentric and concentric fashion [54]. 
Virtually all athletic, sport-specific movements are compound, dynamic movements [90]. Therefore, training in this same compound, dynamic fashion will best prepare a trainee, weekend warrior and athlete alike, for the real world and sport-specific activity [90].

\subsection{Benefits of barbell exercise}

The advantage of training with barbells is that they can be gradually loaded as a trainee's strength progresses. Dumbbells, kettle bells, and other resistance training implements do not have as fine of granularity of progress as barbells do.

Barbells have plates that can load as little as $0.25 \mathrm{lbs}$, whereas individual dumbbells traditionally only increase in weight $5 \mathrm{lbs}$ at a time [73]. For bilateral exercises (where both sides of the body are used at the same time), such as the bench press, this 5 lbs increase for dumbbells is actually realized by both limbs as a total of $10 \mathrm{lbs}$ increase in weight when compared to bench pressing with a barbell. This is a significant jump (10\%) for a novice male trainee whose bench press 1 repetition maximum may only be 100 lbs. The corresponding smallest increase in weight for a barbell would only be $0.5 \mathrm{lbs}$, which is only a $0.5 \%$ more than their previous best lift and is much more attainable workout-to-workout progress [71].

A novice trainee can sustain this gradual increase in workout-to-workout weight, or "progressive overload" for a relatively long period of time [72]. This is because their body is able to recover from the physiological stress of the loaded exercise and "supercompensate" for the next time the load will be encountered, as seen in Figure 2.1 [102]. The trainee is no longer considered to be a novice when they are unable to consistently add incremental weight to their lifts on a 
weekly basis [72]. The newly-minted intermediate trainee must move on to a more advanced training regimen following the timeline laid out in Figure 2.2 to avoid stagnation or "hitting a plateau" on their lifts [72].

\section{GRAPH I}

\section{Ideal workout and recovery situation from single workout}

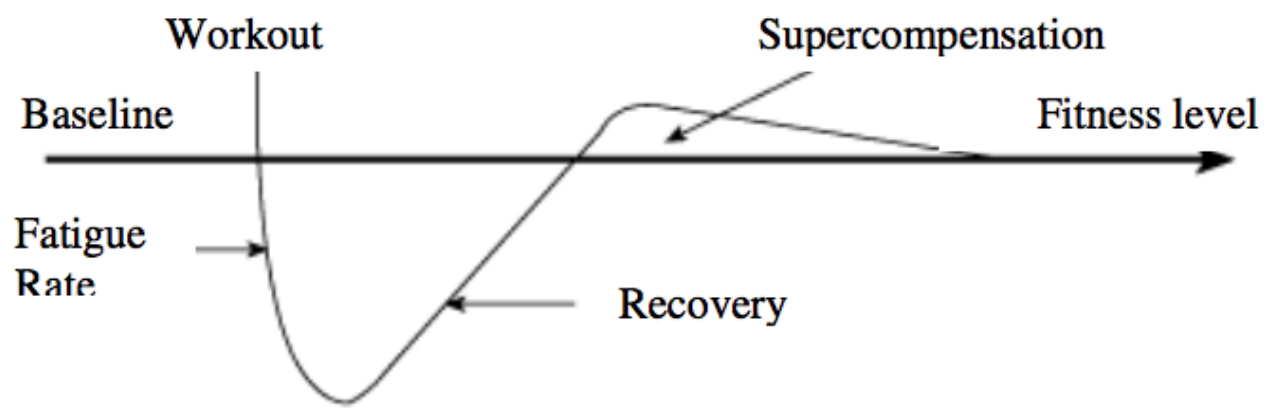

Figure 2.1: How the body gets stronger [102]

\subsection{Benefits of compound barbell exercise}

Compound exercises performed with barbells showcase the beauty of progressive overload: even the most novice of trainees can be gradually molded over time to becoming an overall stronger version of themselves. This fact is what motivates this study of automating barbell weight lifting technique feedback; the consequences of enabling more widespread, safe use of compound barbell exercises can have far-reaching impacts not only in the realm of sports performance, but also on the health and well-being of the general population as a whole.

Contrary to popular belief, weight lifting does not necessarily make one "musclebound" and inflexible [90]. On the contrary, barbell weight lifting through a full range of motion promotes flexibility [79]. Trainees who are not able to lift weights through a full range of motion or have pre-existing injuries are encouraged to 


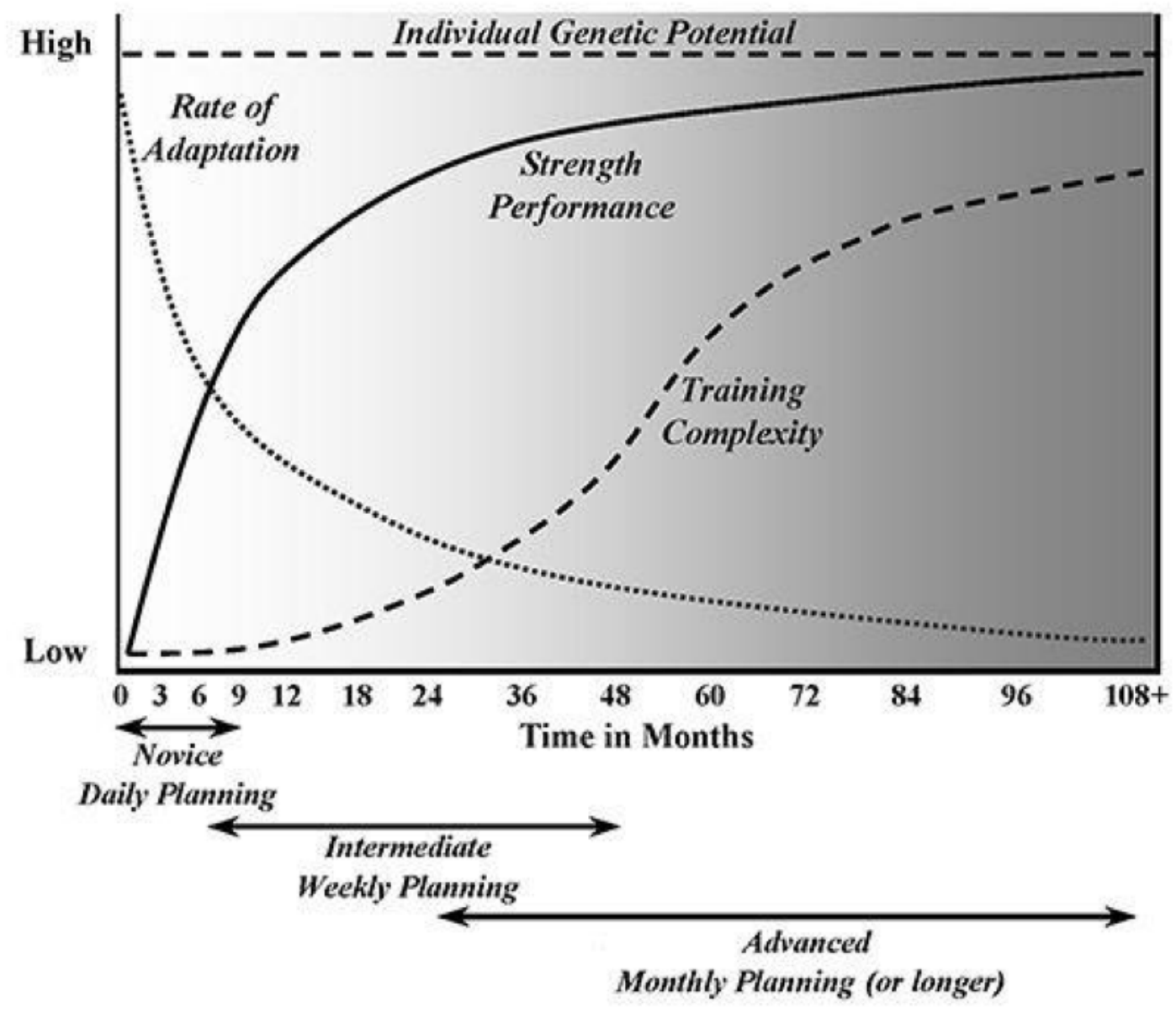

Figure 2.2: Training complexity over time [90]

participate in mobility and corrective exercises that prepare the trainee for safely performing barbell weight lifting movements [106].

Barbell weight lifting movements are often described as "primal" movements [38] because they emulate basic movements that humans have had to perform for millennia in order to survive. As the human race has become more sedentary in the last few hundred years out of convenience, more and more people are underusing the basic movement abilities that their ancestors dutifully passed on to them. This under use, best captured by the common statistic that he average adult sits 8 to 12 hours a day [18], causes movement deficiencies due to 
the atrophying and stiffening of muscles. Properly and consistently practicing moving in these primal movement patterns is to fully express what the human body is capable of, as Socrates alludes to in Figure 2.3. Rippetoe, a couple of thousand years later, poetically describes properly performed barbell weight lifting exercises as, "essentially the functional expression of human skeletal and muscular anatomy under a load" [90]. In order to express the how the human body moves, a trainee must first re-learn how to move their body with proper technique.

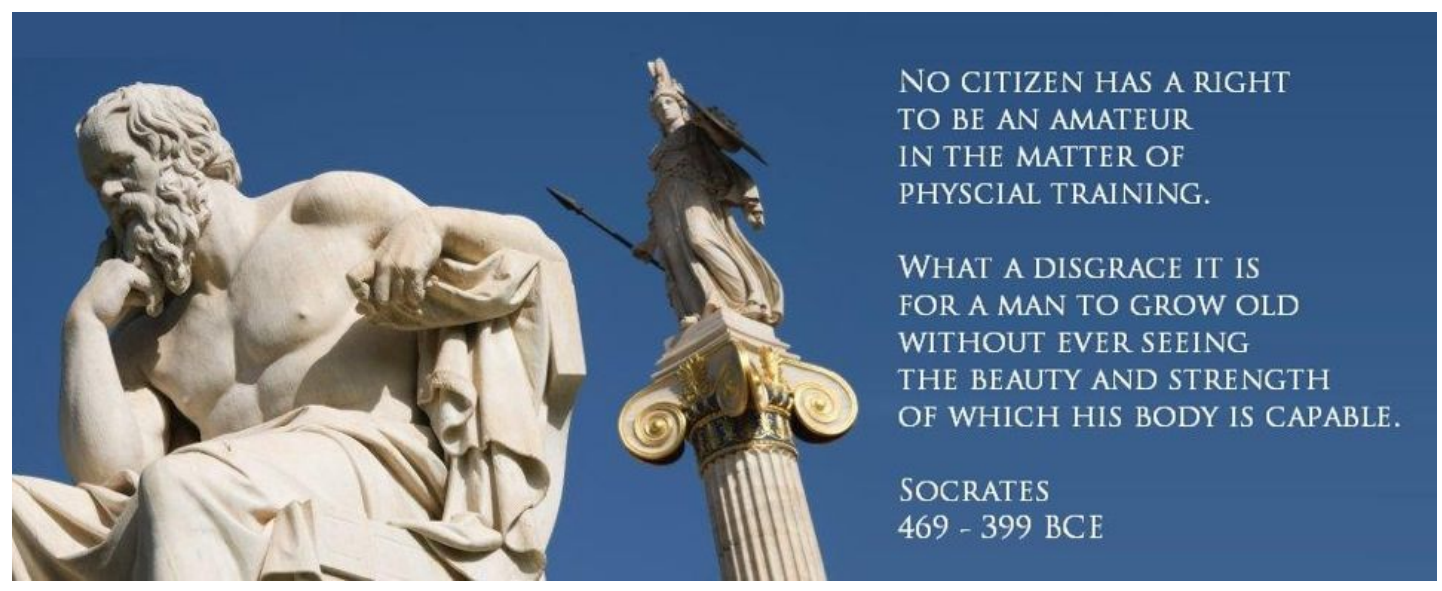

Figure 2.3: Socrates' perspective on fitness [50]

\subsection{Weight lifting technique}

To get a better understanding of how to automate barbell weight lifting technique feedback, we will need to be familiar with what correct and incorrect technique look like and the associated coaching cues for each exercise. The following section describes in detail the correct and incorrect ways to perform the particular barbell weight lifting techniques used in this study: the back squat, bench press, and strict overhead press. 
Naively, from studying physics or even from just lifting anything heavy, the most efficient path for a barbell to take when being lifted, minimizing the amount of work done to the barbell, is a straight, vertical line that is parallel to the force of gravity. This naive assumption holds true with the overhead press, where the trainee must "move their body around the bar" to achieve the optimal bar path [90]. This assumption is invalid for other lifts when studying optimal squat and bench press biomechanics: a small amount of horizontal movement is typically necessary to perform these exercises safely [90]. This small horizontal movement should be subtle and controlled enough by the trainee to be distinguished from a major bar path technique error.

For the standing lifts, the back squat and overhead press, the trainee should always attempt to keep the weight centered over the middle of their foot as seen in Figure 2.4. This is significant because they can best maintain their balance when the load is vertically over that point [90]. Any movement that causes the load to move forward or backward of the mid foot causes an unnecessary moment arm that results in extra work for the trainee and introduces the potential for injury for trying to overcompensate for the mistake [90].

The following sections describe the correct and incorrect ways to perform the specific lifts studied by the researchers. The correct and incorrect techniques were compiled by consulting Starting Strength [90] and working with Cameron Van Wye, a Certified Strength and Conditioning Specialist who works in the Cal Poly Athletic weight room as a Strength and Conditioning coach. 


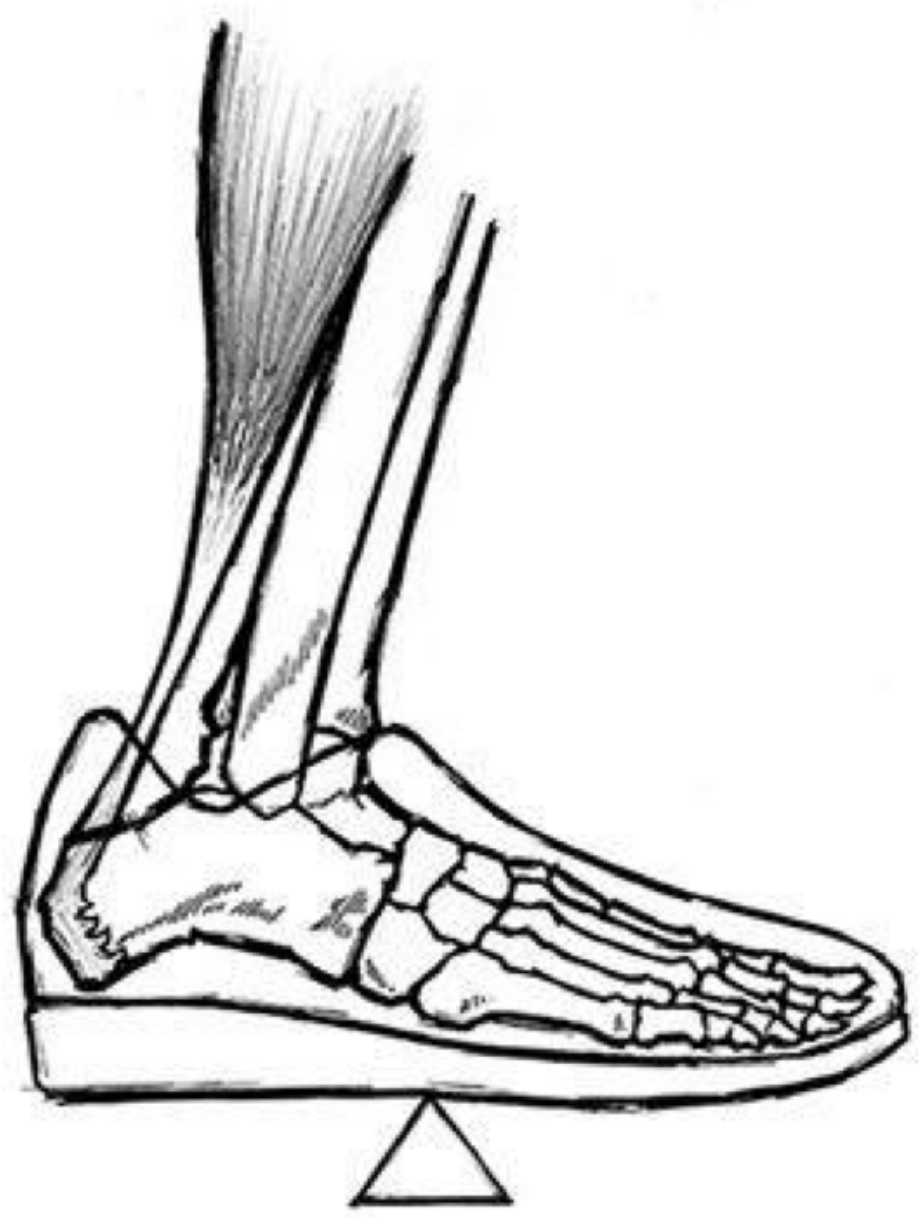

Figure 2.4: How the body is balanced during standing barbell exercises [90]

\subsubsection{Back Squat}

The back squat is often called the "King of Exercises" because it arguably trains nearly all of the muscles in the body when using a significant enough load [90]. The back squat is an incredible athletic tool when performed correctly as seen in Figure 2.5, but could be potentially dangerous if the trainee does not strictly adhere to correct technique. 


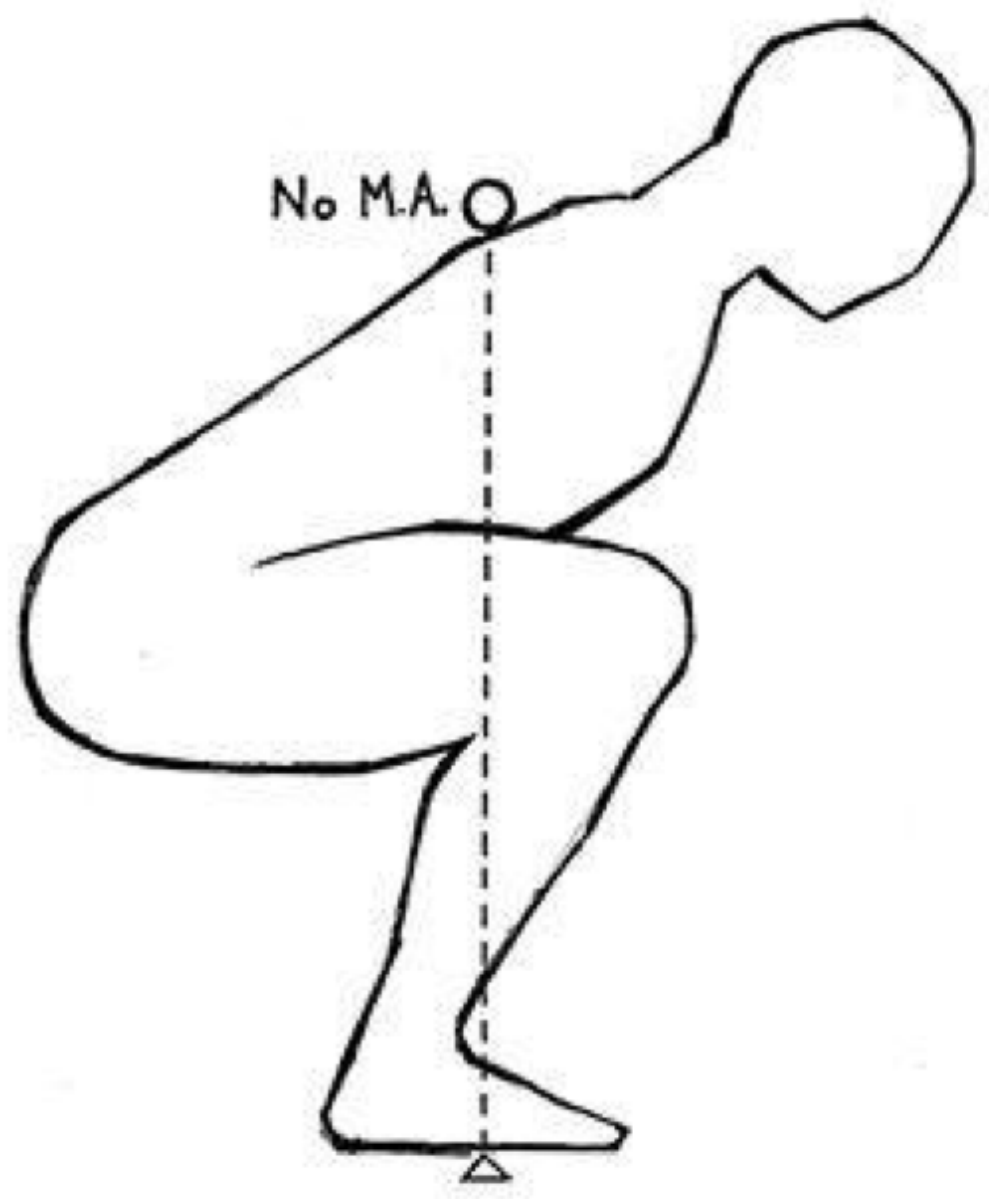

Figure 2.5: The correct way to squat [90]

\subsubsection{Correct technique}

1. Use thumbless grip wider than shoulder width

2. Tighten back by pulling elbows up and place the bar on the "shelf" that Trapezius muscles create

3. Keep head neutral with spine and tuck chin as if to hold a tennis ball between chin and chest

4. Feet are shoulder width apart

5. Toes are pointed out at a 30 degree angle 
6. Unrack bar in same position as squatting

7. Knees track over the toes

8. Perform the Valsalva maneuver by breathing in and bracing core with a tight weight belt if possible which helps create more internal pressure to stabilize the trunk

9. When crease of hip is lower than the knee, visualize pushing up with lower back to activate hamstrings

10. Be mindful of the "master cue", which is to try to track the bar over the middle of the foot as seen in 2.5

\subsubsection{Incorrect technique}

The technique deficiencies recorded and labeled for the back squat were:

\section{Head not looking forward}

The head position of the trainee is critical for setting the spine for proper positioning under a load. A common adage in weight lifting is "where you look is where your body will go". One may take away "don't look down, look up" from that advice when performing the squat. This is not strictly correct because too much looking up can, at the very least be disorienting for the trainee, at worst, unsafe according to Rippetoe, due to "placing the cervical spine in extreme over extension and then placing a heavy weight on the trapezius muscles directly underneath it" [90]. Unfortunately, looking up is how squatting is taught in high school weight rooms all across the country [90]. Improper head positioning may prevent proper hip drive from happening by not allowing the trainee's spine to be neutral throughout the lift as well as loss of balance due to not having a close reference point to look at [90], so the sensor may pick this up as a forward or backward translation 
in the X-Z plane.

2. Upper back rounding

The upper back position of the trainee is critical for spine stability and therefore safety. The trainee's upper back and chest may not be flexible enough to stay in thoracic extension, so the trainee may need to do mobility exercises to correct this muscular imbalance. The upper back should be held in a neutral position for the duration of the lift. This technique deficiency could be characterized by a falling pitch and/or forward translation of the sensor in the $\mathrm{X}-\mathrm{Z}$ plane due to the shoulders coming forward and down during the lift.

3. Lower back rounding

Lower back rounding most likely will occur at the bottom of the squat due to the inflexibility of the trainee's hamstrings. The hamstrings are stretched to their maximum length above a parallel squatting position, so the trainee will attempt to get to parallel by allowing their pelvis to posteriorly tilt and their lower back to round. Rounding the back increases the shear stress on the vertebrae of the lower back and makes them more prone to injuries. Much like upper back rounding, having the lower back staying in a neutral position is critical for safety and successfully completing the lift. This is because the lower back is what connects the power-producing hips and legs to the load-bearing portion of the body. Keeping the lower back rigid helps facilitate the force produced by the hip and leg drive to be applied to the load across the shoulders. The trainee can perform various hamstring stretches and mobility exercises to practice getting to parallel without rounding their lower back. This technique deficiency could be characterized by a falling pitch and/or forward translation of the sensor in 
the $\mathrm{X}-\mathrm{Z}$ plane.

4. Back overextended / too vertical

Trainees can get the mental model of performing a back squat like a front squat. The front squat is performed with a much more upright torso, which is appropriate for where the load is for that exercise, because that is how the trainee can keep the load over the middle of the foot. which is the point where the trainee can maintain the best balance. Having a vertical torso for the back squat is not appropriate, however, because the load is on the back of the trainee. If the torso is too vertical, then the load will be behind the mid-foot line and the trainee will be off balance. This technique deficiency could be characterized by a rising pitch and/or backward translation of the sensor in the X-Z plane.

5. Hips come too far out / chase with back

At the bottom of the squat, instead of pushing up against an "invisible weight" with the lower back as Rippetoe suggests [90], the trainee will move their hips back and up instead of forward and up. Moving the hips back greatly reduces the load on the trainee's hamstrings and instead shifts the load to the lower back. This is not optimal because now the hamstrings are not contributing to moving the load and the lower back is put into a potentially injurious situation. The trainee should concentrate on coming directly up, vertically translating their hips and torso at the same time, out of the squat as they are lowering themselves down. This technique deficiency could be characterized by a lowering pitch and/or forward translation of the sensor.

6. Hips roll under spine at top of repetition

As a trainee reaches the top of a squat, they mistakenly tilt their pelvis 
posteriorly to get a sense of a hip thrust. Performing a hip thrust at the top of a squat is unnecessary and tilting their pelvis in this fashion is much like rounding their lower back at the bottom of the squat; posteriorly tilting the pelvis increases the shear stress experienced by the lower back vertebrae and increases the likelihood of injury. This technique deficiency could be characterized by a lowering pitch and/or forward translation of the sensor.

7. Did not reach parallel

The trainee's hip crease does not pass the top of their knee, so they are not experiencing the full range of motion of the exercise, which is optimal for increasing athletic performance as well as safety. The trainee should work on flexibility and mobility if they are unable to reach the parallel squatting position. This technique deficiency could be characterized by a jerky, abrupt change in the linear acceleration signal from the sensor at the bottom of the squat.

8. Did not stand up all the way

The trainee does not fully stand up out of the squat, but instead starts to go back down when they are most of the way up. The trainee does not experience the full range of motion for each repetition and are missing out on the athletic and safety benefits of performing the full range of motion for this exercise. This technique deficiency could be characterized by a jerky, abrupt change in the linear acceleration signal from the sensor at the top of the squat caused by the ends of the bar flexing.

9. Knees not spread out

The trainee's knees do not track over their toes. Most likely, their knees cave in to provide a "better" support structure for standing up out of a squat. The lateral movement under a heavy load can increase the risk of injury 
for the several knee ligaments and cartilage. This technique deficiency could be characterized by a grinding type of repetition resulting in a linear acceleration signal from the sensor that is closer to zero than the average signal.

10. Heels coming off of the ground

Heels coming off of the ground is indicative that the trainee is out of balance and the load is no longer over the mid-foot where they can achieve the best balance. A way to solve this is to have the trainee curl their toes in their shoes to force them to center their weight on the middle of their foot. This technique deficiency could be characterized by a lowering pitch and/or forward translation of the sensor due to the trainee becoming out of balance and creating more work.

11. Wrists roll forward or backwards during lift

The trainee may want to adjust the bar position during a lift because their initial set up was off. This extra movement wastes energy and potentially could move the bar out of balance from the middle of the foot, creating more work for the trainee to do. This technique deficiency could be characterized by a changing pitch of the sensor.

12. Did not complete repetition

The trainee had to be spotted or otherwise did not complete this recorded repetition and will not be included in the technique training set.

\subsubsection{Bench Press}

The Bench Press is one of the most common exercises performed for strength gain. A trainee's bench press is often the only metric other trainees ask about to gauge their overall strength. Many trainees do not perform the bench press 
optimally and could be lifting more with the correct technique, as seen in Figure 2.6 .

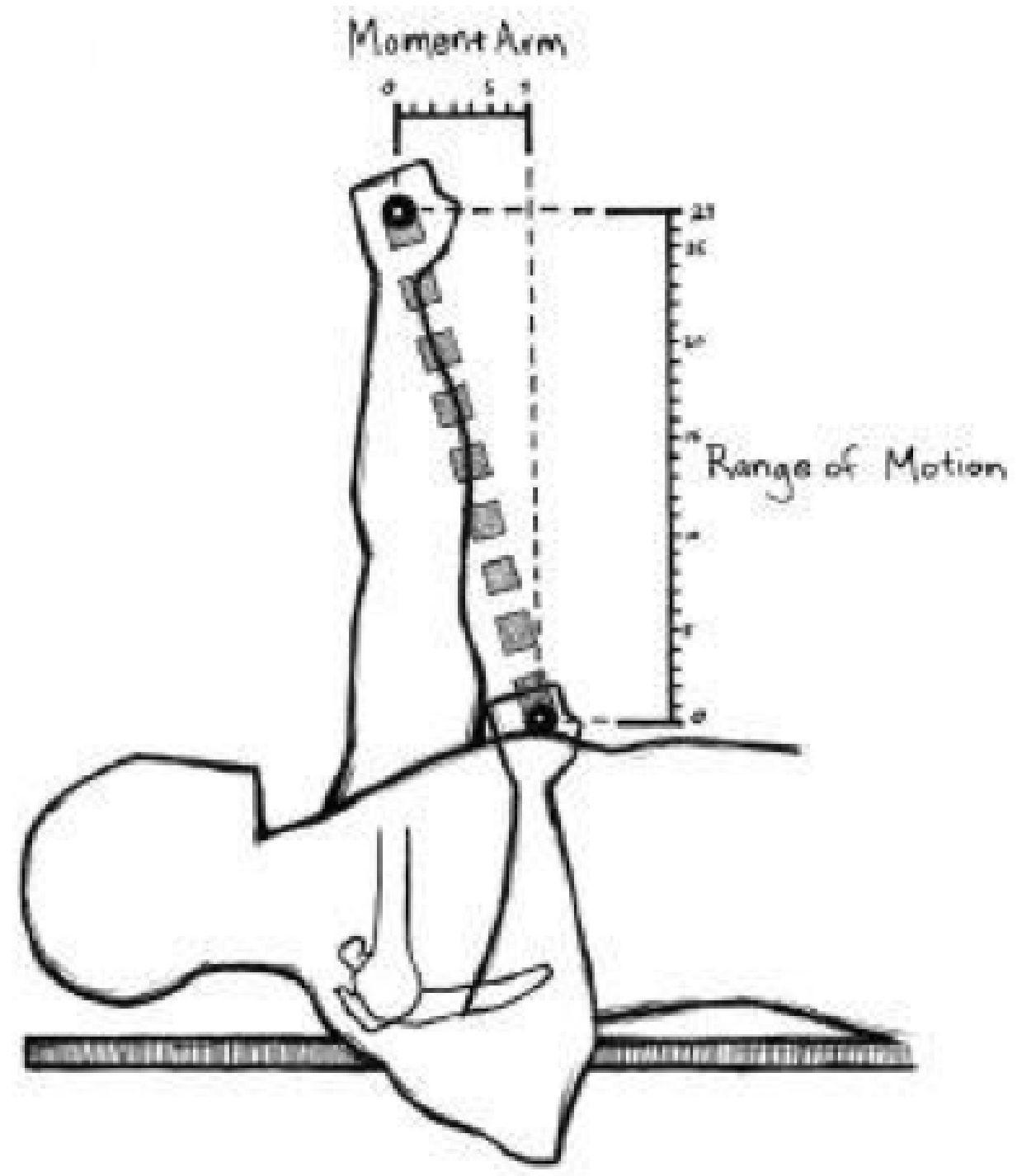

Figure 2.6: The correct way to bench press [90]

\subsubsection{Correct technique}

1. Contract upper back to give stable points of contact on bench

2. Grip the bar with a thumb over grips about shoulder width apart 
3. "Break the bar". Contract lats to keep elbows at approximately 45 degrees.

4. Firmly plant feet on ground and use legs to "drive" power during set to upper body. Do not change foot position during set

5. Engage glutes to protect lower back from over extension

6. Inhale and exhale at the top of the repetition. Holding air in while performing repetition increases stability.

\subsubsection{Incorrect technique}

The technique deficiencies recorded and labeled for bench press were:

1. Upper back not tight

The upper back is not contracted and is not providing the trainee increased stability on the bench.

2. Elbows out

The trainee is not engaging their lats, therefore not pulling their elbows in to a safer angle. Shoulder impingement is more likely with elbows closer to 90 degrees away from trunk, as seen on the right side of Figure 2.7 Bar path efficiency is compromised for safer forces on the shoulder joint [90]. This technique deficiency could be characterized by minimal translation in the $\mathrm{X}-\mathrm{Z}$ plane.

3. Glutes not engaged

The trainee is not using their glutes to help facilitate transfer of leg drive to the upper body and are not protecting their lower back vertebrate from shear stress caused by over extension. The glutes also give a solid point of stability on the bench. This technique deficiency could be characterized by instability in the orientation of the bar.

4. Excessive lower back arch 
The trainee is arching lower back such that their glutes are no longer on the bench. This is a dangerous position for the lower back because it applies a larger than average shear stress to the vertebrae.

5. Bounce off of chest

The trainee is not performing the repetition in a controlled manner. The bounce helps the trainee bench more weight, but does not necessarily facilitate strength gains. This could be detected by LIFT as a significantly higher maximum acceleration than other repetitions.

6. Does not touch chest

The trainee does not lower bar to chest to achieve full range of motion at the bottom of the repetition. Full range of motion is important for getting the most work out of a repetition. This could be detected by LIFT as an absence of noise from when the athlete would have touched their chest or a significantly lower maximum acceleration for the feature set as a whole.

7. Does not lock out

The trainee does not go through full range of motion at the top of the exercise. The triceps are not being contracted as much as they could be if they were paused at the top of the bench press. This could be detected by LIFT as a sudden change in acceleration as the weights usually clink at the top of these type of repetitions.

8. Wrists roll forward or backwards during lift

The trainee is wasting energy by rolling their wrists because they did not set up properly. This could be detected by LIFT due to the change in orientation of the bar.

9. Left/right side comes up before other

Trainee is either laying crooked on bench press or they have a muscular 
imbalance that should be corrected. This could be detected by LIFT due to the change in orientation of the bar.

10. Jerky repetition

The repetition is very uncontrolled in general. The trainee may be under trained or is trying to push the bar up as fast as they can. This could be detected by LIFT due to the high variability in linear acceleration and orientation.

\section{Did not complete repetition}

The trainee had to be spotted or otherwise did not complete this recorded repetition and will not be included in LIFT analysis.

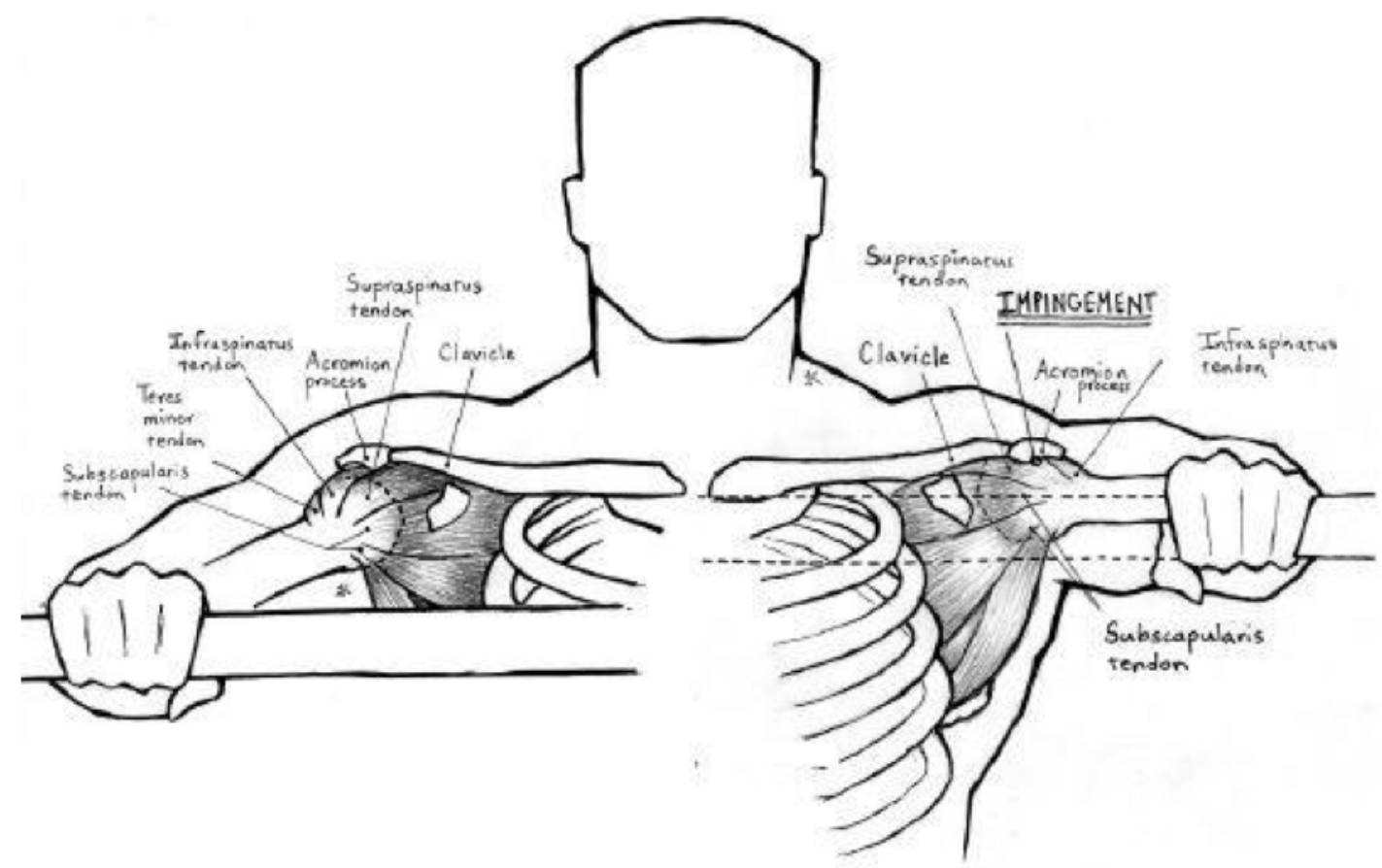

Figure 2.7: Anatomy of a bench press with elbows at 45 degrees on the left and 90 degrees on the right. [90] 


\subsubsection{Overhead Press}

The overhead press hearkens back to intuitively what a person would do with a barbell right after they picked it up off of the ground. As Rippetoe says "When you press a barbell overhead, you celebrate the spirit of weight training" [90].

Overhead press is a very useful lift to complement bench press in building upper body pushing strength. This is because a properly performed overhead press, as seen in Figure 2.8, can help develop a stronger bench press by developing stronger shoulder and tricep muscles, but the other way around is not necessarily true $[90]$.

\subsubsection{Correct technique}

1. Grips on bar should be shoulder width apart

2. Elbows and chest should be up and upper back contracted to support the bar

3. Elbows should stay in and not flare out to maximize movement efficiency and reduce risk of shoulder injury

4. Bar path should pass right in front of face, as soon as bar clears head, contract glutes and move torso under bar to keep the bar balanced over mid foot.

\subsubsection{Incorrect technique}

The technique deficiencies recorded and labeled for strict overhead press were:

1. Chest/Shoulders/Elbows down

The trainee's upper body can get fatigued and start to round forward with 

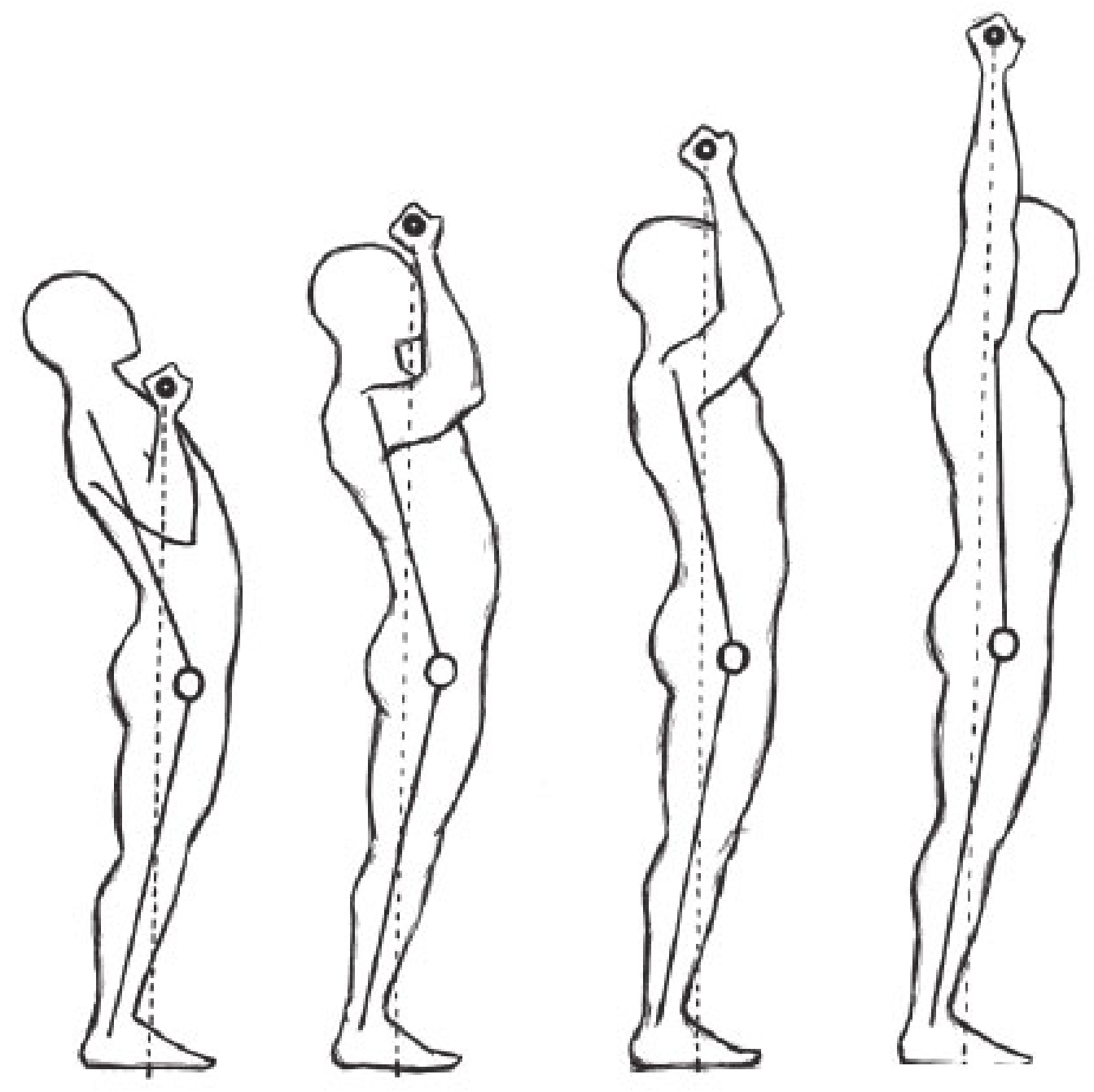

Figure 2.8: The correct way to overhead press [90]

the weight. This can cause inefficient bar paths and increased risk of injury due to the shoulder not being stabilized by the upper back.

2. Didn't use hips

The trainee did not use their glutes to get under the bar as they pressed it up.

3. Pushed bar away / Started too far out

The trainee did not use an efficient bar path by first pushing the bar out 
instead of straight up. This path inefficiency can also occur when the trainee start with the bar too far away from their body. These errors cause more overall work because it moves the bar out of balance with the mid foot, as seen in Figure 2.11.

4. Didn't get under bar

The trainee did not move their torso forward under the bar to help keep the bar over the middle of the foot as seen in Figure 2.9.

5. Too much layback

The trainee leaned back too much in the process of pressing the bar. This movement puts unecessary shear stress on the lower back vertebrate and can increase the potential for injury.

6. Wrists roll forward or backwards during lift

The trainee wastes energy rolling their wrists as they move through a lift as seen in Figure 2.10. Moving the wrists under a load could increase risk of injury as well.

7. Left/right side goes up before the other

The trainee either started in an unbalanced grip position or they have a muscular imbalance that needs to be corrected.

8. Jerky, uncontrolled rep

The trainee performed generally uncontrolled repetitions that are potentially dangerous to their shoulder health.

9. Elbows out

The trainee flared their elbows out, resulting in an inefficient bar path and subjecting their shoulders to potentially injurious positions.

10. Did not complete repetition

The trainee had to be spotted or otherwise did not complete this recorded 
repetition and will not be included in LIFT analysis.

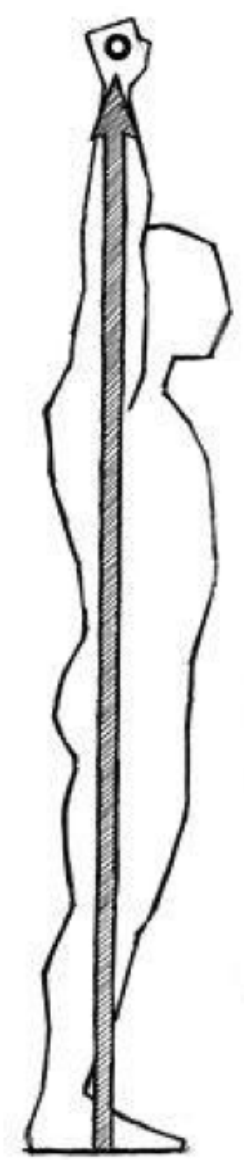

Figure 2.9: The importance of getting under the bar during the press to keep it balanced over the mid foot [90]

\subsection{Weight lifting technique feedback}

Athletes need constant feedback to correct their weight lifting movements to ensure that they are safely lifting weights and can continue to make progress. Traditional forms of obtaining feedback have been limited to taking video of the lift and self-critiquing or having a trainer critique it, or listening to a training partner or a trainer's advice immediately after a lift. A new field of study is emerging 

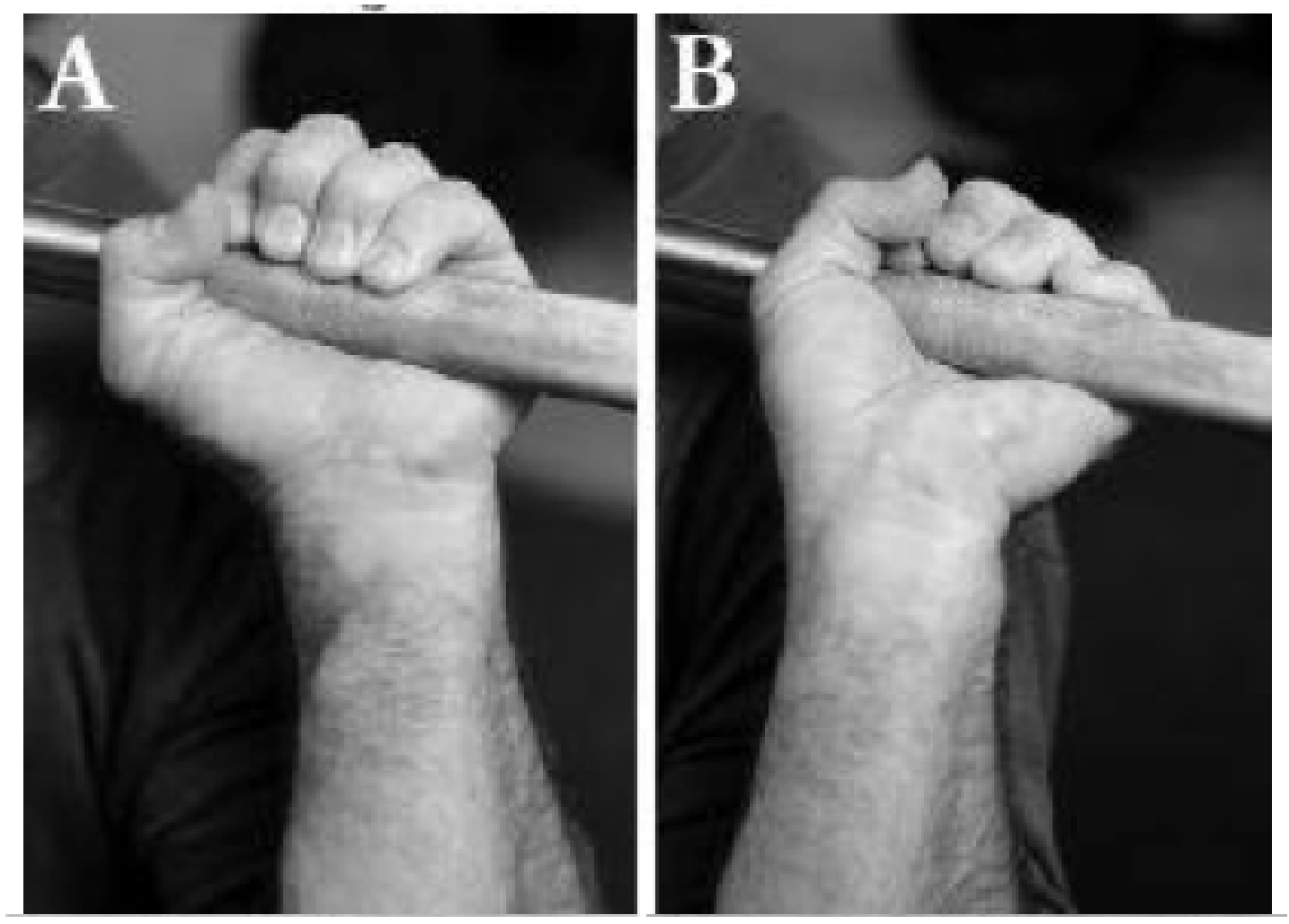

Figure 2.10: Keeping wrists straight to avoid wasting energy [90]

to attempt to give objective feedback to weight lifters by using technology in non-invasive ways.

\subsubsection{Visual feedback}

Tracking the bar path of a lift with a camera has been the primary form of visual feedback for weight lifters since the 1970's. Garhammer's biomechanical analysis of olympic lifts could be considered the birth of video bar path analysis. In Garhammer's work, "Biomechanical Profiles of Olympic Weightlifters", he took video of the 1984 Olympic Weightlifting competition and analyzed the bar path using various digitizing systems and an Apple IIe computer [45]. A more modern approach to video bar path analysis can be seen in Figure 2.12. Video feedback is 


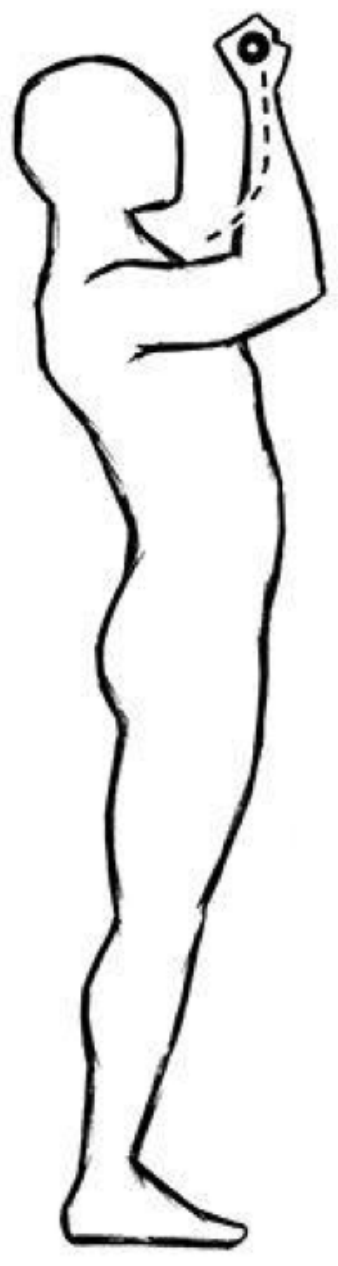

Figure 2.11: Using an inefficient bar path for the press [90]

not limited to those with high speed cameras anymore. Desktop applications such as Kinovea [9] provide coaches and athletes with a toolkit that can help them analyze not only weight lifting video, but also other sports specific activities. Mobile applications such as Barsense [4], Coach's Eye [6], and Platform Helper [14] now make bar path tracking and other sports specific analysis accessible for anyone who has a mobile device. These applications do not have any inherent knowledge about the "correct technique" for any given activity, so they rely on the expertise of coaches and athletes who are using the application to know what 
they are looking for in terms of correct technique. Recording stable video on a mobile device by setting up a tripod or other arrangement is not always possible, so there is a need for devices to measure parameters about sport specific activity.

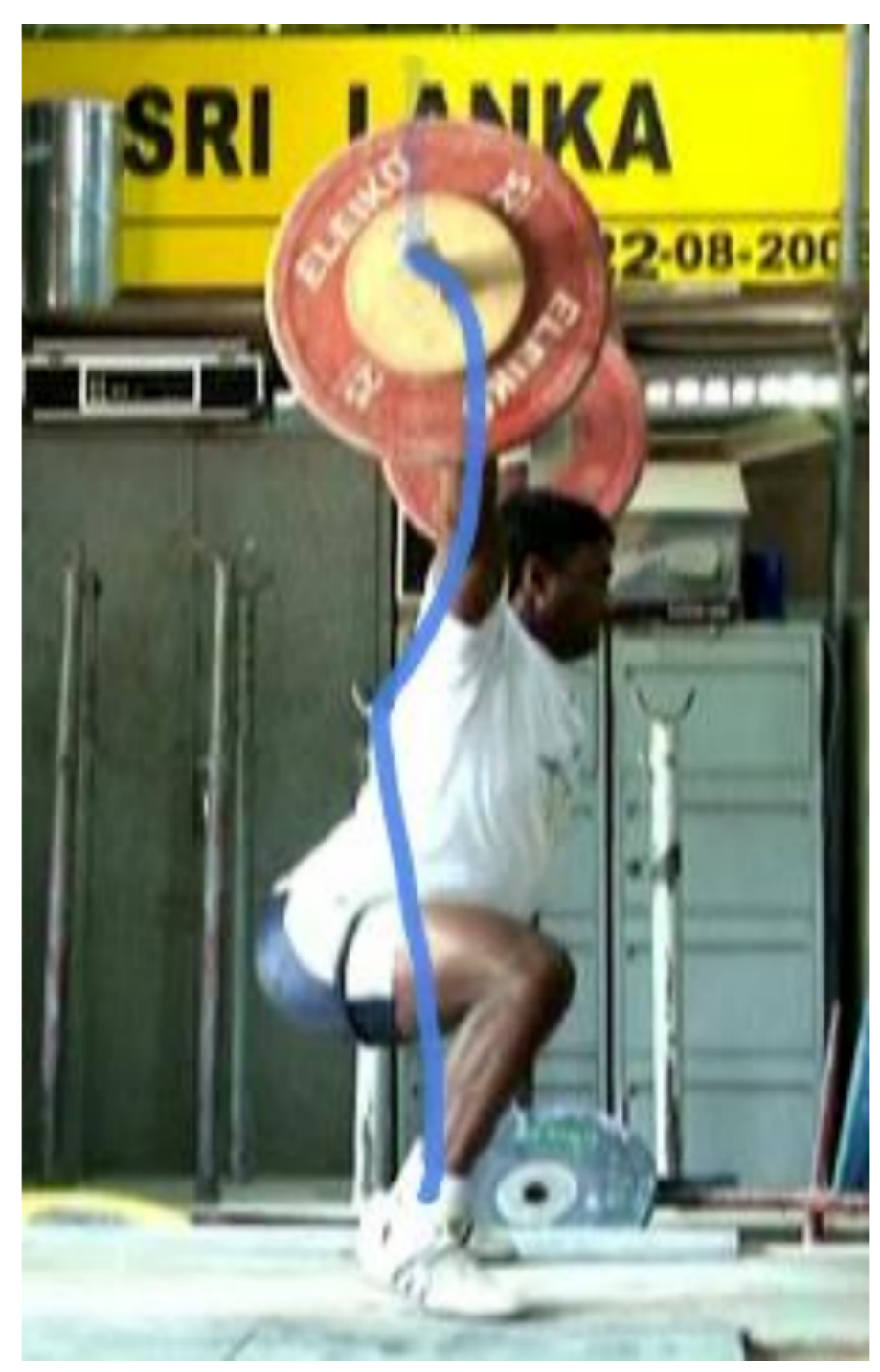

Figure 2.12: Visual bar path feedback [61]

\subsubsection{Wearable devices}

There has been a recent increase in interest in integrating technology with fitness to help better quantify the amount and quality of the exercise a person does. 
The reason for this spike in interest is twofold. First, motion-tracking sensors (accelerometers, gyroscopes, etc) have become smaller and cheaper [108, 112], thus more viable for mass commercial production and adoption in many devices. This new generation of sensors is able to be integrated into devices such as smart phones [92], wristbands [1, 5, 7,8,13,20], arm bands [16], clothing [2], and running shoes [12]. The second reason interest in fitness technology has increased is because logging, attractively presenting, and holding users accountable with data can help motivate a user to continue with an exercise program that will help lead to long-term health [57]. Figure 2.13 shows a snapshot of the wearable device market.

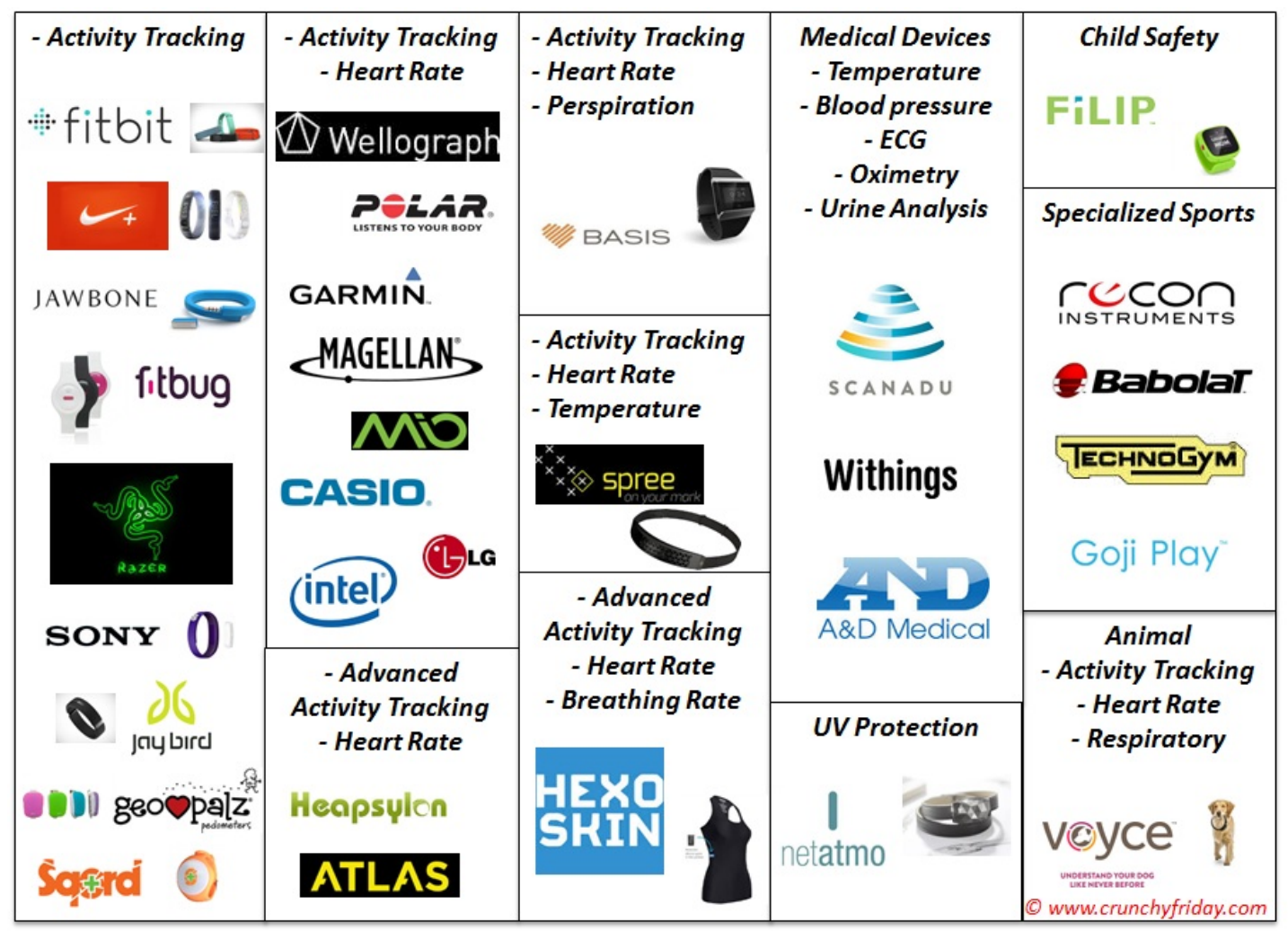

Figure 2.13: A snapshot of the wearable device market [60]

The first generation of such fitness-tracking devices largely consisted of "glorified pedometers" [80] worn as a wristband or armband, such as Fitbit [7], Jawbone 
Up [8], and Nike+ [13]. These devices were largely limited to currently estimating how many calories a trainee is burning, and distance walked or ran. The second generation fitness devices introduced by the incumbents as well as new competitors, such as Amiigo [1], Basis [5], Polar Flow [15] and Withings [20] started to add in new features, such as sleep tracking and heart rate monitoring to up the ante in the increasingly competitive health and fitness device market. In late 2013 to early 2014, several next-generation fitness trackers were announced in what can only be described as a tour de force in one-upmanship or complete coincidence. Athos [2], Atlas [3], Moov [11], and PUSH [16] were announced within weeks of each other. On the surface, each device will attempt to track an athlete's performance in terms of exercise-specific metrics, such as how many repetitions and sets were completed as well how much force the athlete produced. The seemingly more advanced Atlas and Moov also claim to be able to do technique analysis on exercises, which can decrease an athlete's risk of injury $[3,11]$. All of these next-generation fitness devices, as seen in Figure 2.14, are crowd-funded business ventures that, as of this writing, have not shipped to customers yet. The main differentiator between these devices seems to be form factor (armband vs. wristband, etc) and the algorithms that power them. The features claimed by these consumer-level devices far exceed features offered by current academic researchers. It is suspected that due to the lucrative opportunities by taking a fitness device to market, a would-be researcher is understandably hesitant to divulge valuable intellectual property in an academic research paper. The LIFT analysis system intends to become an open-source complement to these proprietary fitness devices.

One way LIFT can leverage integration with future fitness tracking devices such as the Moov is that it would benefit from improved sensors and software 

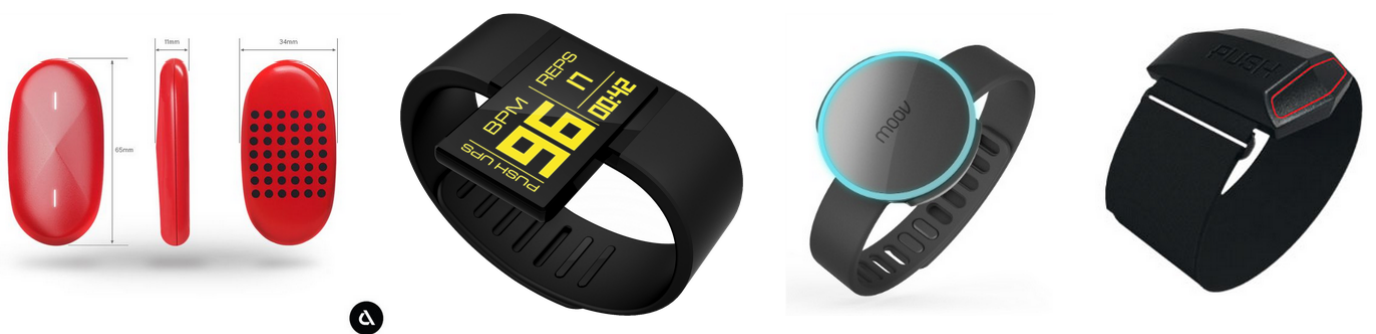

Figure 2.14: Training feedback device competitors $[2,3,11,16]$

API support. The Moov is built specifically for sport technique feedback, so the hardware design takes this into account, whereas the YEI IMU currently used by LIFT is just a general-purpose IMU. The Moov API could include native support for position, velocity, and repetition tracking which would be much more useful application-specific information than general acceleration, gyroscope, and magnetometer data. The Moov has demonstrated an intriguing level of position tracking already via a developer demo where the Moov seemingly is able to display the $3 \mathrm{D}$ modeled position of punches thrown in real time [10]. This powerful position determination would be able to plug into LIFT as soon as the SDK becomes available. The only concern would be its performance around metallic objects, as seen in the first version of LIFT and discussed in Section 9.6.2. 


\section{CHAPTER 3}

\section{Technical Background}

This chapter will go into detail about the basic inner workings of how mobile devices record and analyze data.

\subsection{How device sensors work}

There are three basic sensors that are used in mobile devices everywhere today: the accelerometer, gyroscope, and magnetometer. These sensors generate data for mobile devices to process and interpret their own movement through space. The next sections will describe what each sensor measures and how they conceptually do so.

\subsubsection{Accelerometer}

The accelerometer measures the acceleration felt by the device over time. Harking back to basic physics, acceleration is the derivative of velocity over time as seen in Figure 3.1.1. Velocity, in turn, is the integral of acceleration over time (as seen in Figure 3.1.1) and the derivative of displacement over time (as seen in Figure 3.1.1. In other words: acceleration contains information about the change in velocity over time for the device.

Acceleration is defined as: 


$$
\vec{a}=\frac{d \vec{v}}{d t}
$$

Velocity is defined as:

$$
\vec{v}=\int \vec{a} d t
$$

Displacement is defined as:

$$
\vec{d}=\int \vec{v} d t
$$

Weight lifting movements, almost by definition by virtue of being performed by humans (as opposed to machines) will have a non-constant velocity, which means that an accelerometer will measure a non-zero acceleration (it wouldn't measure exactly 0 for reasons explained soon).

The main reason why this non-zero acceleration data is useful to measure is because of the Newton's Third Law of Motion, where "to every action there is always opposed an equal reaction" [85]. This law shows that as the trainee moves the mass of the bar through space, they are providing a normal, or opposing, force against the force of gravity. Reviewing Newton's Second Law of Motion in Figure 3.1.1 to define "force":

$$
F=m a
$$

where $\mathrm{F}$ is force, $\mathrm{m}$ is mass, and a is acceleration.

What is interesting about accelerometers however is that both the trainee's applied normal force's acceleration (also called linear acceleration) vector and the normal force due to gravity's acceleration vector are measured [93]. 


$$
\vec{F}=\overrightarrow{F_{\text {applied }}}+\overrightarrow{F_{\text {normalGravity }}}
$$

The reason for the accelerometer measuring the normal force due to gravity requires a deeper look into how accelerometers are modeled.

Accelerometers measure the forces felt much like a mass on springs in a box as seen in Figure 3.1. The sensor if left resting on a surface, will measure an upwards normal force of $1 \mathrm{~g}$ ( $\mathrm{g}$ is the acceleration due to gravity, $9.8 \mathrm{~m} / \mathrm{s}^{2}$ ) because the model mass is being supported by the normal force supplied by the box to equally oppose gravity and causes the mass to stay still as seen in the first case in Figure 3.1. In free fall, the accelerometer will measure 0g because both the mass and box with the attached springs will be accelerating at the same rate, so no difference will be measured as in the second case in Figure 3.1. The common accelerometer measurement will have both the normal force due to gravity as well as any movement forces applied to the sensor combined into the same output acceleration vector as seen in the fourth case of Figure 3.1.

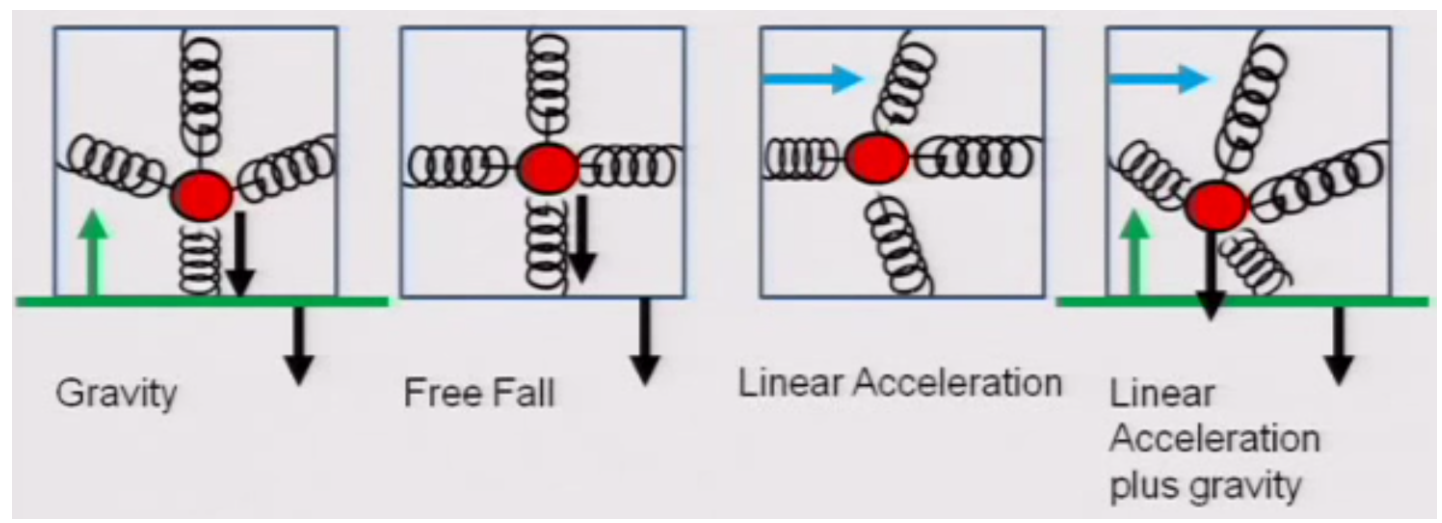

Figure 3.1: A model of how an accelerometer measures acceleration [93]

Therefore, to obtain the linear acceleration vector, the normal force due to gravity vector must be subtracted from the accelerometer's output acceleration 
vector. This is a non-trivial task, because the device must have a very good idea of what orientation is with respect to the Earth, or else this could introduce large amounts of error as seen in Table 3.1. We will cover a solution that the researchers use to do this later in Section 3.2.

\begin{tabular}{|c|c|c|c|c|c|c|}
\hline 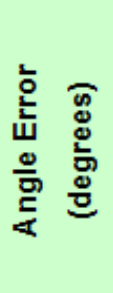 & 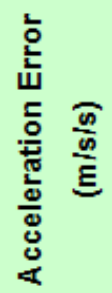 & 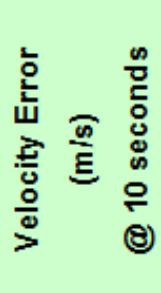 & 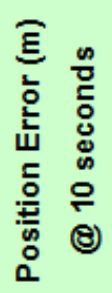 & 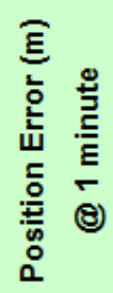 & 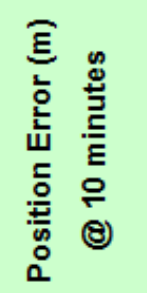 & 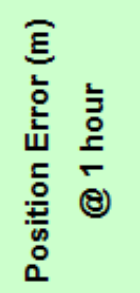 \\
\hline 0.1 & 1.71 & 0.2 & 1.7 & 62 & 6157 & $221.6 \mathrm{E} 3$ \\
\hline 0.5 & 8.55 & 0.9 & 8.6 & 308 & $30.8 \mathrm{E} 3$ & $1.1 \mathrm{E} 6$ \\
\hline 1.0 & 17.11 & 1.7 & 17.1 & 615 & $61.6 \mathrm{E} 3$ & $2.2 \mathrm{E} 6$ \\
\hline 1.5 & 25.66 & 2.6 & 25.6 & 924 & $92.4 \mathrm{E} 3$ & $3.3 \mathrm{E} 6$ \\
\hline 2.0 & 34.22 & 3.4 & 34.2 & 1232 & $123.2 \mathrm{E} 3$ & $4.4 \mathrm{E} 6$ \\
\hline
\end{tabular}

Table 3.1: How quickly error can accrue over time due to incorrect initial orientation calculations $[26]$

A weakness of the accelerometer is that it is a very noise-prone sensor [93]. It can be very accurate to measure patterns over the long term, but there can be very wide range of minima and maxima even within a single second as seen in the green signal in Figure 3.5. This noisiness will be compensated for in Section 3.2 .

\subsubsection{Gyroscope}

The gyroscope measures the angular velocity felt by the device over time [93]. It has a very quick, accurate short-term response as opposed to accelerometers, but is prone to long term drift inaccuracies [93]. The gyroscope is able to measure angular velocity by using a constantly rotating platform with a oscillating mass as 
seen in Figure 3.2 [46]. The rotating platform in conjunction with the oscillating mass is used to measure the Coriolis effect [46]. The Coriolis effect is the principle that objects that are rotating on the same plane about the same point will not have the same angular velocity. More precisely, the object on further out on the platform will experience a larger angular velocity than the object closer to the center of the platform. How much faster the mass is moving when it gets to the outer edge of the circular platform as opposed to when it is on the inner side of the platform is measured and taken into consideration when the gyroscope is measuring angular velocity.
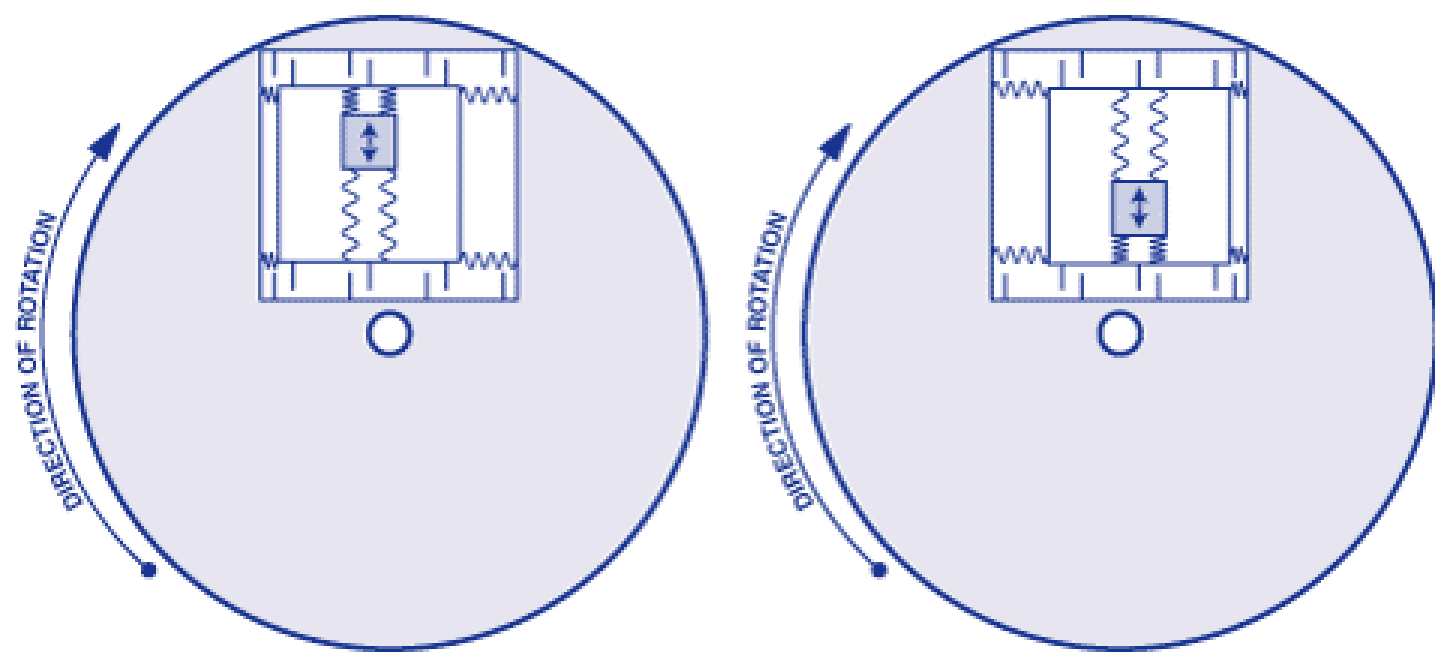

Figure 3.2: A model of how a gyroscope measures angular velocity [46]

Angular velocity is a useful measurement for the device to record when tracking barbell movement through space because as the trainee moves the bar through space, they could rotate the bar about an axis, which would cause the trainee to perform more work on the bar than necessary [90]. This rotation could lead to not just an inefficient bar path, but could cause the trainee to move the bar out of balance with their body and risk injury. 


\subsubsection{Magnetometer}

The magnetometer measures the strength and direction of the surrounding magnetic field over time. The magnetic field detected by a magnetometer can either represent the Earth's magnetic field or the resultant magnetic field interactions with ferrous metals or other sources of electromagnetic activity in the magnetometer's environment. A basic, non-MEMS magnetometer measures the magnetic field by the Hall effect, which works by "detecting a voltage across a metallic surface in response to a magnetic field that is perpendicular to the metallic surface" [35], as seen in Figure 3.3. The voltage that is seen across the metallic surface is called the Hall voltage, and can be used to infer how strong the magnetic field is in a given direction. A naive way to make a compass that measures the magnetic field in three dimensions would be to align three magnetometers all perpendicular to each other and turn the resultant vector of Hall voltages into a magnetic field magnitudes. Newer MEMS-based magnetometers use the Lorentz force and other more advanced methods to determine the magnetic field [107], but all that is needed to be gathered from this background is the intuition that the Earth's magnetic field is always in play and can be measured by electronic equipment, hence the term "electromagnetic" field.

The main weakness of the magnetometer in this application is that it can be influenced by localized ferrous metals as seen in Figure 3.4. This is a problem that will affect LIFT as it is close to ferrous metals such as barbells and plates, but can be remedied by putting space between LIFT's sensor and any surrounding ferrous metals. 


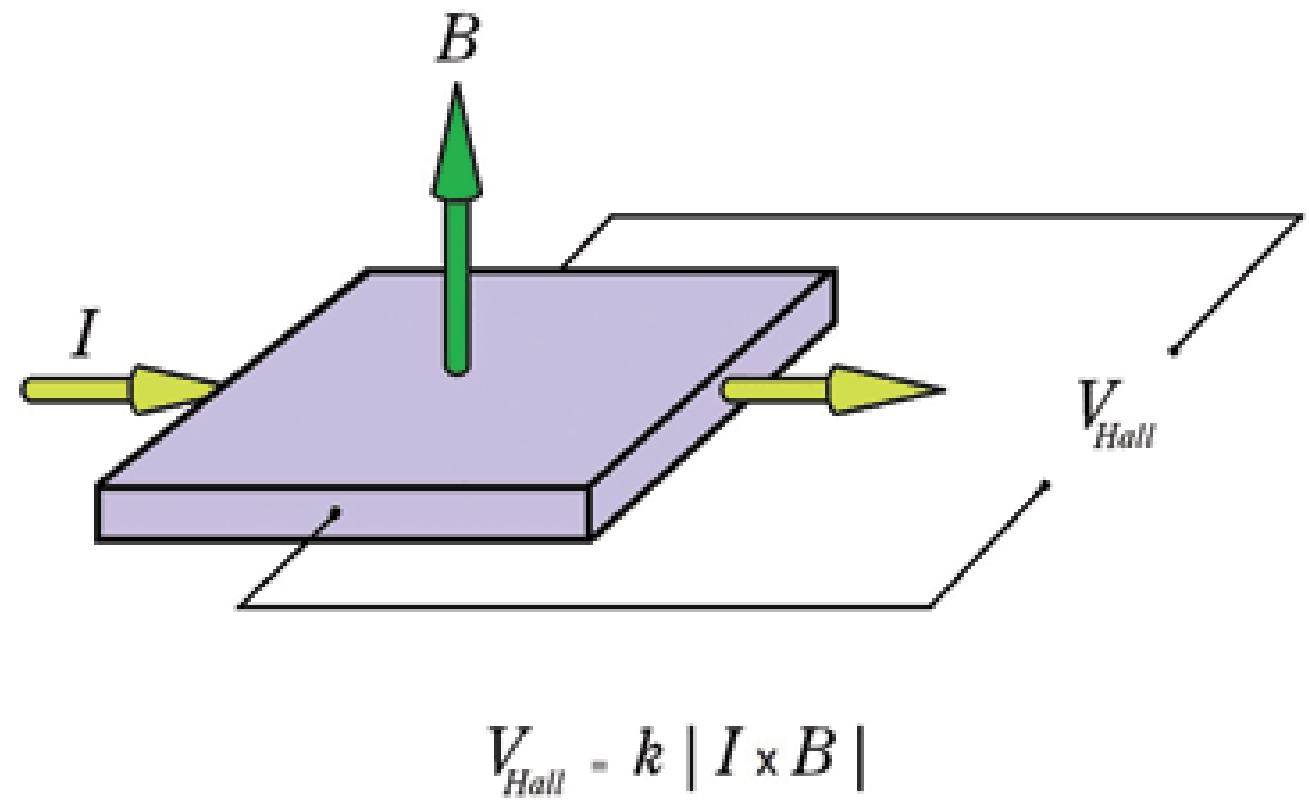

Figure 3.3: A model of how a magnetometer measures the magnetic field [35]

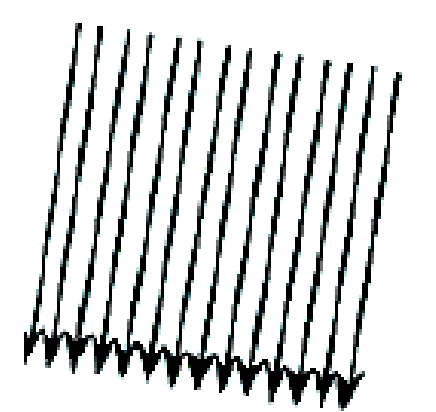

Earth's mannetic neld In absence of metal vehicle

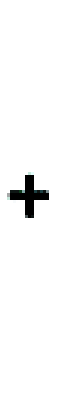

Wagnetic dipole produced by Terrous materlals

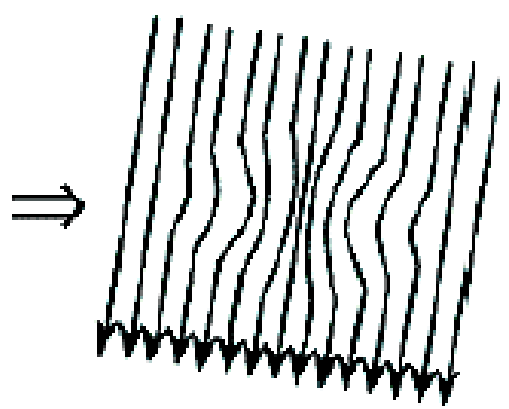

Resultant magnetic anomaly in Earth's magnetic neld

Figure 3.4: An example of how the magnetic field can be altered by a ferrous metal [25]

\subsection{Combining sensor data together}

The sensors described in the previous sections all have weaknesses that prevent any one sensor from perfectly modeling its intended measurement, but the re- 
sults from all three of these sensors can be combined together in such a way to compensate for each sensor's weakness and produce more accurate data values to be analyzed.

An online real-time Kalman filter is used on the YEI 3-Space Bluetooth Sensor to accomplish this goal of mutual sensor compensation [21]. The Kalman filter is a linear estimator, which means that its output is made from a linear system of equations and best models systems that have linear behavior [24]. The Kalman filter also assumes that any noise in the system has a gaussian distribution [24].

The filter works by first making a prediction of the future state of the sensor, $\overline{{\operatorname{lin} A c c_{t}}}$, by taking a prior state, $a c c_{t-1}$, and adding any compensatory terms to it, such as gyroscope data, gyro ${ }_{t}$, when determining the orientation of the device [24]. These terms are multiplied respectively by appropriate governing equations and weights, A and B, for each type of sensor [24]. Noise cannot be completely avoided when reading from the gyroscope, so there is a noise term $\varepsilon[24]$.

$$
\overline{\operatorname{linAcc}_{t}}=A * a c c_{t-1}+B * \text { gyro }_{t}+\varepsilon
$$

Next, Kalman filter makes a prediction for the measurement of the sensor $\left(\overline{a c c_{t}}\right)$ by passing a sensor prediction function $(f(x))$ the $\overline{\operatorname{linAcc_{t}}}$ term and accounting for error $\varepsilon$ that is possible [24].

$$
\overline{a c c_{t}}=f\left(\overline{\operatorname{lin} A c c_{t}}\right)+\varepsilon
$$

Finally, the Kalman filter determines compensated estimate for the given sensor, $\operatorname{lin} A c c_{e s t}$, by measuring the actual sensor in question, $a c c_{t}$, subtracting 
it from its predicted value, $\overline{a c c_{t}}$, and multiplying that by the pre-tuned Kalman gain vector, $k$. This quantity is then added to the $\overline{\operatorname{lin} A c c_{t}}$ term.

$$
\operatorname{lin} A c c_{e s t}=\overline{l i n A c c_{t}}+k *\left(a c c_{t}-\overline{a c c_{t}}\right)
$$

The scenarios for how this equation play out in a couple of ways. One way is that the predicted measurement, $\overline{a c c_{t}}$, could be the same as the measurement,

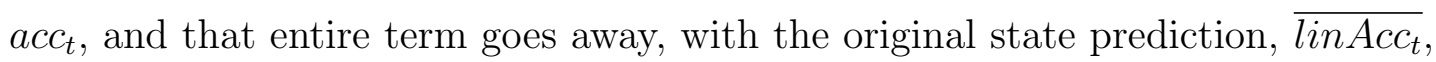

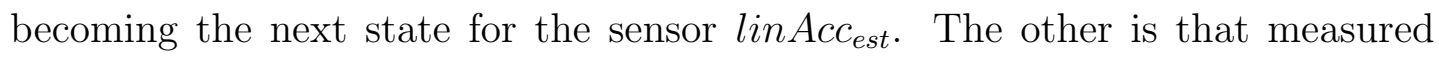
sensor value, $a c c_{t}$, could be different from the predicted value $\overline{a c c_{t}}$, so the Kalman gain vector $k$ can scale how much that difference affects the estimated value $\operatorname{linAcc}_{\text {est }}$. This general process is expanded to three dimensions in the LIFT system due to unrestricted freedom of movement on the barbell.

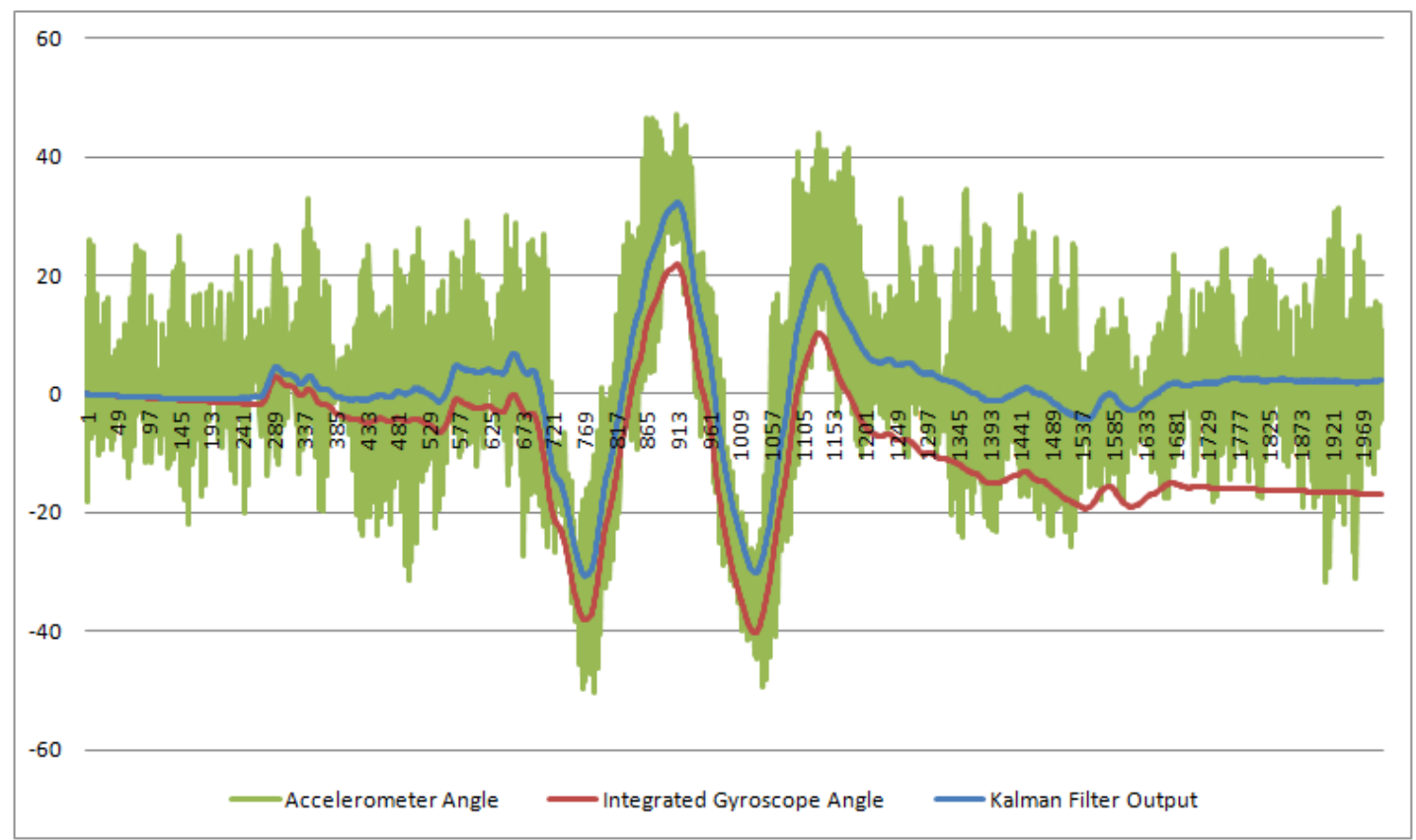

Figure 3.5: An example of the output of a Kalman filter given accelerometer and gyroscope signals [27] 
In Figure 3.5, the green signal represents example raw accelerometer measurements and the blue one are the cleaner, Kalman filtered measurements.

\subsection{How data classification systems work}

Classification algorithms are a type of "machine learning". Classification algorithms can "learn" to predict the label of given input values when "trained" with a representative data set for a specific application. This fact makes them interesting to apply to domains that have the potential to generate a lot of data that also have known domain-specific labels. Classification algorithms shine where the pattern in the raw data itself is not clear to the human eye, but it can be teased out and differentiated with statistical features that machine learning algorithms act upon.

Statistical features such as mean, variance, standard deviation, max, min, etc. can be used to describe a particular instance of an input signal. These sets of statistical features are, naturally, called feature sets. Specific instances of feature sets can be paired with application-specific labels that can tell a classification algorithm that those set of features are an example of what a given label "looks" like. Classification algorithms can take these feature-set label-pairings and build their own model of how the underlying data defines those specific labels and can be used to predict future, unlabeled data sets.

How classification algorithms build their internal model predicting feature sets is algorithm-dependent. The following sections will discuss how the particular classification algorithms used by LIFT work internally and their suitability for LIFT's specific data set. 


\subsubsection{Decision tree}

A decision tree is a graph of nodes with feature checks that can classify unlabeled feature sets. Quinlan et al. describes an example decision tree in pseudocode:

\section{if wage increase first year $\leq 2.5$ then \\ if working hours $\leq 36$ then class good \\ else if working hours $>36$ then \\ if contribution to health plan is none then class bad \\ else if contribution to health plan is half then class good \\ else if contribution to health plan is full then class bad \\ else if wage increase first year $>2.5$ then \\ if statutory holidays $>10$ then class good \\ else if statutory holidays $\leq 10$ then \\ if wage increase first year $\leq 4$ then class bad \\ else if wage increase first year $>4$ then class good}

Figure 3.6: Verbal example of a decision tree [89]

Figure 3.6 reads not unlike a series of nested "if-statements" found in virtually every programming language. The difference with these "if-statements", however, is that these were automatically generated by a data set that had a number of feature sets and corresponding labels. This ability for a computer to "learn" what feature values best represent the particular label is the fundamental building block for the LIFT system. Manually defining feature guidelines for how a "good" weight lifting technique should look would be tedious and would not lead to a very robust system because different people have different limb lengths and variations of correct technique. The more optimal way is to get as wide of a variety of body types as possible. This method covers as many cases of different limb lengths and technique variations as possible for the demographics of the testing facilities in use by the researchers. 


\subsubsection{Decision Tree Algorithm}

An adapted version of Quinlan's C4.5 decision tree induction algorithm will be discussed here for clarity [41]:

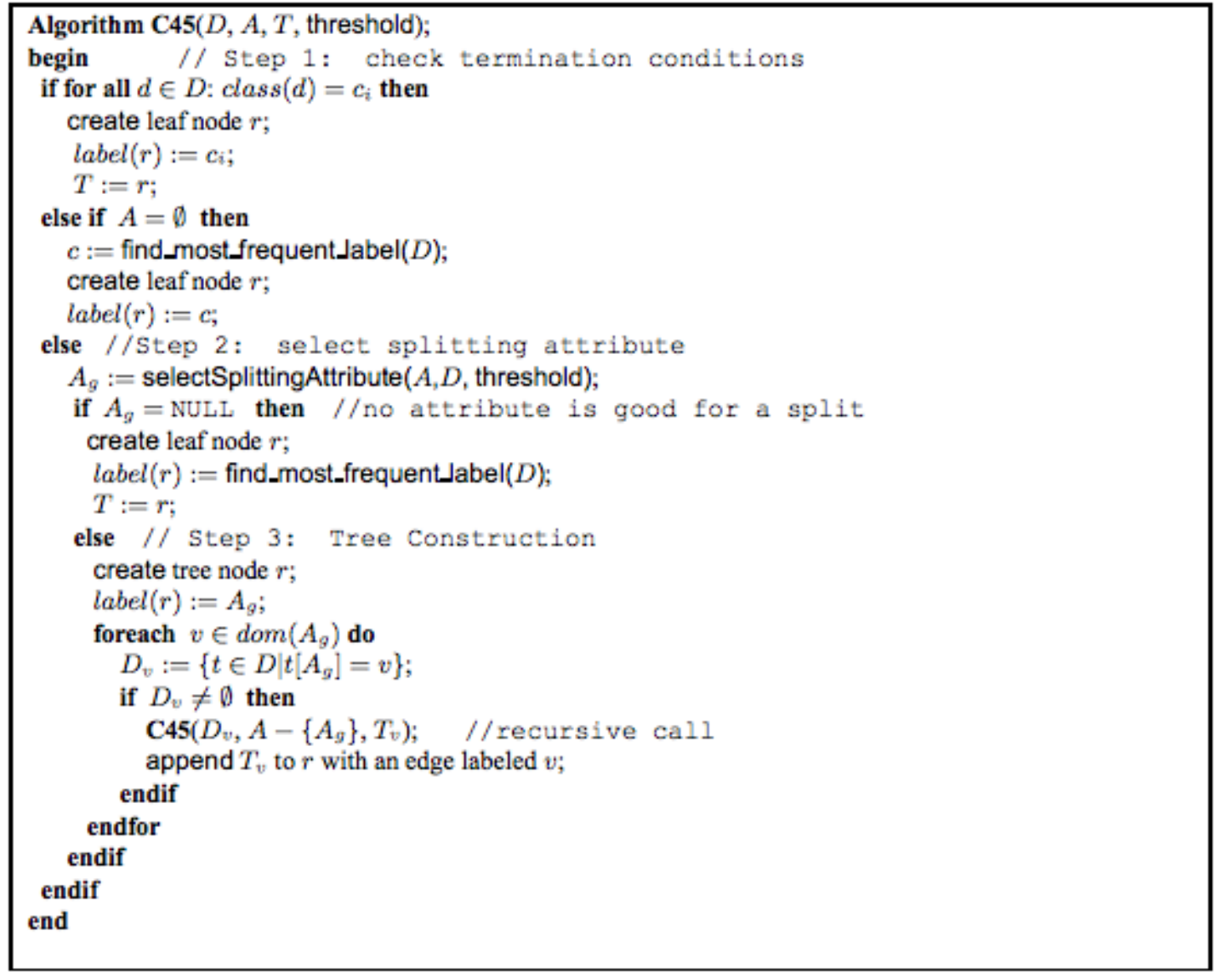

Figure 3.7: C4.5 algorithm for decision tree induction [41]

The C4.5 algorithm as described in Figure 3.7 is a method to help recursively build decision trees based on their features and how much information is gained at each leaf node. The core action at the heart of this algorithm is called "splitting" as seen in Figure 3.8. A splitting node creates two paths in the tree structure to further categorize a given unlabeled feature set. An example of these splitting nodes is seen in Figure 3.9. 


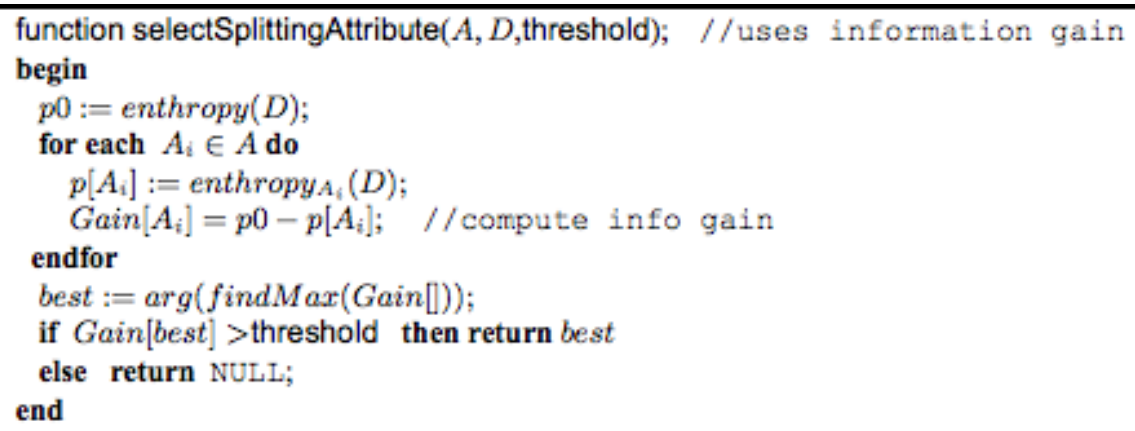

Figure 3.8: selectSplittingAttribute() functions using information gain and information gain ratio measures. [41]

These nodes are created for the highest information gain features in a feature set [41]. Each feature can only be split on once in a given path [41]. This rule ensures that the algorithm will eventually reach the termination conditions and not produce an incredibly large tree that is "overfitted", or created to be too specific for future feature sets. The algorithm's stopping conditions are when the data set contains only instances with the same label or when there are no more attributes to split upon. The current splitting attribute is determined by calculating the potential "information gain" for each feature in the current feature set [41]. The algorithm then uses the feature with the most gain as the splitting feature. The value of the feature to be split upon is determined by the feature values within the domain subset that are currently used in the recursive function. 


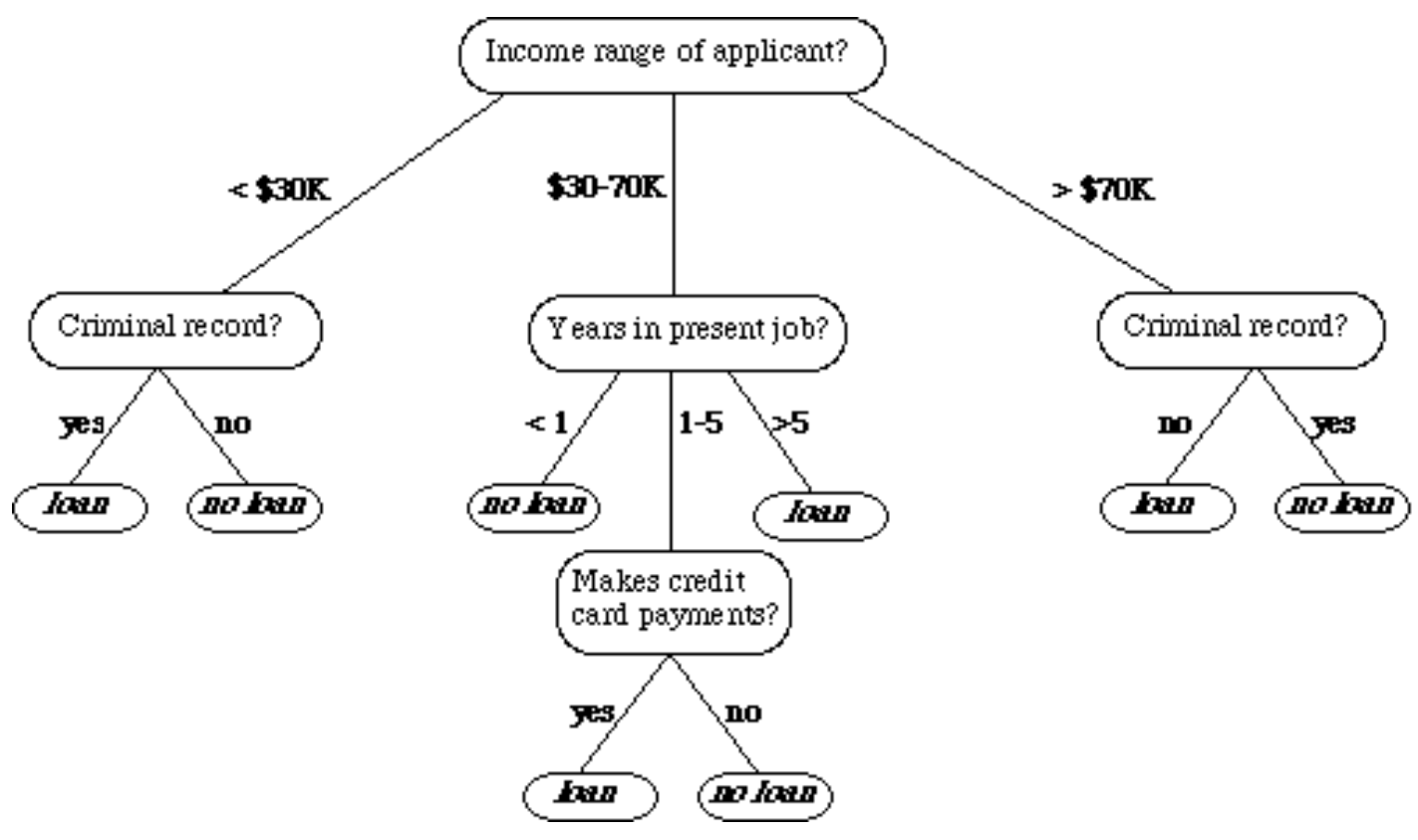

Figure 3.9: A visual example of a decision tree [113]

Once the decision tree is built, it can be used to classify unlabeled feature sets. The measurement of how well the decision tree classifies feature sets is the subject of the next section.

\subsubsection{Classifier Evaluation}

A constructed classifier is typically tested against its own data set to ensure that it is at least proficient enough at classifying feature sets that it has "seen" already before moving on to attempt to classify unlabeled feature sets. Accuracy is defined as the number of correctly labeled feature sets out of the total number of feature sets in the data set [41].

$$
\operatorname{accuracy}(\text { label })=\frac{T P}{T P+F P+F N+T N}
$$

Precision is defined as the number of true positive results from classification 
over the sum of both true and false positive results from the classifier [41].

$$
\operatorname{precision}(\text { label })=\frac{T P}{T P+F P}
$$

Recall is defined as the number of true positive results over the sum of true positive and false negative results from the classifier [41]. In other words: accuracy reflects how the classifier performed overall at labeling feature sets, precision reflects how well the classifier performs in labeling feature sets correctly, and recall reflects how well the classifier labels all of the particular features sets of a particular label.

$$
\operatorname{recall}(\text { label })=\frac{T P}{T P+F N}
$$

\subsubsection{Random forest}

Random forests have been studied to be empirically well-performing ensemble classification algorithms, but are hard to formally prove certain properties of Random forests about outside of their definitions [55].

Random forests were first introduced by Breiman [33] as an ensemble method of classification. An ensemble classifier combines multiple classifiers together to achieve a better result than any one classifier could do. For Random forest classification, the algorithm builds a user-defined number of decision trees that are based on a given data set [33].

The pseudocode in Figure 3.10, which is discussed in [65], demonstrates how a random forest is trained and queried. The "forest" is a list of trained decision trees that all get queried when the forest gets an unlabeled feature set. Each decision 


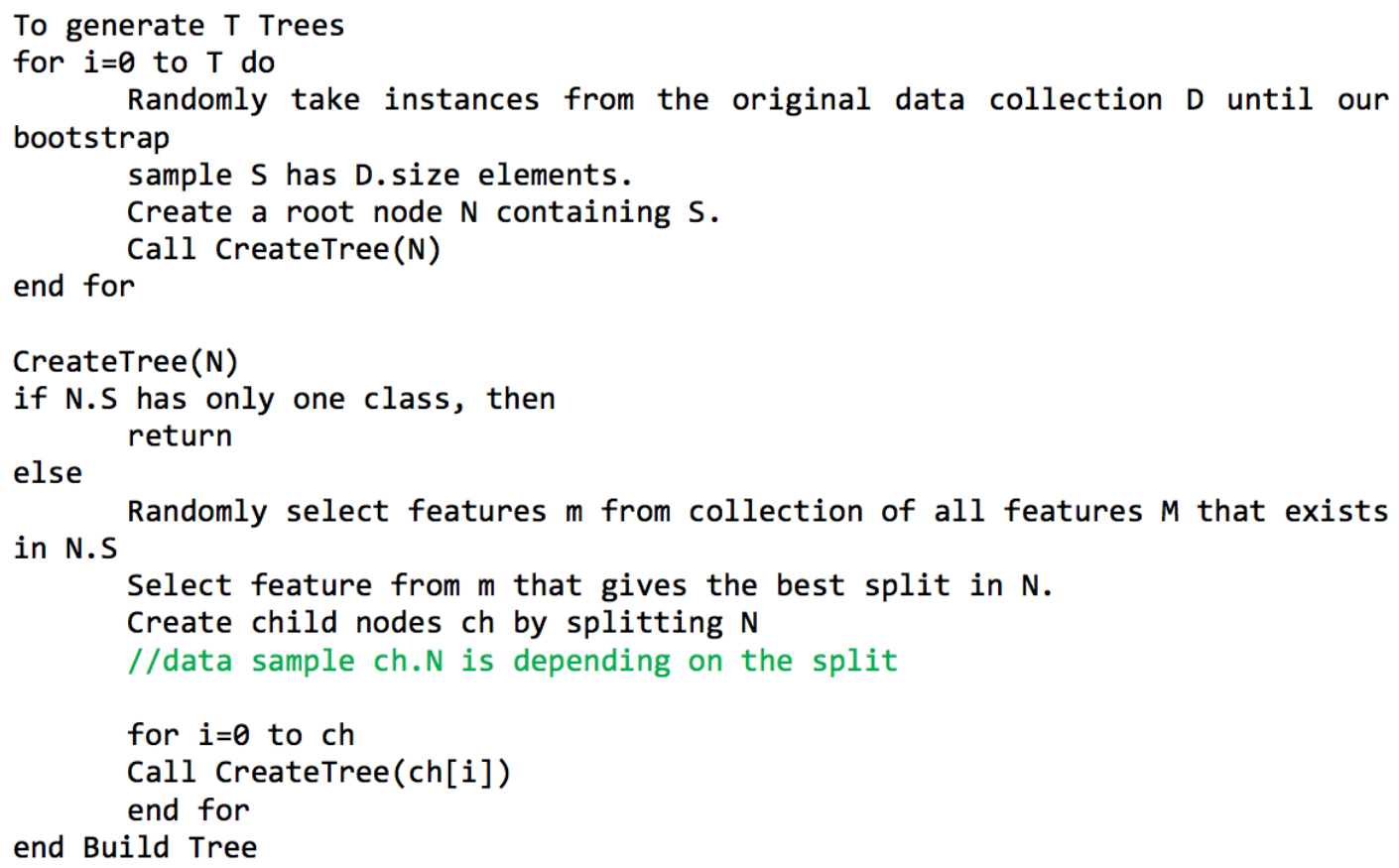

Figure 3.10: Random Forest Pseudocode [65]

tree is trained by selecting random features from a subset of the data set that is uniformly chosen with replacement (called "bagging" or "bootstrapping") [23]. A simple method for evaluating the label of an unknown feature set is to have each decision tree simply vote for the label it thinks the feature set represents [23]. When considering individual decision trees, their evaluation of what the label should be is highly varied, but since it is a forest that is built by uniformly selecting sample feature sets from the data set, there should be a label that is closely correlated to the underlying function or model, if it exists [23].

\subsubsection{Extra-tress}

The Extra-trees splitting algorithm was proposed by Geurts et al. in a 2006 paper [47]. The algorithm was designed to potentially “... build totally randomized trees whose structures are independent of the output values of the learning sample" 
[47]. The algorithm also breaks from the pack by learning from the entire data set, instead of a uniform sample like the Random forest algorithm [47] Geurts et al. described the algorithm as the following:

\section{Split_a_node $(S)$}

Input: the local learning subset $S$ corresponding to the node we want to split

Output: a split $\left[a<a_{c}\right]$ or nothing

- If Stop_split( $S$ ) is TRUE then return nothing.

- Otherwise select $K$ attributes $\left\{a_{1}, \ldots, a_{K}\right\}$ among all non constant (in $S$ ) candidate attributes;

- Draw $K$ splits $\left\{s_{1}, \ldots, s_{K}\right\}$, where $s_{i}=$ Pick_a_random_split $\left(S, a_{i}\right), \forall i=1, \ldots, K$;

- Return a split $s_{*}$ such that $\operatorname{Score}\left(s_{*}, S\right)=\max _{i=1, \ldots, K} \operatorname{Score}\left(s_{i}, S\right)$.

Pick_a_random_split $(S, a)$

Inputs: a subset $S$ and an attribute $a$

Output: a split

- Let $a_{\max }^{S}$ and $a_{\min }^{S}$ denote the maximal and minimal value of $a$ in $S$;

- Draw a random cut-point $a_{c}$ uniformly in $\left[a_{\min }^{S}, a_{\max }^{S}\right]$;

- Return the split $\left[a<a_{c}\right]$.

\section{Stop_split $(S)$}

Input: a subset $S$

Output: a boolean

- If $|S|<n_{\min }$, then return TRUE;

- If all attributes are constant in $S$, then return TRUE;

- If the output is constant in $S$, then return TRUE;

- Otherwise, return FALSE.

Figure 3.11: Extra-Trees splitting algorithm [47]

The pseudocode in 3.11 enables "randomizing both attribute and cut-point choice while splitting the tree node" [47]. This randomization allows the tree to be built independently of the labels for the classes, which can lead to higher performance in some applications [47]. Geurts et al. found the Extra-trees algorithmic complexity to be $O(n \log (n))$, which is on par with other tree-based classification algorithms, but he claims to have a significant speedup due to the "simplicity of the node-splitting procedure" [47].

Geurts et al. reported the results of an empirical study comparing classifica- 
tion algorithms' performance and accuracy to each other [47]. The Extra-trees algorithm was not only validated to be as accurate as other state-of-the-art classification algorithms, but its performance was competitive as well as long as there is enough memory [47]. The increased randomness comes at a cost: the algorithm produces 1.5-3x more leaves than the random forest algorithm [47]. This should not be an issue as long as the amount of memory dedicated to running Extra-trees scales with the size of the data set. 


\section{CHAPTER 4}

\section{Related Work}

There is a large and ever-growing body of research that investigates the use of sensor and video motion capturing systems to quantify the movement of the human body in order to augment some aspect of it.

\subsection{General motion tracking systems}

Researchers have pushed the envelope of joint configuration and body segment movement tracking by improving upon the sensor filtering methods available, specifically with different types of Kalman filters [64,111]. These Kalman filters often take in multiple IMU inputs, and research has been done about how to most effectively synchronize the data from their readings [36]. LIFT makes use of an Kalman filter that is applied by the IMU device.

There have been many lines of research dedicated to advancing the goals related to achieving practical levels of sensor network contexual awareness and event classification $[29,52,59]$. Some fairly general methods for how body sensor networks operate for the purpose of human movement analysis have been patented $[32,43]$. There are also patents for hand motion tracking with video motion capturing systems $[100,103]$. The ramifications of potential enforcement of these patents in the future for academic and commercial development of generalized body motion tracking remains to be seen. 


\subsection{Systems used to augment healthcare}

One of the more obvious uses of motion detecting sensors is for detecting deficiencies in a person's primary means of movement: walking and using their hands. There has been research done with movement detecting sensors in long term gait tracking, neurological tremors, and other movement disorders $[51,62,98]$ Just like in section 4.1, there are many patents in this area of study $[48,70]$. Multiple patents in this field indicate how valuable this technology is in the eyes of the inventors and other stakeholders in the project.

Researchers can also monitor trunk and posture with motion tracking devices to give feedback to the wearer when they have put themselves in less-than-ideal positions for their health $[74,82]$.

The trunk sensors for posture detection are a mild form of ambulatory monitoring. More serious ambulatory monitoring systems involve detecting senior citizen falls and other emergencies $[53,66,101]$. It is evident that these systems are built with widespread adoption in mind because they integrate into smartphones, which are common devices that has movement sensors. LIFT follows these researchers' lead by designing the system with widespread adoption in mind by using a minimally invasive amount of extra equipment to be able to give feedback to weight lifting technique.

In the rehabilitation domain, Moeller et al. created an Android app that aided in quantifying how well a trainee performed various exercises on a balance board and provide visual feedback on how well they did [76]. Researchers have also used motion tracking sensors in upper limb neurorehabilitation [88]. 


\subsection{Systems used to augment general exercise performance}

Running is one of the most basic forms of exercise, but few people, save for possibly very experienced or competitive runners ever consider their running technique because it develops naturally as a person gains experience running [77]. Researchers have built feedback systems with sensors to help with improving running technique $[69,104,115]$. Bicycling is another fairly basic form of exercise once a person learns how to ride one. Training and getting better at competitive bicycling is a more difficult task and is why researchers have developed many different sensors for bicycling, such as heart rate monitors $[28,58,84]$ and power meters $[31,34,67]$. The heart rate monitor enables the cyclist to help determine how hard their cardiovascular system is working and the power cycle gives feedback about how much power the cyclist is generating with each pedal stroke. These pieces of feedback help the cyclist determine what their next racing move should be when competing, or how they can optimize their training. More recent bicycling research includes the use of a Google Glass bicycle coach application [105]. The bicycle coach application gives feedback about how fast the user is going among other performance metrics [105]. Swimming is another aerobic sport like running and bicycling, but has a much higher learning curve when it comes to sport-specific technique. Naturally, researchers have created sensor-based feedback systems to improve a swimmer's performance $[30,81]$.

One of the more novel sport feedback technologies has come in the form of sensors embedded in baseballs to evaluate the performance of pitches that are thrown [69]. The force applied on a baseball during a pitch as well as direction and magnitude of the rotation of a baseball in flight are very important factors in the overall quality of an intended pitch, so an embedded IMU is well-suited to 
collect this type of data.

An application of the general activity recognition technology discussed in section 4.1 is exercise recognition $[29,99]$. The LIFT system and other qualitative exercise feedback systems like it, could be integrated with general activity recognition systems to automatically give exercise feedback once the exercise in question has been identified by the activity recognition system.

Another interest in the body area sensor network field is estimating energy expenditure during exercise [109]. How much a person gains and loses body weight is determined by the number of calories they eat minus the amount they expend by normal activity and exercise. The number of calories a person eats can be tracked in a fairly straightforward way, so this type of research is working on the other term of the "caloric equation" by exploring ways to obtain a more accurate count of energy expenditure.

The end goal of all these types of sport and exercise-specific of research is the same as the LIFT system: to provide feedback to users in order to increase their domain-specific performance.

\subsection{Systems used to augment weight lifting performance}

One of the oldest methods for evaluating a person's maximal strength has always been performing a one-repetition maximum lift. When lifting maximal weights, a person is pushing their muscular and supporting structures' limits, so injuries are more likely to occur [68]. A safer, more efficient method for many in the future that is being researched is to estimate a person's one-repetition maximum with a sensor attached to the particular exercise in question [91].

Pernek et al. used the sensors built into smartphones to automatically count 
repetitions performed in a set of exercises [87]. A similar line of research is trying to determine the best axis for repetition detection, which minimizes the amount of features and computations that need to occur to provide accurate repetition counts [78].

Nejadiana et al. describes a video-based motion capture system that uses a dynamic programming method to evaluate the mechanical cost of various bar path trajectories for the snatch lift [83].

Sato et al. measured the acceleration felt by a barbell through various Olympic lifts and was able to compare barbell accelerations on Olympic lifts between different workout parameters such as intensity [94,95]. Sato et al. in doing this also validated that data collected by an accelerometer on a barbell can be just as accurate as data obtained from high speed video analysis [96,97]. Another similar line of research validated that accelerometers record reproducible front squat data [37]. Yet another similar line of research placed an IMU on a barbell and weight lifting belt, with force plates and camera to validate the accuracy of the values recorded by the IMUs [49]

Velloso et al. developed an on-body weight lifting tracking system that recorded data from four sensors strategically placed on the body, and obtained feature sets based on the mean, variance, standard deviation, max, min, kurtosis, and skew functions [110]. Those feature sets fed into a Random Forest classifier system [110]. The classifier system that was able to detect specific weight lifting technique deficiencies in a bicep curl with a $78 \%$ success rate with leave-one-out subject validation [110]. Velloso et al. doubts that it is feasible to scale this weight lifting classifier system to detect all mistakes for each exercise one wishes to train on [110]. 
The LIFT analysis system will attempt to ease Velloso et al.'s qualms about scalability by restricting the domain of exercises to train classifiers on to barbell movements that most athletes perform in strength and conditioning programs. 


\title{
CHAPTER 5
}

\author{
Methodology
}

The LIFT analysis system will apply Velloso et al.'s methodology of running classification algorithms over derived features of collected sensor data [110]. The main difference is that the LIFT analysis system will attempt to only use one sensor that is strategically and unobtrusively placed on the weight collar secured on a barbell for specific lifts: back squat, bench press, and strict overhead shoulder press.

\subsection{Barbell Lifts}

These barbell lifts were chosen because they are fundamental movements for any athletic strength training program. Athletes do not always have the luxury of having a qualified coach observing them to correct their technique at a moments notice, so the LIFT analysis system may help provide some basic amount of weight lifting coaching to a wider audience in the future.

These particular movements were also chosen because the bar is secure in the hands of the athlete and the athlete's body is between the bar and the ground during most portions of these lifts. The danger for the bar to be dropped and the sensor potentially damaged by the shock of the loaded bar hitting the ground is much higher with other lifts [96]. 


\subsection{Subject testing}

Another key differentiator in this study of weight lifting technique deficiencies is that the study uses many more subjects of varying strength levels. This gives a more diverse data set in an attempt to improve and validate the broader efficacy of the LIFT system.

\subsubsection{Profile of subjects}

The subjects were limited to trainees that train in either Cal Poly's athletic weight room or recreation center. The gender was controlled for males because while there were many potential female athlete participants in the athletic weight room, there were not many corresponding untrained females who lifted weights in the recreation center, which would result in a lack of variety of technique deficiencies to truly validate the LIFT system.

Although the correct bar path for males and females does not change for any given technique, the fact that males on average have bigger bones, and larger yet less flexible musculature than females can possibly affect the results of classifying technique deficiencies in unforeseen ways. These physiological factors can vary greatly between individuals of the same gender, of course, so there is still room for those factors to have an effect on the end result of classifying technique deficiencies. The researchers are attempting to control significant physiological confounding variables in this study by controlling for the participant's gender. The use of this system on female trainees can possibly be the subject of future research and can be compared to the results of this male study.

There is an inherent bias with the given college-aged population of males, 
so future potential research can study the efficacy of the LIFT system on the teenage and older adult populations.

\subsubsection{Realistic technique deficiencies}

Every set recorded and analyzed by the LIFT system will be with non-trivial weight. Velloso et al. in the name of safety, had their subjects intentionally perform technique deficiencies with a $1.25 \mathrm{~kg}$, or almost a $3 \mathrm{lb}$ dumbbell [110]. This is an admirably safe methodology, and validated that it is possible to have an algorithm differentiate between different simulated technique deficiencies, but it is not a particularly useful result for its intended application. A subject performing technique deficiencies with a weight comparable to a pair of shoes while under very little stress will create very different looking data than a subject performing the same exercise with a challenging, yet safe weight. A primary motivation behind this study is to gather "real world" training data, not just data recorded in experiments performed in a laboratory setting. 


\title{
CHAPTER 6
}

\author{
Implementation
}

The sensor that was used in testing the LIFT analysis system is the YEI 3-Space Bluetooth sensor [21]. This sensor is a 9-degrees-of-freedom inertial measurement unit (IMU) and is described in further detail in Figure 6.1. The sensor communicates with an Android Nexus 7 tablet via Bluetooth. The Android tablet has the LIFT data collection app installed on it. LIFT collects the data recorded by the YEI IMU, stores it locally in a SQLite database for backup purposes and also sends the data to a server that can perform technique classification. In the future, the LIFT automated weight lifting coach system could leverage many users using different sensors to contribute exercise data to help improve LIFT's classification algorithms.

\subsection{Data collection}

The YEI IMU is capable of collecting many different combinations of normalized, corrected, and filtered orientation and movement data. For v1 (version 1) of LIFT, the YEI IMU was configured to collect Euler angle and linear acceleration data processed by an on-chip Kalman filter at $20 \mathrm{~Hz}$. This combination of sensor data yielded what was thought to be the most relevant motion data for this application as explained in Chapter 3 [93]. In v2 of LIFT, the YEI IMU was configured to collect quaternion orientation, linear acceleration, corrected ac- 


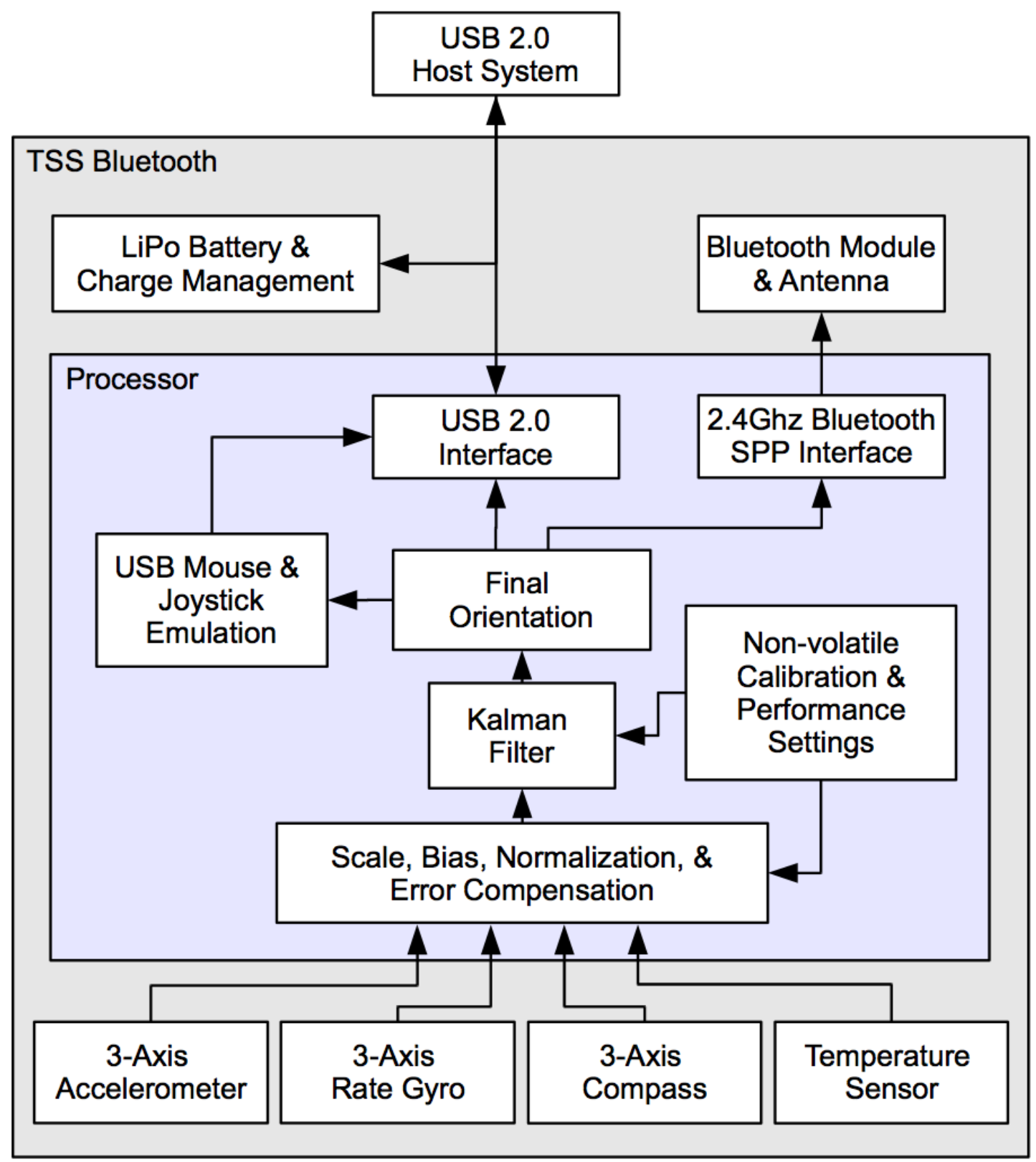

Figure 6.1: A system diagram of the YEI IMU [21]

celeration, corrected gyroscope, corrected magnetometer, raw acceleration, raw gyroscope, and raw magnetometer at roughly $70 \mathrm{~Hz}$. Many things were found to be broken after closely examining v1 of the LIFT automated weight lifting coach system. 
The YEI IMU records data in order to classify an athlete's technique for individual repetitions as seen in Figure 6.2. The individual repetitions are counted manually with the help of the data collection application. The manual accounting for each repetition ensures that data values recorded only from that particular repetition are used to derive the feature sets that the LIFT analysis system uses to classify techniques.
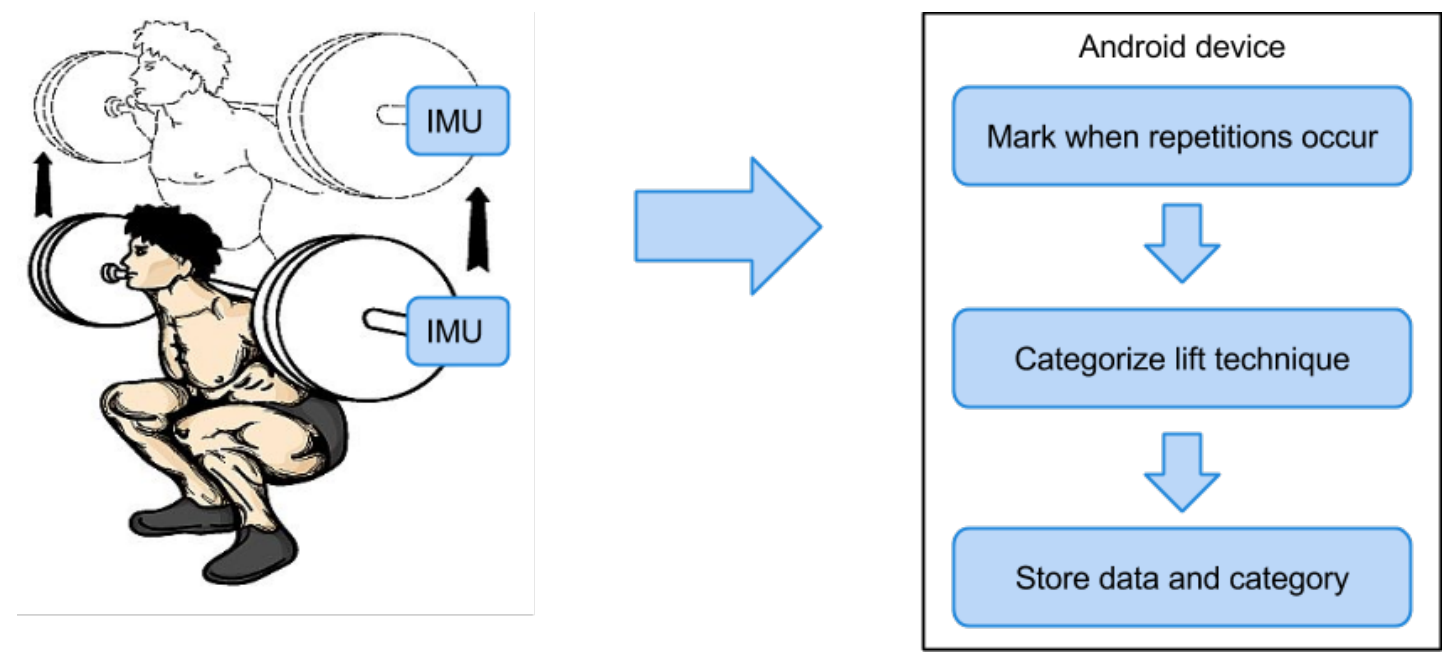

Figure 6.2: A system diagram of the data collection phase of LIFT

This is unlike Velloso et al.'s approach to classifying exercise techniques, where they used a sliding window approach in order to attempt to determine features of a particular repetition [110]. Since the Euler angle and linear acceleration data is known for a particular repetition, LIFT does not need to use a sliding window approach when calculating features of a repetition.

In the future, LIFT can incorporate more advanced repetition detection techniques to become a truly fully-automated weight lifting technique feedback system, but such algorithms are outside of the scope of this technique training and feedback study [87]. 


\subsection{Technique classification}

The LIFT analysis system uses Scikit-learn [86] classification algorithms to identify good and bad bar movement patterns, which are indicative of technique in the context of a particular exercise.

Each repetition was categorized in v1 by the quality of the bar's orientation by the recorded Euler angles and as well as the bar's path indirectly by linear acceleration. The quality of the bar's orientation is determined by the bar's roll, pitch, and yaw during a lift. There should not be any change in orientation of the bar in the lifts that LIFT is focusing on for this study: back squat, bench press, and overhead press. Therefore, any noticeable change in the bar orientation will be classified as a technique deficiency. The quality of the bar path is determined by linear acceleration felt by the sensor in the X-, Y-, and Z- axes as seen in Figure 6.3. The barbell should be moving in the X-Z plane in a controlled fashion for the back squat and bench press for particular reasons associated with each lift as discussed in Section 2.4. If a particular acceleration pattern is not seen, then the repetition should be classified as a technique deficiency. Whereas, for the overhead press, any significant acceleration in that plane should be classified as a technique deficiency.

After the repetitions were recorded, they were ran through the LIFT classification system, as seen in Figure 6.4. The system calculates features that describe what happens in each dimension in the orientation and linear acceleration vectors over the course of a repetition in v1, and additionally the corrected and raw accelerometer, gyroscope, and compass sensors in v2. The calculated features include: mean, variance, standard deviation, max, min, root mean square (rms), kurtosis, and skew. 


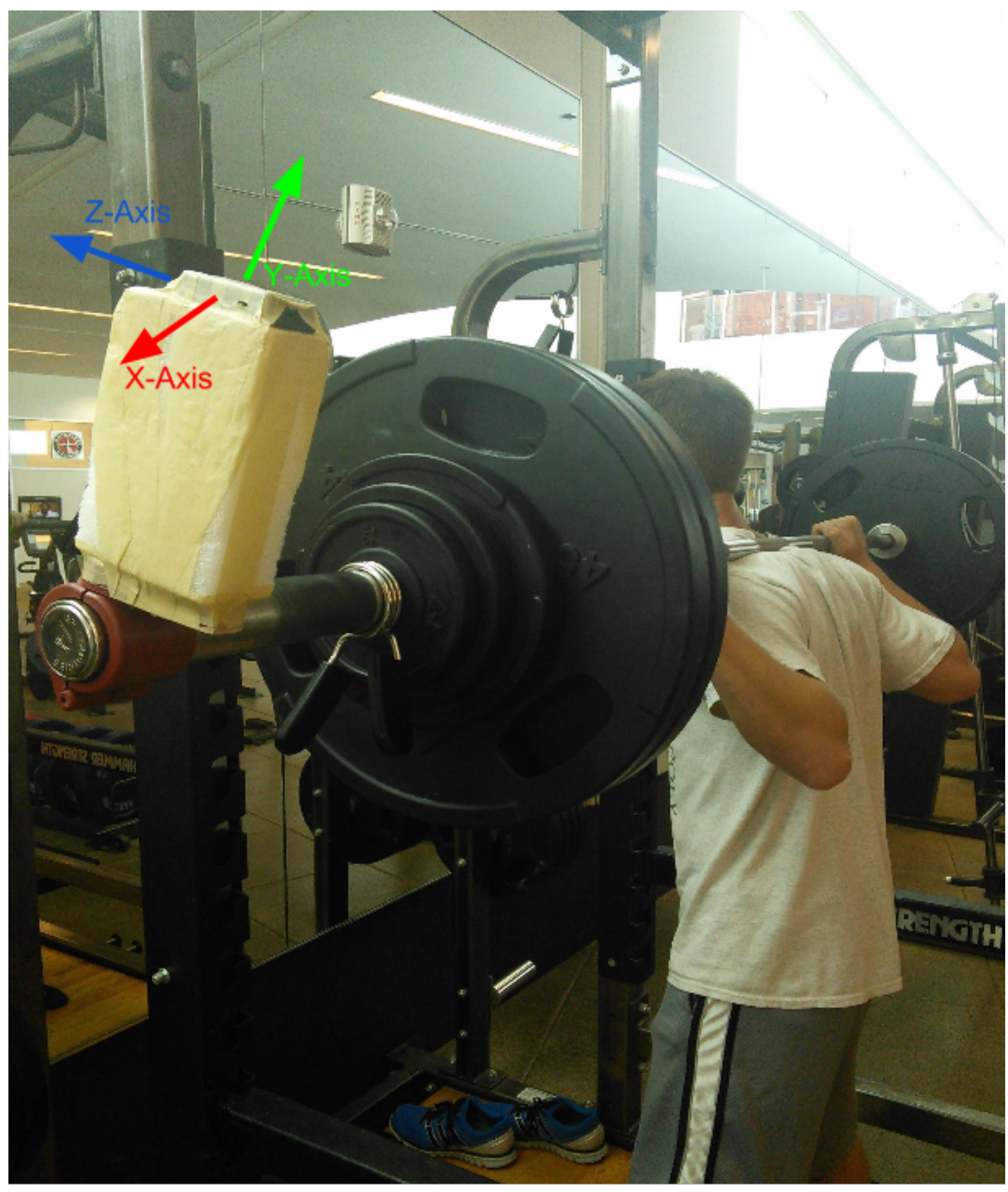

Figure 6.3: The axes of the YEI IMU when placed on a barbell

More advanced derived features are possible by obtaining velocity and position vectors. These are currently not in use due to the inherently noisy accelerometer signal. More complex filtering schemes are required to make use of these potential advanced features. Future devices, such as the Moov [11], seem to have built-in 

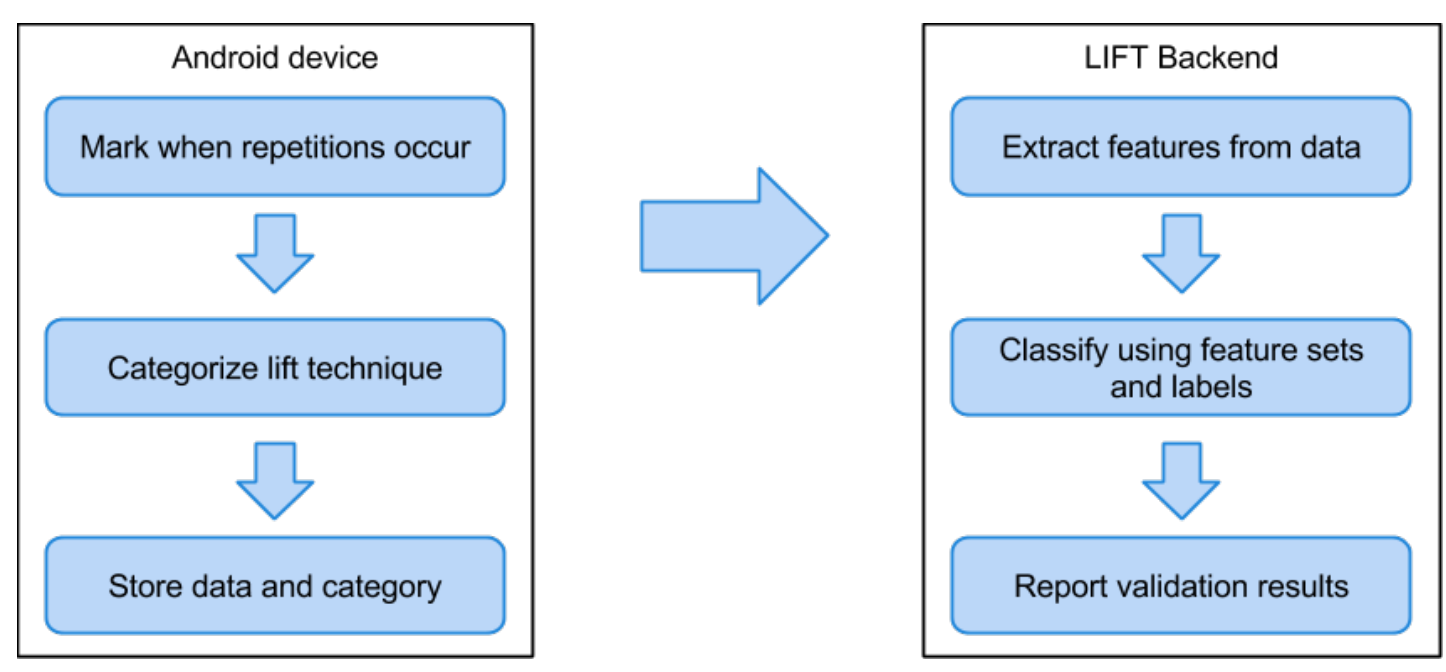

Figure 6.4: A system diagram of the technique learning phase of LIFT

position calculation, which could make these advanced derived features straight forward to calculate and train on.

For each repetition completed, there is a feature set that, in v1 of the design for the system, contains 48 derived values (6dimensions $\times 8$ features) with an associated label. In the second version, there are up to 200 derived values due to the extra corrected and raw data collected by the system (25dimensions $\times$ 8 features) The feature sets and associated labels were plugged into scikit-learn classification algorithms [86]. A battery of classifiers was used to initially see which would perform the best on the type of data LIFT was collecting. The types of classifiers used include: ensemble classification, support vector machines, as well as naive Bayes algorithms.

The ensemble algorithm, Extra Trees Classifier, performed the best with an 10 -fold cross validation accuracy of $83 \%$, with precision and recall around $81 \%$. The high cross validation and precision and recall numbers validated that the algorithm was able to determine the label for repetitions performed by athletes already in the data set. For athletes excluded from the data set, however, the 
algorithm only performed on average of $45 \%$ accuracy with leave-one-subject out validation for v1 of LIFT, but this was possibly due to a number of inherent faults in the data collection step. The leave-one-subject-out validation performed with an average of $41 \%$ accuracy with v2 of LIFT. This lower overall accuracy is possibly due to not enough trained athlete participants in the second study. These accuracies are further analyzed in Section 8

The Extra Trees classifier performed the best because it was able to form randomized decision trees that were not biased towards any one data label. As discussed in Section 3.3.3, the Extra Trees algorithm is able to build in more randomness in the decision tree formation process on top of the Random Forest approach. It not only randomizes the splitting mechanism, but it draws features from the entire feature set, unlike the Random Forest algorithm, which only draws a uniformly random features from the data set. This trade off decreases the variability of the resultant Extra Trees algorithm, but can increase the bias [47].

\subsection{Automated weight lifting coach}

The architecture of the LIFT coach can be seen in Figure 6.5. This automated method of providing feedback based on an existing data set of correct and incorrect lifts would be invaluable to helping a trainee perform proper technique on each repetition of their workout and promote long-term health by reducing the risk of injury during training. This architecture will be elaborated on further in Section 11 

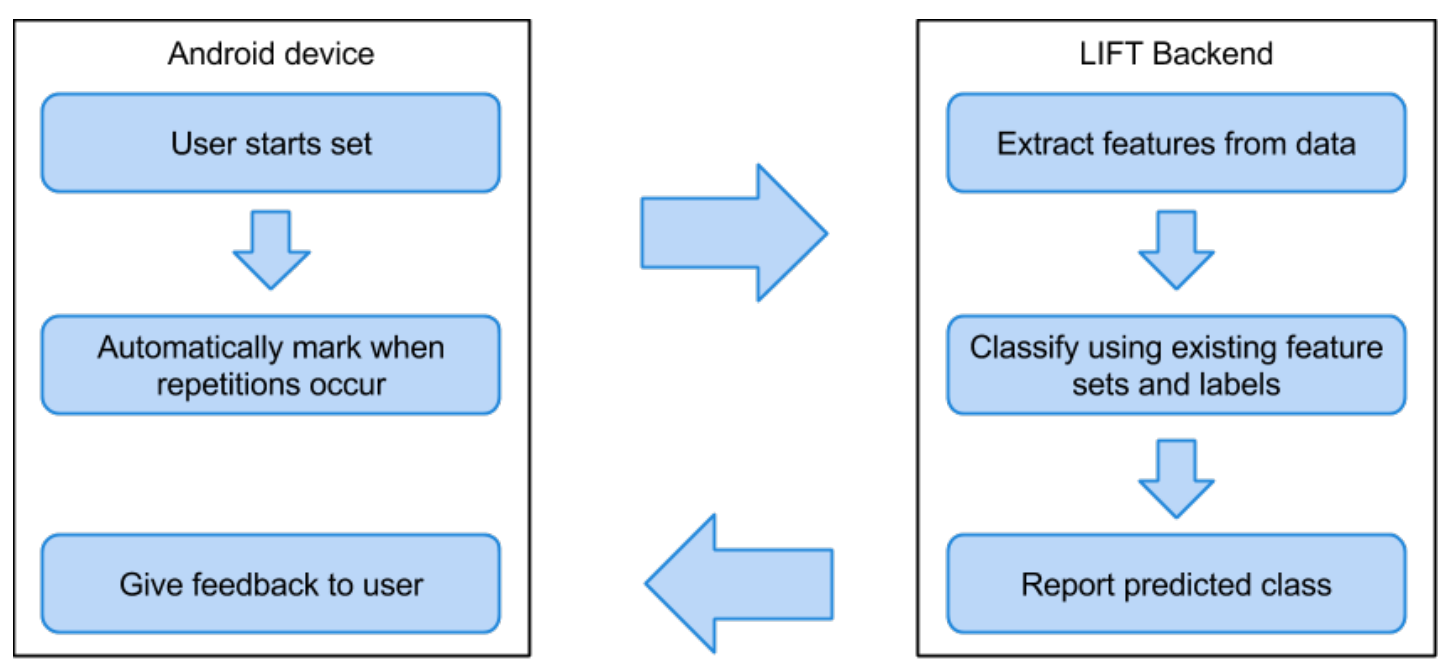

Figure 6.5: A system diagram of LIFT's future automated technique feedback loop 


\section{CHAPTER 7}

\section{Experimental Framework}

LIFT's Android mobile app was tested in Cal Poly San Luis Obispo's Athletic Weight Room and Recreation Center facilities. Cameron Van Wye, an athletic weight room strength and conditioning coach, supervised the athletes' technique as they performed strict overhead presses, back squat, and bench press with the Bluetooth-enabled IMU sensor attached to the left-side weight collar from the researcher's perspective as seen in Figure 6.3.

\subsection{Research protocol}

70 Cal Poly students participated in the study with LIFT v1, and 50 Cal Poly students participated in the study with LIFT v2. The athletes were tasked to perform 4 sets of 5 repetitions, working up to $75 \%$ of their 5 repetition maximum. This workload fatigued the athletes adequately enough to commit technique deficiencies, but not overly fatigue them. Van Wye discussed with the athletes what weight they felt comfortable with for 4 sets of 5 repetitions and chose the appropriate weight for them based on their comfort and strength levels.

Correct technique and technique deficiencies were observed by Van Wye while Lady operated the LIFT data collection app. LIFT's data collection functionality allowed Lady to record the start and end times of repetitions and label the repetitions as observed by Van Wye. 
Individual repetitions were counted by the researchers at the bottom (after the eccentric) portion of the overhead press, and at the top (after the concentric) portion of the squat and bench press. This definition of a repetition enabled the researchers to clearly communicate when technique deficiencies started to occur and record a consistent window of data values (orientation and linear acceleration vectors). The LIFT analysis system then derives aggregate features from these data values.

\subsection{Validation}

Leave-one-subject-out validation was used as the primary means for determining LIFT's performance. Leave-one-subject-out emulates the performance of the LIFT system in a live technique feedback situation for each recorded athlete's completed repetitions.

This form of validation goes through each athlete contained in the data set and excludes or "holds out" their exercise repetition data from being used to train the technique classifier. Once the technique classifier has been trained, the excluded or "held out" data is then run on the technique classifier. The performance of the classifier is based on how many repetitions it is able to label correctly out of the total amount of repetitions for the athlete. The performance of the system as a whole is judged by aggregating all of the results for each athlete excluded from the data set.

This type of validation ensures that the information gained by the classification algorithm is applicable and valid for classifying an athlete's weight lifting technique who is not in the current data set. A $70-80 \%$ leave-one-subject-out accuracy would be comparable to Velloso's leave-one-subject-out accuracy [110]. 


\section{CHAPTER 8}

\section{Results}

Overall, the LIFT coach was not able to accurately distinguish individual technique deficiencies at a commercial production-quality level where a trainee could rely on LIFT to recognize and help correct potentially dangerous technique deficiencies. In v1, a number of researcher errors were the main cause behind the low leave-one-subject-out accuracies. These adjustments between v1 and v2 are discussed in Analysis in Section 9. Low leave-one-subject-out scores are still prevalent in v2 and seem to primarily be caused by a lack of a "balance" of data, where the training data set would contain a roughly equal distribution of highly trained athletes and novice weight lifters. "Simple" confusion matrices have been calculated to show that in the simple case, LIFT could indicate to the trainee that something is likely wrong with their technique and can walk the trainee through a standard procedure to remind the user of the correct technique. Although this "simple" methodology was not in the intended goal, this is a good first step towards validating that this type of technique evaluation system is feasible and possibly useful.

In an attempt to gain a better general understanding of how v2 has improved over v1, "simple" confusion matrices have also been included in the tabular and graphical results. These simple matrices condense all of the technique deficiency labels of a given exercise to just "Incorrect" to be compared to when the technique is "Correct". 
The following results of the LIFT coach are normalized with respect to the "truth label" rows, so they are able to be easily compared to Velloso et al.'s results [110]. Confusion matrix magnitude data is displayed in non-zero cells to compare the number of times a given label was recorded to other labels. "Golden" repetitions were very close to ideal demonstrations of weight lifting technique. They would be suitable to use as a base for a "golden standard" method of technique deficiency detection, as discussed in Section 11.6, but is not relevant for classification purposes, so its "deficiency priority" weight is low. The higher the priority label, the more central to the movement it is. For example "Lower back round" has a very high priority for squatting because it is a technique deficiency that can have a high risk of injury at heavy loads and should be corrected above all other deficiencies in the squat.

One of the issues that has affected this study is the researchers not collecting data evenly across all labels so they can be compared fairly against each other for classification. A label with more examples than other labels is more likely to bias a classifier and cause the classifier to "overfit" its decision-making structure for that overrepresented label.

\subsection{Squat results}

The squat is a fundamental weight lifting movement that is straight forward to learn, but difficult to master. Due to the nature of the lift, there are many possible technique deficiencies that a trainee can commit. Many individual technique deficiency repetitions were recorded during v1 and v2 testing, but only a few different types of technique deficiencies were recorded a significant number of times to be recognized adequately by the classifier. This fact can be seen in 
Figure 8.1 where there are only two labels along the diagonal that have an above $50 \%$ recognition rate, in this case "Correct" and "Did not reach parallel". It is interesting to note on this figure as well that "Upper back round", "Knees not spread out", "Wrists rolled back", "Chin not tucked", "Heels coming up off of ground", "Lower back round", and "Hips roll under spine" get confused with "Correct" technique very often in this data set. This may be due to the combination that these technique deficiencies do not cause out of the ordinary bar path features, and that they had much fewer recorded repetitions than the "Correct" label. This trend translates into the v1 simple confusion matrix in Figure 8.2, where the "Correct" label dominates and causes "Incorrect" feature sets to be classified as "Correct".

The second iteration of LIFT was able to capture a wider variety of technique labels due to primarily testing LIFT in the Cal Poly Recreation center, but at the cost of not capturing as many "Correct" labels. This is opposed to the first iteration where testing took place primarily in the Cal Poly Athletic weight room and because the student-athletes are much more well-trained, they committed fewer technique deficiencies, resulting in more "Correct" labels.

In v2 squat testing, as seen in Figure 8.3, "Did not reach parallel", and "Correct" were the most successful predicted labels for squatting. "Did not reach parallel" often got confused as "Correct" and vice versa, which is not unexpected because a trainee could have leg and torso lengths than another trainee. These different limb proportions will define different distances the that the bar must travel to reach the parallel mark. LIFT does not use estimated displacement as a feature, but the researchers hypothesize that coming up out of a half squat will look different than coming up out of a full squat with respect to Linear Acceleration on the Y-axis, which is possibly why there was some moderate success in 
distinguishing between the two labels.

Another notable mislabeling in the v2 squat testing was "Lower back round" being mistaken for "Did not reach parallel". These labels were the most common type of technique deficiencies seen in the Cal Poly Recreation Center, and are generally committed in a mutually-exclusive fashion. "Lower back round" typically takes place when the trainee is trying to achieve a full, below-parallel, "ass-to-grass" squat, but they do not have the hamstring flexibility, ankle mobility, or the awareness to keep a neutral spine throughout the lift, etc. When the trainee is about to reach parallel or lower, their pelvis starts to tilt in a posterior fashion in order to allow the body to continue the motion downwards. When this motion is unloaded, it is harmless. But when the trainee is squatting with their working set weight, the load can cause the lower back vertebrae to experience a large amount of shear stress and "pinch" together, resulting in injury. What makes this misclassification interesting is that when the trainee does not go to parallel, and therefore are nowhere near rounding their lower back. This misclassification shows that inferring body positioning that isn't directly in contact with the bar based on bar movement is a hard problem to solve.

In the simple squat confusion matrix for v2, Figure 8.4 LIFT is able to distinguish incorrect technique from correct technique with some success, but not quite enough for production-level "live" coaching for specific technique deficiencies. As stated before, these levels If the researchers were able to collect more correct technique data so the data set was not as skewed towards incorrect technique, it would be interesting to see if there would still be a modest

Overall, v2 improved upon v1's results for the simplified confusion matrices 8.1. v2 had a decline in performance from v1 in the full confusion matrix because there were a wider variety of recorded labels to classify, so the comparison is not 
entirely fair. The researchers expect that if $\mathrm{v} 1$ were exposed to as many different technique deficiencies as v2 that its performance would be the same as v2's or worse due to factors that will be covered in Section 9 .

$\begin{array}{llll} & \text { Accuracy (\%) } & \text { Precision (\%) } & \text { Recall (\%) } \\ \text { Squat v1 full } & 54.9 & 28.8 & 17.9 \\ \text { Squat v1 simple } & 58.5 & 58.4 & 56.7 \\ \text { Squat v2 full } & 35.7 & 36.7 & 25.6 \\ \text { Squat v2 simple } & 70.2 & 63.9 & 68.1\end{array}$

Table 8.1: Comparison of squat measures between v1 and v2

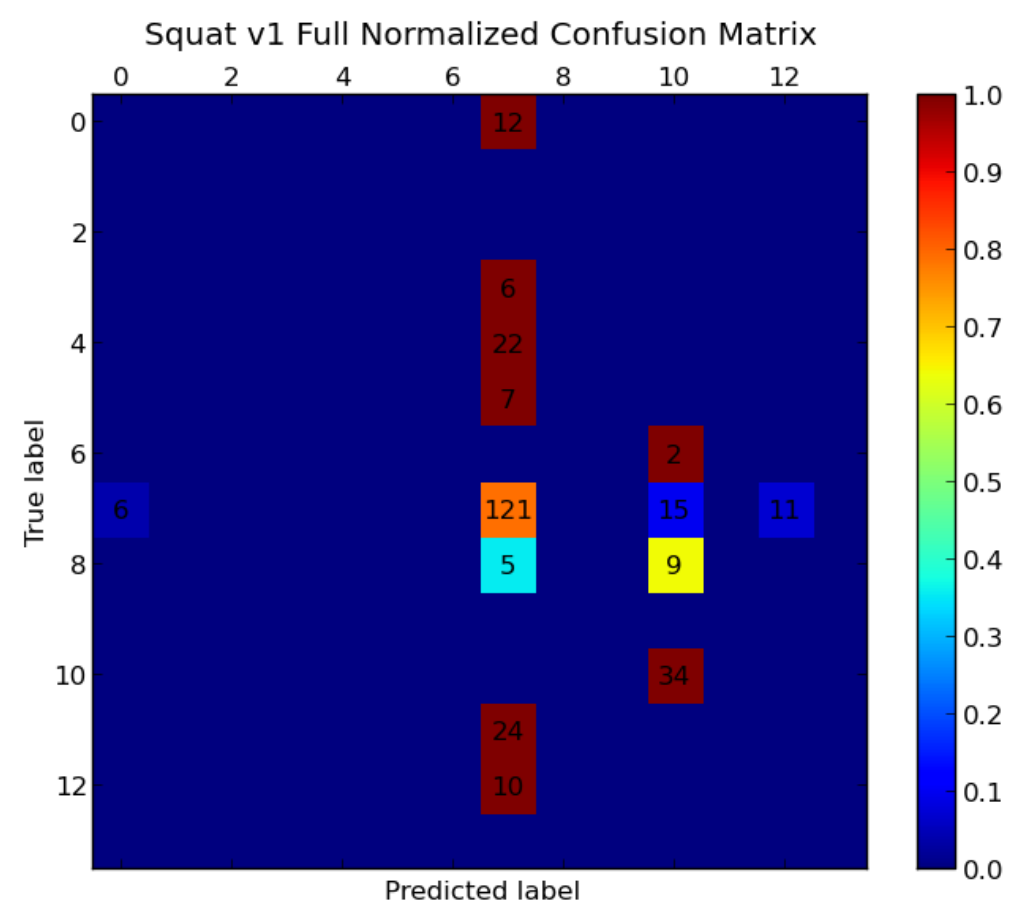

Figure 8.1: Full normalized confusion matrix for v1 squat 


$\begin{array}{lll}\text { Label number } & \text { Squat Labels v1 } & \text { Squat Labels v2 } \\ 0 & \text { Upper back round } & \text { Did not stand all the way up } \\ 1 & \text { Over extension / too vertical } & \text { Upper back round } \\ 2 & \text { Jerky rep } & \text { Over extension / too vertical } \\ 3 & \text { Knees not spread out } & \text { Hips out / chasing with back } \\ 4 & \text { Wrists rolled back } & \text { Knees not spread out } \\ 5 & \text { Chin not tucked } & \text { Wrists rolled back } \\ 6 & \text { Chasing with back } & \text { Chin not tucked } \\ 7 & \text { Correct } & \text { Jerky rep } \\ 8 & \text { Heels coming up off of ground } & \text { Correct } \\ 9 & \text { Golden } & \text { Heels coming up off of ground } \\ 10 & \text { Did not reach parallel } & \text { Golden } \\ 11 & \text { Lower back round } & \text { Did not reach parallel } \\ 12 & \text { Hips roll under spine } & \text { Lower back round } \\ 13 & \text { Did not complete repetition } & \text { Hips roll under spine } \\ 14 & \text { N/A } & \text { Did not complete repetition }\end{array}$

Table 8.2: Squat label number table

\subsection{Bench press results}

The bench press is by and large the most recognizable and popular barbell exercise. Because of its widespread popularity, lots of trainees can be under-coached about how to properly perform the bench press. The first version of LIFT predicted the labels "Upper back not tight / elbows out" and "Correct" with some success. "Upper back not tight / elbows out" was often misclassified as "Correct" or "Wrists rolled back" as well. The "Upper back not tight / elbows out" label is used when a trainee flares their elbows out towards 90 degrees, almost perpen- 


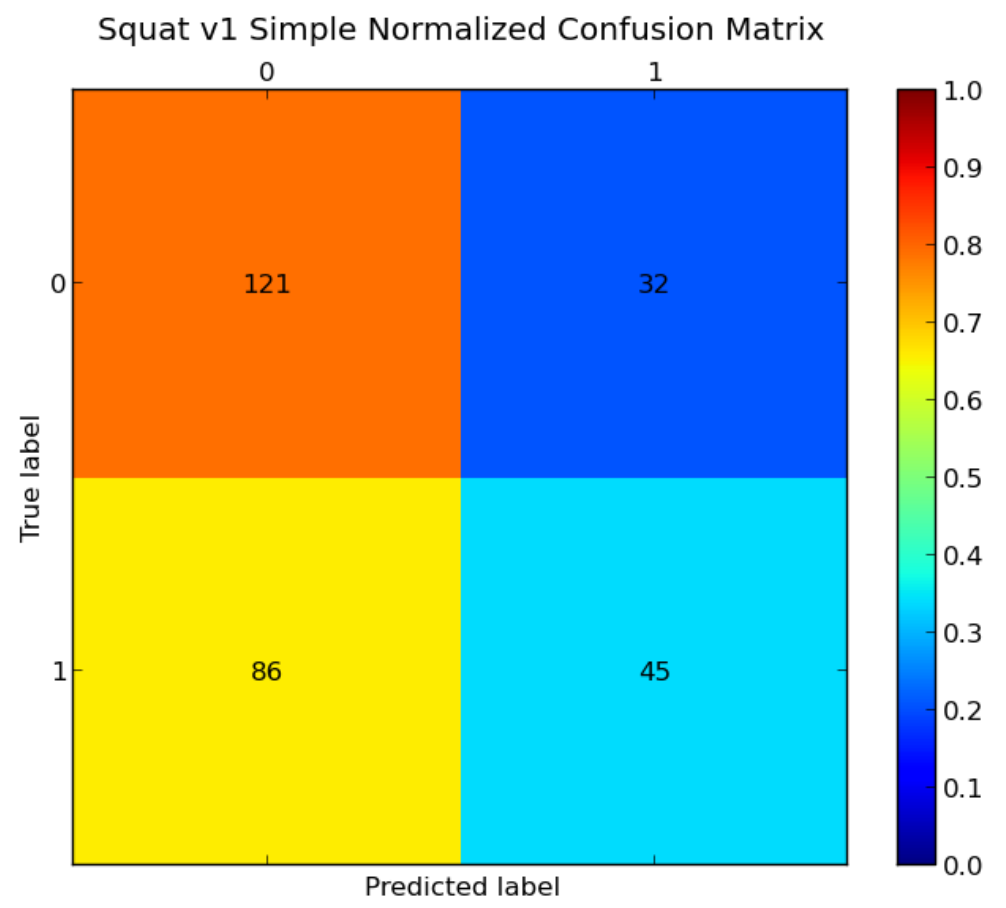

Figure 8.2: Simple normalized confusion matrix for v1 squat

dicular with their torso. While this may create a more efficient bar path, it can put the shoulder joint at a higher risk for impingement, as seen in Figure 2.7. Elbows tucked in closer, at around 45 degrees from the trainee's torso is an acceptable compromise between risk of injuring the shoulder and efficient bar path. The "Upper back not tight" portion of the label is the method that the trainee will typically use to keep their elbows from flaring out. The trainee can contract their lats and create tension that helps stabilize their back on the bench and keeps their elbows pulled in. Given that background, it is somewhat encouraging to see that the "Upper back not tight / elbows out" label can be differentiated from "Correct" technique more than half of the time, but "Correct" technique is more often than not mistaken for "Upper back not tight / elbows out". This could be an acceptable misclassification if the trainee is a novice and needs to 


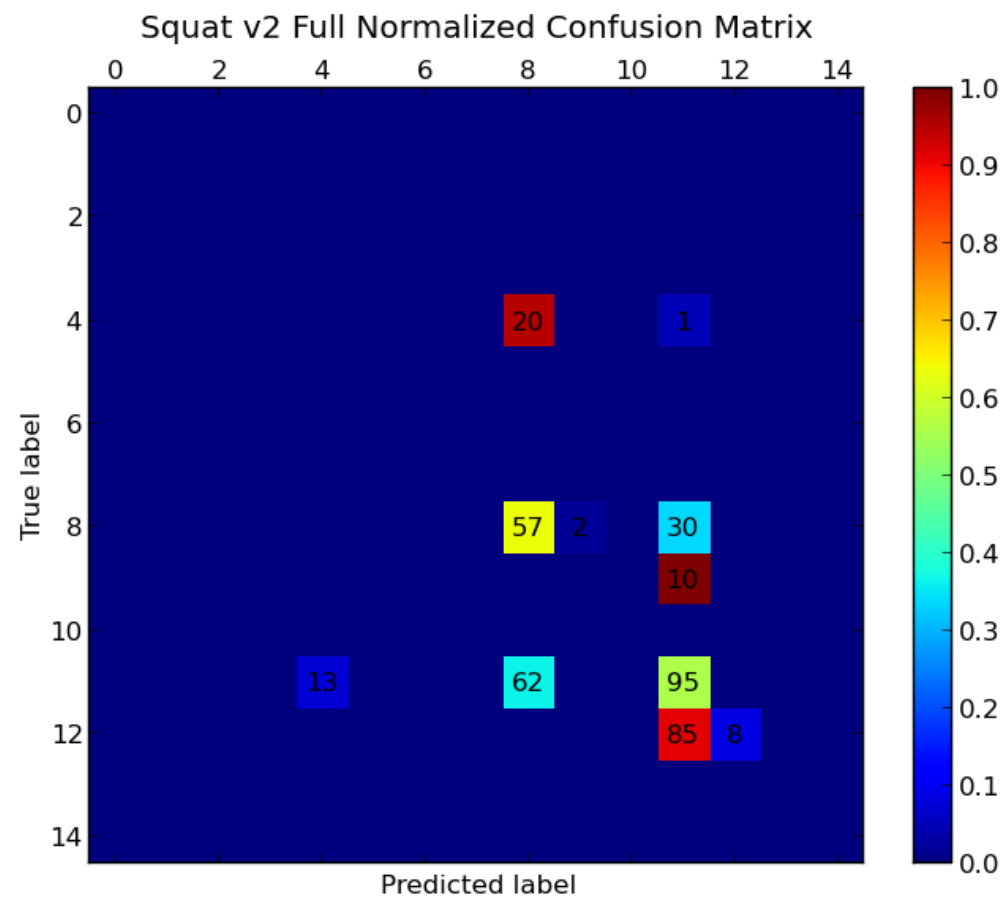

Figure 8.3: Full normalized confusion matrix for v2 squat

consistently be reminded to pull their elbows in, regardless if their elbows were flaring out or not. This type of feedback to an experienced lifter who has their technique completely drilled into their thought process, may be extraneous and annoying because they would notice their own elbows flaring out on a lift more accurately than LIFT could predict.

LIFT did a fairly poor job of detecting the "Wrists rolled back" label. Although the Euler angle orientation measurements that were recorded could have accurately captured this label, it was more of a transient technique deficiency and did not have many total repetitions, so there weren't many good teaching opportunities for LIFT to learn from the feature sets associated with that label. The "Glutes not engaged" label has a similar explanation as Section 8.1's "Lower back round" and "Did not reach parallel" labels: the glutes are only indirectly in- 


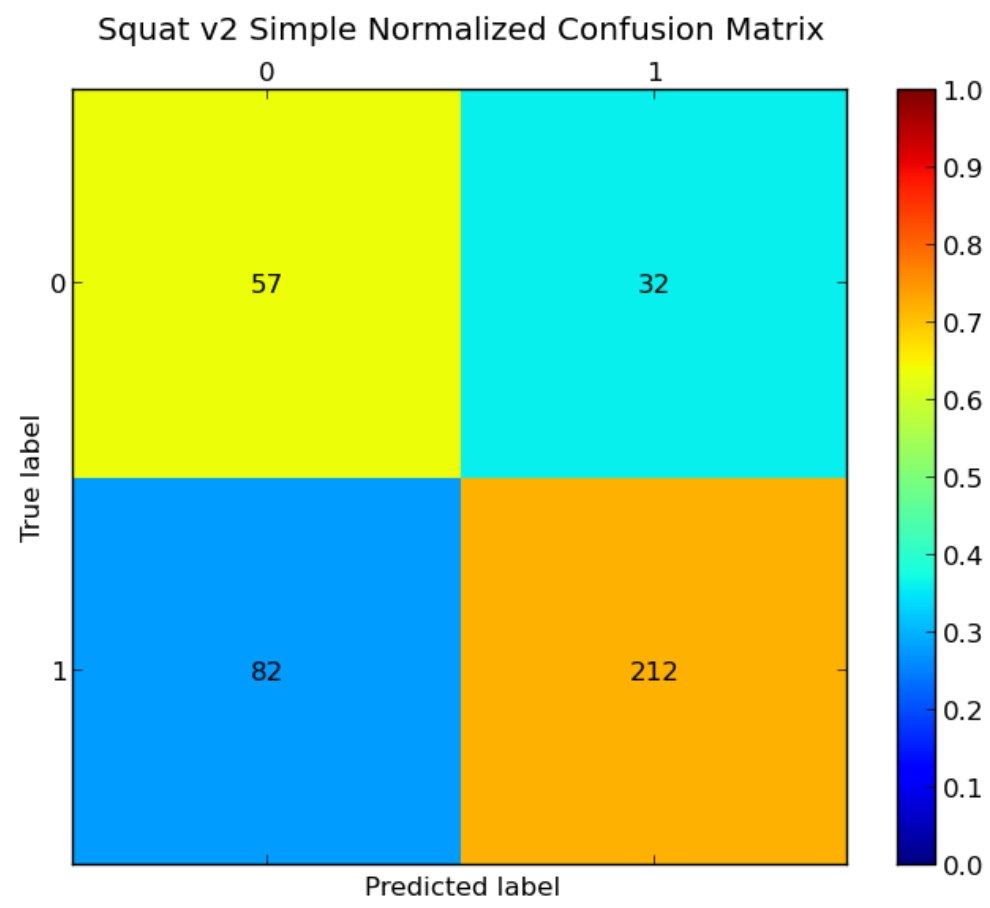

Figure 8.4: Simple normalized confusion matrix for v2 squat

fluencing the bar path by supporting the torso by keeping it stable on the bench, unlike "Upper back not tight / elbows out" or "Wrists rolled back", which each would be detected by their direct effect on the bar path.

The simplified v1 confusion matrix for bench press as seen in Figure 8.6 would not inspire confidence in the novice trainee. LIFT would more often than not encourage the trainee to remember proper technique and have the trainee go through the steps to setting up a correct bench press, regardless if they had just correctly performed bench press. If the trainee wanted to get validation that their bench press technique was good, they would not find v1 of LIFT very helpful.

The second iteration of LIFT's bench press technique detection, as seen in Figure 8.7, is still moderately successful at detecting when a trainee's elbows are out while their bench pressing, but the overfit classifier mistakes large numbers 
of the labels "Did not lock out", "Bounce off of chest", "Correct", "Upper back not tight", "Excessive lower back arch", and "Did not touch chest" as "Elbows out". This is probably mostly due to the researchers collecting lots of data in Cal Poly's Recreation Center and not balancing the time between there and the Cal Poly Athletic weight room collecting data on athletes who are very proficient at performing correct bench press technique. This is the primary cause of why the full precision and recall for v2 as seen in Table 8.3 is very poor, worse off when compared with v1 of bench press data.

The great accuracy, precision, and recall numbers for LIFT's bench press technique detection on the v2 simple confusion matrix 8.6 are misleading, primarily because there are just so many incorrect labeled repetitions in comparison to correctly labeled repetitions. To be fair, the researchers were primarily interested in figuring out if LIFT could differentiate between specific technique deficiencies and give the trainee specific feedback about how to most accurately and effectively fix their barbell lifts. It was not until far into the research that it was suggested that LIFT could simply determine if a given repetition was simply correct or incorrect. If this information alone was very accurate, this would be fairly useful tool for novice trainees to have a reminder to concentrate on good technique above all else and would positively reinforce them to know what good technique feels like and also what it feels like when limbs are out of sync.

\subsection{Overhead press results}

The first version of LIFT's overhead press technique detection was able to correctly identify correctly labeled repetitions fairly successfully 8.9. This comes at a cost of biasing LIFT to misclassify techniques such as "Elbows out", "Jerky 


\section{Accuracy (\%) Precision (\%) Recall (\%)}

$\begin{array}{lccc}\text { Bench v1 full } & 39.4 & 20.9 & 14.7 \\ \text { Bench v1 simple } & 57.7 & 48.1 & 48.2 \\ \text { Bench v2 full } & 46.4 & 13.4 & 13.9 \\ \text { Bench v2 simple } & 78.8 & 63.9 & 47.9\end{array}$

Table 8.3: Comparison of bench press measures between v1 and v2

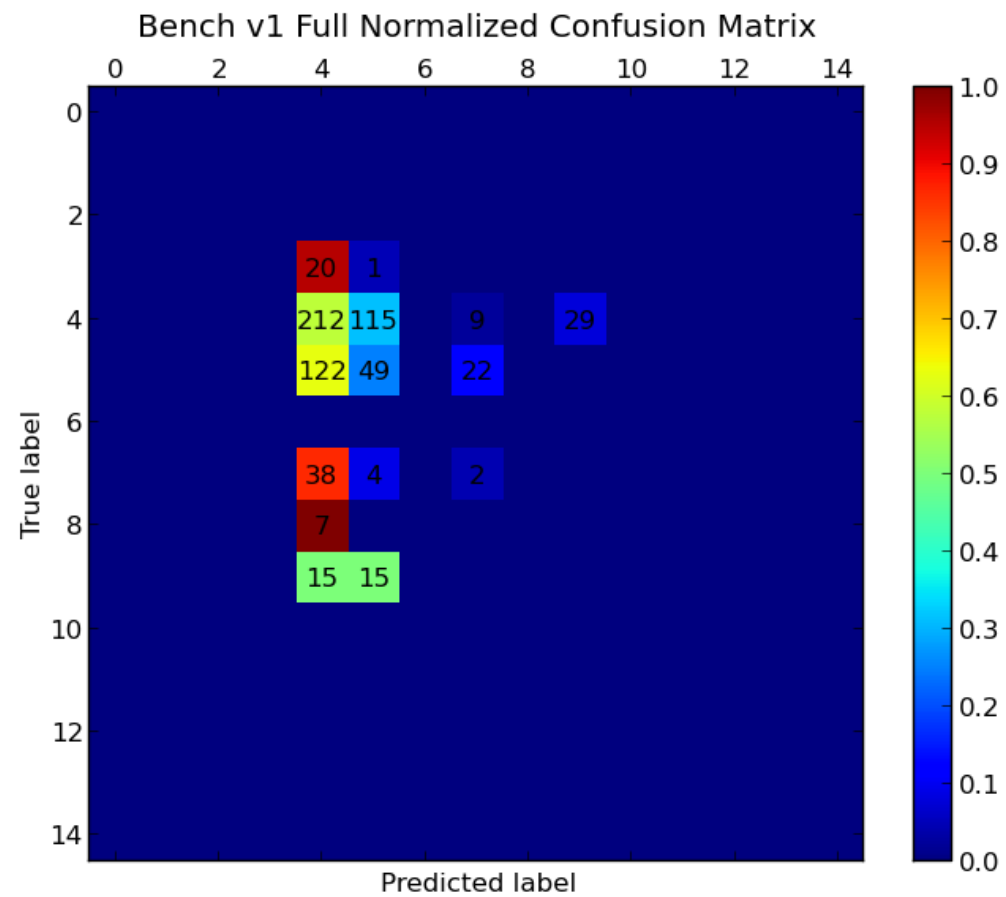

Figure 8.5: Full normalized confusion matrix for v1 bench press

repetition", "Didn't get under bar", "Wrists rolled back", "Too much layback", and "Didn't use hips" as "Correct". This is a result of, very much like squat and bench press, primarily testing on well-trained athletes for v1 and recording a large number of "Correct" labeled feature sets. "Elbows out", "Jerky repetition", "Wrists rolled back", and "Too much layback" labels were able to find some small success in being correctly classified, but their success was very limited due to the 


$\begin{array}{lll}\text { Label number } & \text { Bench Label v1 } & \text { Bench Label v2 } \\ 0 & \text { Jerky repetition } & \text { Elbows out } \\ 1 & \text { Did not complete repetition } & \text { Jerky repetition } \\ 2 & \text { Did not lock out } & \text { Did not complete rep } \\ 3 & \text { Bounce off of chest } & \text { Did not lock out } \\ 4 & \text { Upper back not tight / elbows out } & \text { Bounce off of chest } \\ 5 & \text { Correct } & \text { Correct } \\ 6 & \text { Golden } & \text { Golden } \\ 7 & \text { Glutes not engaged } & \text { Upper back not tight } \\ 8 & \text { Excessive lower back arch } & \text { Glutes not engaged } \\ 9 & \text { Wrists rolled back } & \text { Excessive lower back arch } \\ 10 & \text { Left side came up first } & \text { Wrists rolled back } \\ 11 & \text { Did not touch chest } & \text { Left side came up first } \\ 12 & \text { Right side came up first } & \text { Did not touch chest } \\ 13 & \text { N/A } & \text { Right side came up first }\end{array}$

Table 8.4: Bench press label numbers

overwhelming bias towards "Correct" technique labels.

The simplified v1 version of press technique detection for LIFT, as seen in Figure 8.10 is successful more than half of the time at differentiating "Correct" techniques from "Incorrect" techniques, and just about half of the time for the other way around. LIFT as an overhead press coach could help validate that a novice user was correctly performing the lift half of the time, but during the other half of the time they would get feedback to remind themselves about how to properly perform overhead press. This could be useful for novices who need to constantly be reinforced and encouraged that they are performing correct or 


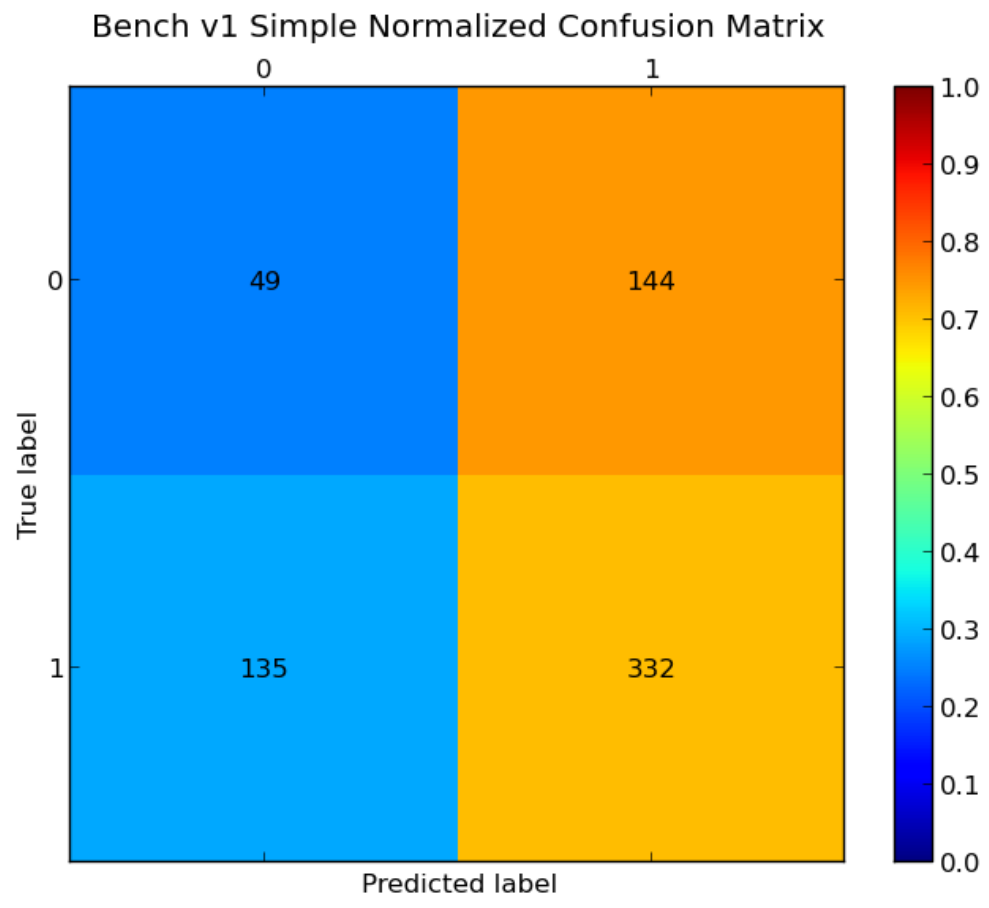

Figure 8.6: Simple normalized confusion matrix for v1 bench press

incorrect technique. LIFT can be a useful tool in this way until trainees develop a sense for knowing what is going on in their technique and when something is wrong.

The LIFT coach was not able to be trained for strict overhead press in the second iteration due to a shortage of trainees in the recreation center who performed overhead press during the data collection periods. 


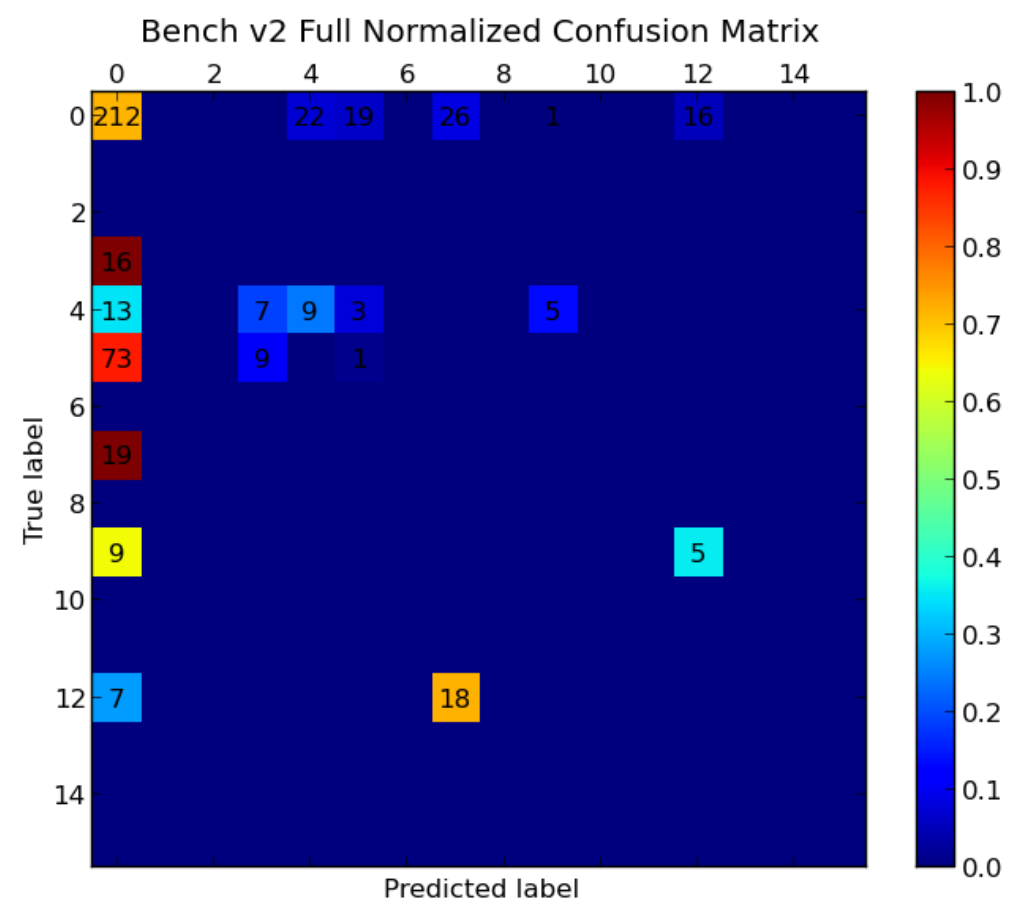

Figure 8.7: Full normalized confusion matrix for v2 bench press

$\begin{array}{llll} & \text { Accuracy (\%) } & \text { Precision (\%) } & \text { Recall (\%) } \\ \text { Press v1 full } & 44.8 & 21.2 & 20.3 \\ \text { Press v1 simple } & 56.4 & 48.1 & 56.1 \\ \text { Press v2 full } & \text { N/A } & \text { N/A } & \text { N/A } \\ \text { Press v2 simple } & \text { N/A } & \text { N/A } & \text { N/A }\end{array}$

Table 8.5: Comparison of overhead press measures between v1 and v2 
Bench v2 Simple Normalized Confusion Matrix

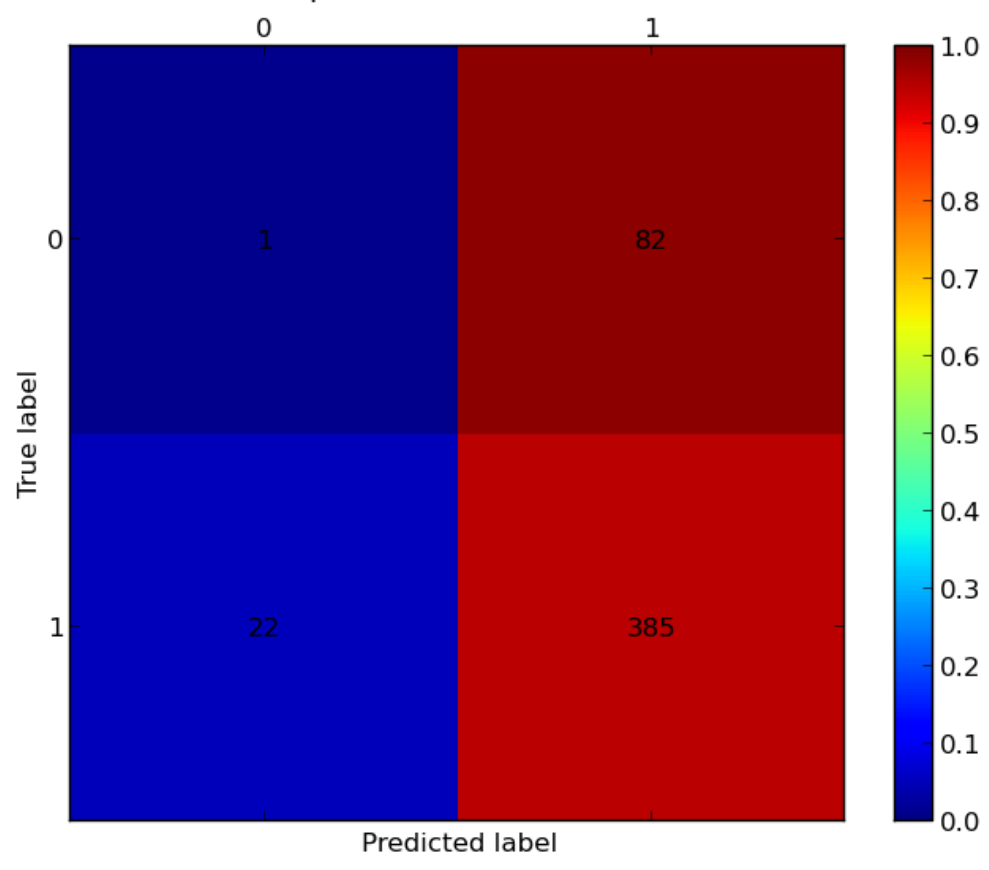

Figure 8.8: Simple normalized confusion matrix for v2 bench press 


$\begin{array}{ll}\text { Label number } & \text { Press Label v1 } \\ 0 & \text { Elbows out } \\ 1 & \text { Pushed bar away / started too far out } \\ 2 & \text { Chest/Shoulders/Elbows down } \\ 3 & \text { Did not complete rep } \\ 4 & \text { Jerky repetition } \\ 5 & \text { Right side came up first } \\ 6 & \text { Didn't get under bar } \\ 7 & \text { Correct } \\ 8 & \text { Golden } \\ 9 & \text { Wrists rolled back } \\ 10 & \text { Too much layback } \\ 11 & \text { Head down } \\ 12 & \text { Left side came up first } \\ 13 & \text { Didn't use hips }\end{array}$

Table 8.6: Overhead press v1 label numbers 


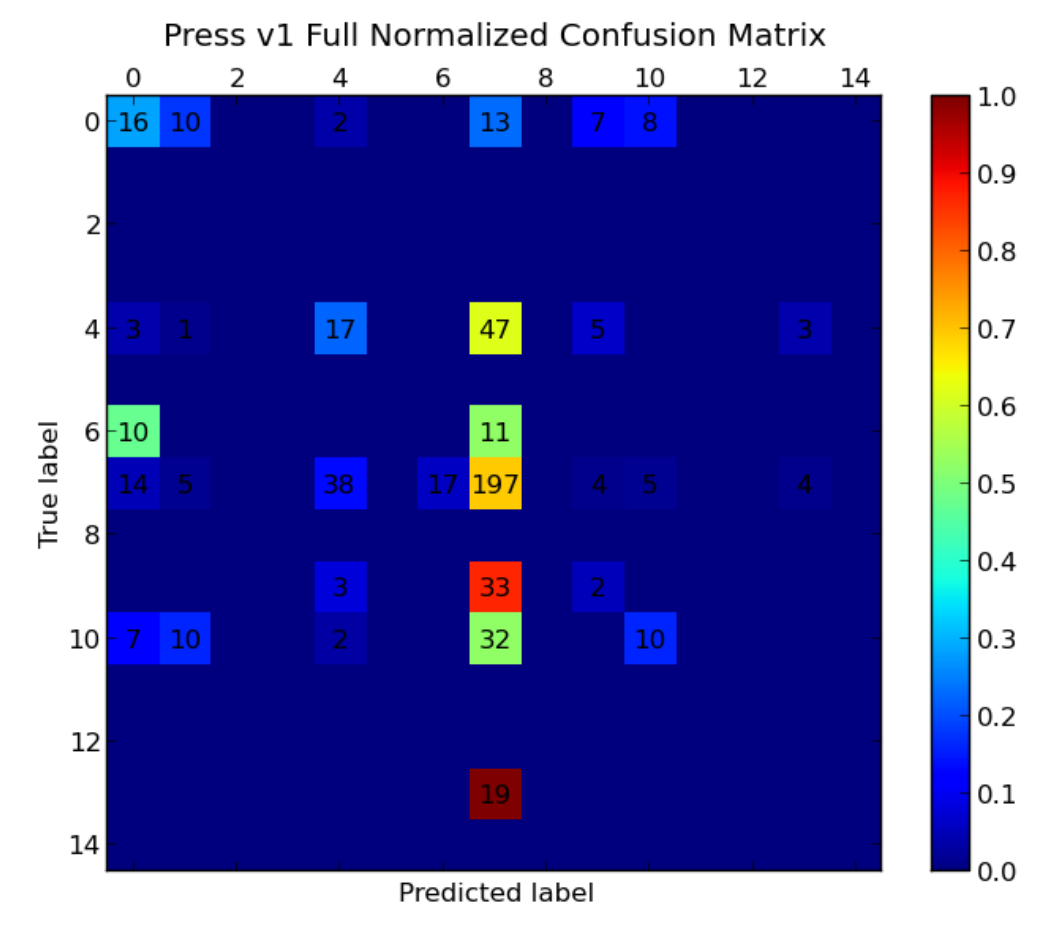

Figure 8.9: Full normalized confusion matrix for v1 overhead press 


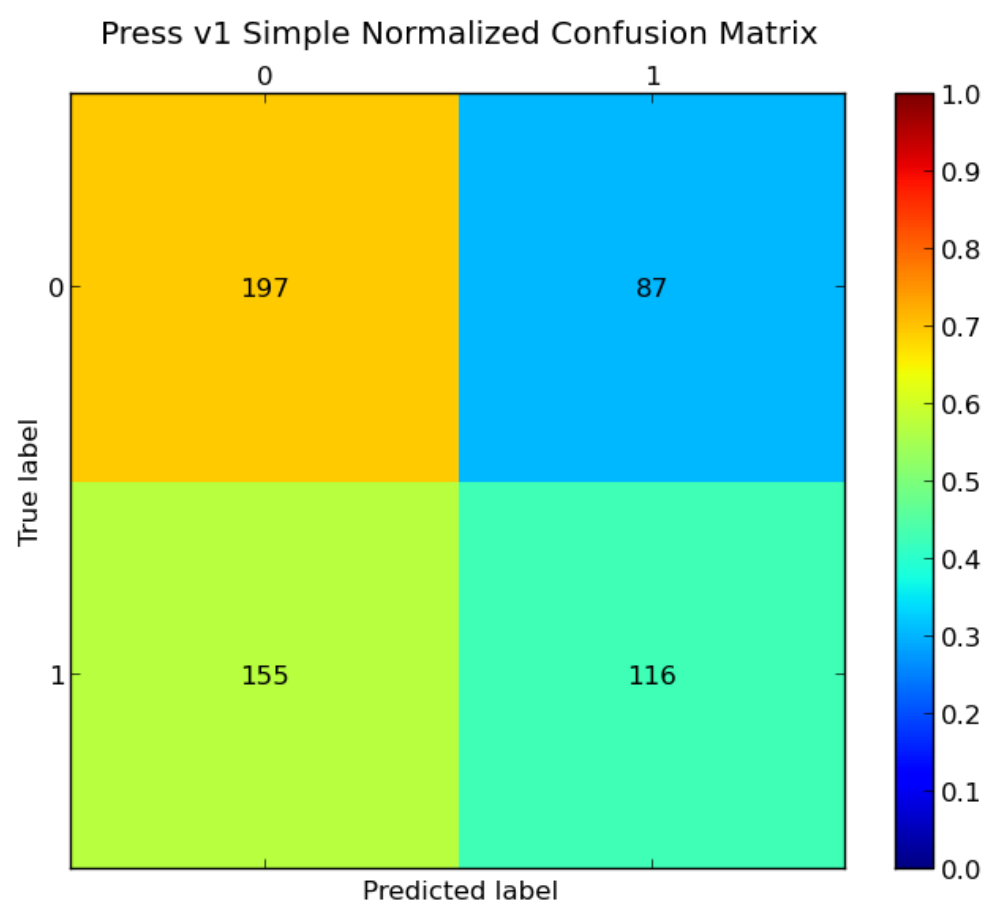

Figure 8.10: Simple normalized confusion matrix for v1 overhead press 


\title{
CHAPTER 9
}

\begin{abstract}
Analysis
Deriving results from the LIFT coach was an iterative process. Due to the nature of running a human subject experiment and capturing "live" data, testing revealed that the data collection system, study methodology, and technical design error all played a part in obtaining the first, fairly erroneous results from LIFT.
\end{abstract}

\subsection{Research study bias and error}

As with any data collection experiment, unexpected things happen that help correct and steer the direction of the experiment towards better definitions, measurements, and representative participant demographics.

\subsection{Athlete bias}

The athletes in the weight room are well-trained, so they did not commit many technique deficiencies, so the number of correct repetitions in the training set are disproportionately higher than the rest of the repetition labels. This also affected the leave-one-subject-out validation as well. If only one subject committed a technique deficiency or a limited number of repetitions across multiple subjects were labeled as a particular deficiency, then they were very likely to be misclassified as another technique deficiency or as a correct repetition. 


\subsection{Technique deficiency challenges}

At various points during the study, subjects performed weight lifting exercises in such a way that did not fall under pre-existing labels for technique deficiencies, so a new labels had to be created and accounted for. On the other end of the technique deficiency labeling spectrum: subjects either had a very different understanding of what an exercise entailed, or were very undertrained, but had a correct understanding of what they should be attempting to accomplish for a given exercise. Both of these personas introduced the notion of labeling a repetition with multiple technique deficiencies. Recording multiple labels for a specific repetition makes algorithmic classification a very hard problem.

\subsection{Researcher error}

The researchers were not perfect in identifying exactly when the repetitions started and ended or what its particular label was with LIFT's data collection app, occasionally misclicking and missing a repetition, cutting off a repetition too quickly, or not remembering when exactly a subject's technique started to break down. These error sets were later added to a blacklist to be ignored when analyzing the data in LIFT, but not all of the error sets were blacklisted. This introduces a small amount of error, limited to 1 - 5 repetitions per testing day,

which is significantly less than 2833 which is the amount of repetitions recorded in total across both versions of the study. The mislabeling types of errors occurred when subjects performed repetitions greater than or equal to the average size of the researcher's short-term memory (5 - 10 repetitions) [75]. 


\subsection{Overcoming research study bias and error}

To combat these research study-specific sources of bias and error for athlete bias, challenges with specific weight lifting techniques, and researcher recording error, respectively, the researchers done in turn:

1. Received approval from the Human Subjects committee to conduct their study in the Cal Poly Recreation Center, where less-well trained athletes are more likely to commit technique deficiencies on their own.

2. Amended their testing protocol to explain and demonstrate what they mean when they instruct the subject to perform a specific exercise.

3. Limited the number of repetitions athletes perform to 5 , because it is easier to remember and break down exactly when an exercise started to be performed poorly.

These study improvements made the data collection process run much smoother and enabled the researchers to collect data more accurately.

\subsection{Technical error considerations}

The first iteration of trainee testing with LIFT was unfortunately inherently flawed in multiple facets, starting at the sensor level.

\subsubsection{Orientation taring}

There was an error in the data collecting app where the orientation was automatically tared at the start of every set, regardless of the sensor's actual orientation. This was not a correct taring method because the YEI documentation states that the sensor considers "the zero orientation to be the orientation in which the plug 
is facing towards you and top(the side with buttons on it) facing up" [22]. The reasoning behind this specific zero orientation is that the "sensor must be given a reference orientation that represents the orientation of the sensor when it is in the position in which you consider the plug to be towards you and the buttons up" [22].

This was a major factor that contributed to the very low leave-one-subject out scores that came from athlete testing because the reference orientation was what enabled the Kalman filter to determine the inital conditions for the sensor fusion process. Because these initial conditions were flawed, they produced widely-variable output for the orientation, linear acceleration, and gravity vector readings. The sensor is now manually tared at the beginning of testing an athlete for a particular exercise.

\subsubsection{Magnetometer effects}

The magnetometer in the IMU is misguided due to its proximity to the ferrous metal in the barbell weight collar, the barbell itself, and plates. When the researchers initially got the sensor, they thought about this issue and assumed they would only be using the raw accelerometer and gyroscope values. They moved further along in their research and discovered that the sensor can do "sensor fusion" via a Kalman filter on the IMU itself [21], which could potentially yield more accurate data [93]. Sensor fusion, as detailed in Chapter 2, takes advantage of the fact that the accelerometer, gyroscope, and magnetometer all have different weaknesses and can compensate for those weaknesses by combining them together to obtain a more accurate picture of the motion the IMU is capturing [93]. The resultant outputs of sensor fusion are orientation, linear acceleration, and the direction of gravity. These outputs are dependent on the inputs of all of the sensors 
combined, so one consistently inaccurate reading for one sesnor could affect the end result of all of the sensor fusion outputs.

This is what happened during the first iteration of trainee testing. The researchers did not consider the assumption they made earlier, when considering the sensor's interaction with the context of the application environment (lots of ferrous metals), by using the magnetometer when they moved forward with using the data captured with the sensor fusion feature. The ferrous metal barbells and plates adjust the sensor's perceived orientation by an angle of approximately 30 degrees, but that can hardly be trusted as a constant that stays consistent throughout the testing. The athletic weight room and recreation center use different types of weights and barbells, so the magnetometer-affected data could not be assumed to be off by the same angle in all situations. This change in magnetometer angle so thoroughly undermined the notion of having the sensor know its orientation in space; it was critical for the researchers to find a solution that maximized the distance between the sensor and the ferrous metals that it must still keep track of.

The researchers responded to this issue by putting enough space between the sensor on the collar and plates by using a firm spacer. The spacer puts at minimum 6 inches of non-conductive material between the sensor and the weight collar it will attach to, as seen in Figure 9.1. The distance required between the sensor and weight collar was measured by moving the weight collar closer to the sensor until it had an effect on its perceived orientation as shown on a 3D visualization program provided by the manufacturer of the sensor. Spring clips will be used to secure the lifter's weight, and the sensor weight collar will be attached to the very end of the barbell to prevent interference from the metallic weight lifting plates. 


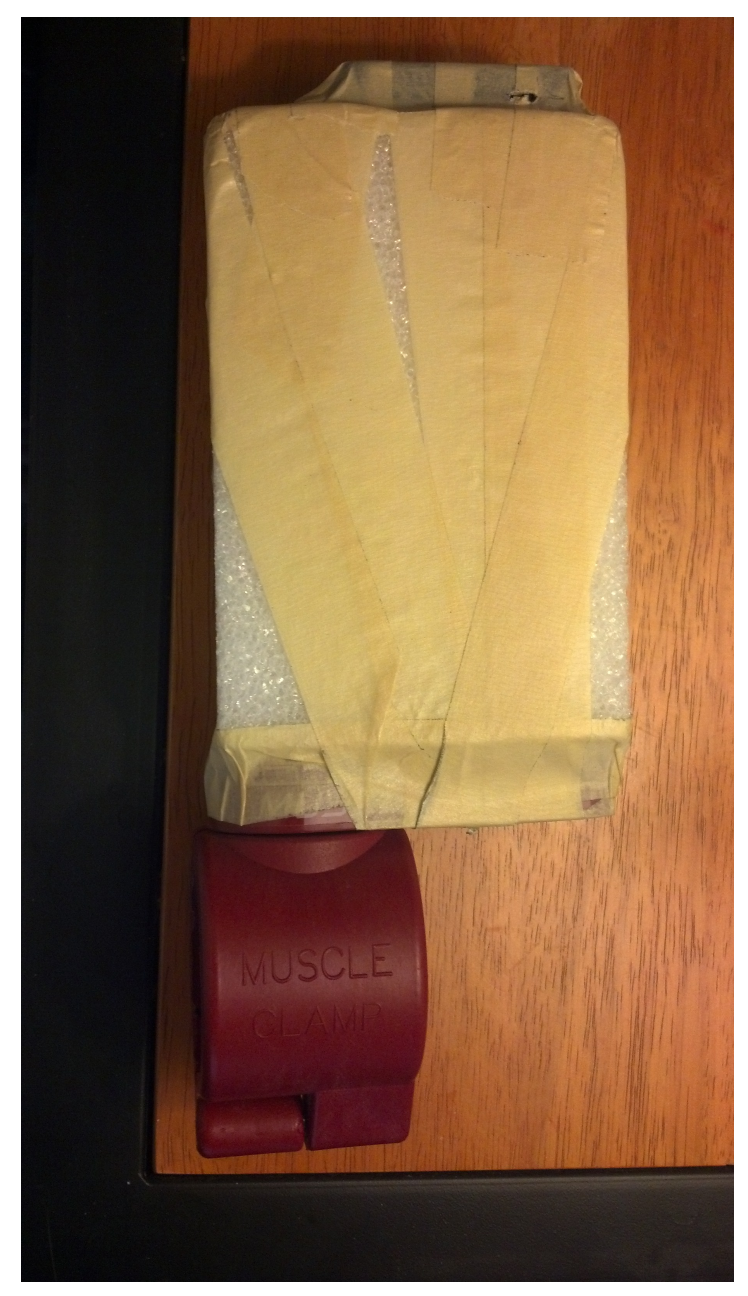

Figure 9.1: The second design of LIFT's sensor packaging

\subsubsection{Euler angles and Gimbal lock}

Another source of inaccurate data was the data format of the orientation output from the Kalman filter. Euler angles, which may be more human readable and understandable than other methods to defining rotation, are prone to what is called "Gimbal lock" [93] when an axis of rotation can be ambiguously defined as another axis of rotation. This is most prominently seen when the pitch goes to 90 degrees, in line with the roll dimension. This causes the sensor to output bad values and could potentially introduce unnecessary variability in the system. 
The researchers considered this before using the Euler angle measurements initially and moved forward with them because they did not anticipate the IMU's pitch to go up to 90 degrees. In testing athletes, the pitch does go to 90 degrees when racking and unracking the weight due to the barbell's weight sleeves that roll independently of the knurling grip midsection.

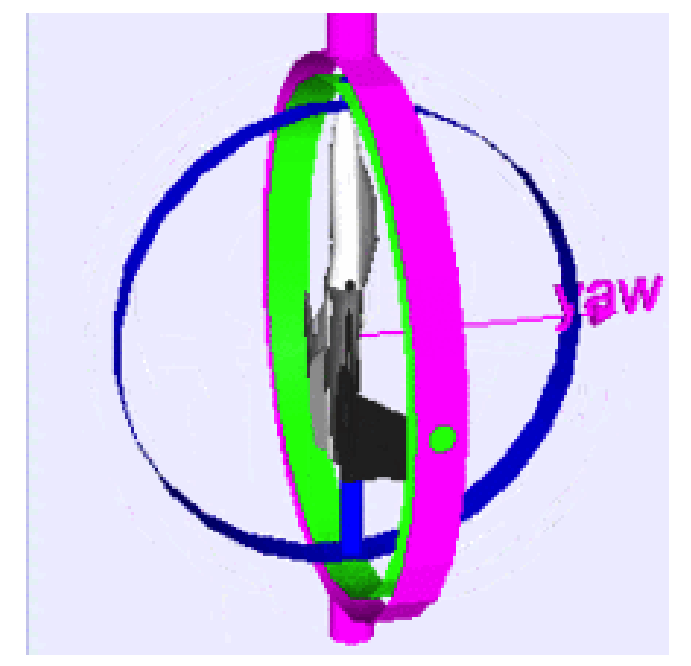

Figure 9.2: The ambiguity between the yaw (pink) and roll (green) degrees of freedom [39]

An alternate data format for defining rotation is the quaternion. The quaternion adds a fourth degree of freedom that does not allow the Gimbal lock to occur where the Euler angles would become ambiguous.

\subsubsection{Data collection rate}

Engineering documentation is sometimes misleading and mislabeled. The researchers discovered at the same time as they were debugging the orientation and magnetometer issues thar they can get a faster data rate from the sensor by using "data streaming", where the sensor periodically sends data, up to $265 \mathrm{~Hz}$ via Bluetooth in theory, to the Android device without needing a request message 
for every data point [21].

This feature was described under the heading "Wired Streaming Mode" in the YEI datasheet, and is the only section on "streaming" there is no corresponding "Wireless Streaming Mode" heading [22]. Until they were debugging sensor orientation code, they did not know that there was such a thing as Bluetooth streaming. The heading "Wired streaming" made it sound like it required a cable to be attached and that precluded their use case.

The researchers are now able to take data at much faster rates at around $100 \mathrm{~Hz}$ with this streaming mode and it has lead to more accurate results than the first iteration of the $20 \mathrm{~Hz}$ message-passing-based collection rate.

\subsubsection{Exercise technique deficiency ambiguity}

Exercise technique deficiencies are sometimes ambiguous because there are multiple deficiencies occurring at once due to the trainee's poor understanding of the proper technique to do in the first place. The researchers have come up with a "deficiency priority" to try and solve this problem. If multiple deficiencies are spotted, then the LIFT analysis tool would just use the label that most directly impacts the core movement of the lift. The "core movement" of a lift is defined as the body parts that are or are closest to the active joints in question for a particular exercise. By having to rework other things for the next data collection iteration, the researchers also had a chance to take what they learned from the first time recording the data set and can make the next data set's labels more accurate. 


\section{CHAPTER 10}

\section{Conclusions}

This research has verified Velloso et al.'s claim that training classifiers to predict technique errors is time-consuming and hard to scale [110]. The classification problem became harder when significantly challenging amounts of weight were tested on participants as well as the many different types of technique deficiencies that are found in barbell exercises, as described in Chapter 2. The classification problem was confounded by erroneous use of the sensor in earlier versions. As those problems became ironed out over time, new ones appeared in the form of significant participant bias towards being undertrained and performing barbell exercises with improper technique as the system was re-tested. This bias towards more novice trainees is just an example of one of the many complex interactions of variables, physical or computational, within the LIFT system.

One of the main takeaways from this research is that although this is a hard problem, there is plenty of commercial potential $[2,3,11,16]$ because this is an important problem for many people. The barriers to teaching people how to exercise safely and effectively with instant feedback in a very widespread fashion have never been lower and are ready for willing innovators to scale over them.

Society has much strength to gain, potential to unlock, many lives to improve, and weight to lose if this research or related commercial ventures continues and finds success. 


\title{
CHAPTER 11
}

\author{
Future Work
}

There are many ways to branch and build off of this groundwork for a on-bar exercise technique feedback system. Below are a few that the researchers would be particularly interested in continuing forward with.

\subsection{Visual feedback}

The LIFT framework aims to put an invaluable weight lifting coaching toolset in the hands of athletes with access to mobile technology. Much of the weight lifting feedback loop can be automated so inexperienced lifters without a personal trainer or weight lifting coach can receive feedback on their weightlifting technique without the hassle of setting up extra equipment. Future iterations of LIFT will be able to visually compare an athlete's bar path with an established standard optimal path for a given lift, or even against other elite-level lifter's bar paths. Another variation of the system could possibly establish an athlete's warm up sets to be the standard which their future sets for that workout should be compared to. If the later sets in the workout start to deviate too much, the system could suggest to the user to decrease the amount of weight they are lifting or stop doing that exercise for that workout. 


\subsection{Repetition detection}

Integrating automated repetition detection algorithms was out of the scope of this research. The focus of this research was to establish an analysis framework for once the data points for each repetition were determined. There is promise in using the magnetometer data for repetition detection, as seen in Figure 11.1.

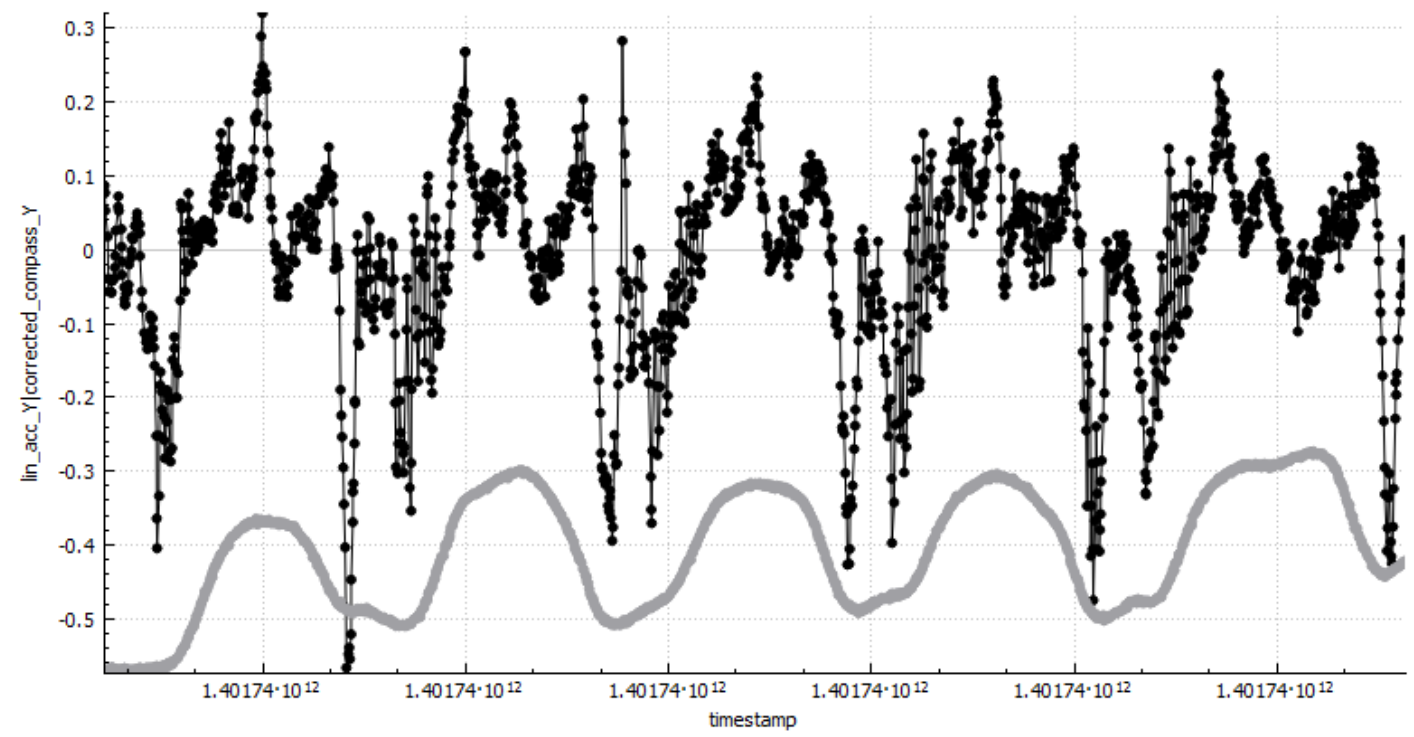

Figure 11.1: Magnetometer measurements (bottom) correlates nicely with when repetitons occured

\subsection{Sensor compatibility}

Compatibility with new movement-tracking devices, such as the Moov [11], would offer new potential for LIFT to more effectively analyze the movement of the barbell if the sensors did more advanced on-board signal filtering and processing. LIFT could even integrate with an SDK that would have built-in position tracking APIs. 


\subsection{Body type clustering}

In the future, the LIFT analysis system's Android app could not only take data points acquired by the sensor into consideration for technique evaluation, but it could also cluster similarly-sized athletes together to account for anatomical variations in bone structure. Athletes who have similar anatomical proportions, such as limb and torso lengths, are more likely to have the same "correct" technique than those who do not. This additional step could help LIFT classify the data the sensor collects more accurately. The anatomical data will be self-reported and could be subject to some amount of error, so ratios of limb lengths can be clustered in addition to the raw limb length values.

This approach was not taken in this research because the researchers' wanted to keep the friction for participation in the study as low as possible.

\subsection{Female study}

The research conducted so far has only been on male student-athletes and recreation center participants. A main concern that was expressed in Section 5.2.1 was that while there would be plenty of female student-athlete participants who do barbell exercises in the athletic weight room, they would be biased towards completing exercises in a "correct" fashion. There is not as large of a corresponding female population in the recreation center that participate in barbell weight lifting exercise, so the data would be heavily skewed towards classifying repetitions as "correct". The researchers could have eventually recorded enough technique deficiencies in the athletic weight room to have relevant results, but unfortunately, this was not feasible within the time frame of when this research 
was to be completed.

11.6 Alternative ways to provide weight lifting feedback

A "golden standard" approach may be how other competing exercise feedback systems are attempting to classify and give exercise technique feedback. The golden standard would classify a repetition outside of a 5-10\% threshold as "incorrect". After it is classified as incorrect, the system would go into more analysis as to specifically how the technique was incorrect and to provide feedback to the user about what they could do differently. 


\section{BIBLIOGRAPHY}

[1] Amiigo. https://amiigo.com/.

[2] Athos Clothing . https://www.liveathos.com/.

[3] Atlas Fitness Tracker. http://atlaswearables.com/.

[4] Barsense app. http://www.barsense.com/.

[5] Basis. http://www.mybasis.com/.

[6] Coach's Eye app. http://www.coachseye.com/.

[7] Fitbit. http://www.fitbit.com/.

[8] Jawbone Up. https://jawbone.com/up.

[9] Kinovea software. http://www.kinovea.org/.

[10] Moov Developers. http://developers.moov.cc/.

[11] Moov Fitness Tracker. http://moov.cc/.

[12] Nike + iPod. https://www.apple.com/ipod/nike/run.html.

[13] Nike+ Fuelband SE. http://www.nike.com/us/en_us/c/nikeplus-fuelband.

[14] Platform Helper app. https://play.google.com/store/apps/details?id=platform.helper.yair.

[15] Polar Flow. https://flow.polar.com/.

[16] Push Strength Tracker. http://www.pushstrength.com/. 
[17] Quantified Self Guide to Self-Tracking Tools. http://quantifiedself.com/guide/.

[18] Sitting facts. http://www.juststand.org/TheFacts/tabid/816/language/enUS/Default.aspx.

[19] The Rise of the Quantified Self in Health Care. http://blogs.wsj.com/venturecapital/2013/08/13/the-rise-of-thequantified-self-in-health-care/.

[20] Withings. http://vitrine.withings.com/.

[21] YEI 3-Space Bluetooth Sensor. http://www.yeitechnology.com/productdisplay/3-space-bluetooth.

[22] YEI 3-Space Bluetooth Sensor User's Manual. http://www.yeitechnology.com/sites/default/files/YEI_3Space_Sensor_Users_Manual_Bluetooth_2.0_r21_26Mar2014.pdf.

[23] Machine Learning 2.8 Random Forests. https://www.youtube.com/watch?v=o7iDkcpOr_g, 62011.

[24] Tutorial: Kalman Filter with MATLAB example part1. https://www.youtube.com/watch?v=FkCT_LV9Syk, 42011.

[25] Chapter 1, traffic detector handbook. http://www.fhwa.dot.gov/publications/research/operations/its/06108/01.cfm, 42012.

[26] Estimating Velocity and Position Using Accelerometers. 102012. 
[27] Android kalman filter accelerometer.

http://www.wikiprohq.com/android-kalman-filter-accelerometer, 52014.

[28] Juul Achten and Asker E Jeukendrup. Heart rate monitoring. Sports medicine, 33(7):517-538, 2003.

[29] Kerem Altun and Billur Barshan. Human activity recognition using inertial/magnetic sensor units. In Human Behavior Understanding, pages 38-51. Springer, 2010.

[30] Marc Bächlin, Kilian Förster, and Gerhard Tröster. SwimMaster: A wearable assistant for swimmer. In Ubicomp '09: Proceedings of the 11th international conference on Ubiquitous computing, pages 215224, New York, NY, USA, September 2009. ACM.

[31] James Balmer, Rc Richard Davison, and Steve R Bird. Peak power predicts performance power during an outdoor 16.1-km cycling time trial. Medicine and Science in Sports and Exercise, 32(8):1485-1490, 2000.

[32] K.A. Bonnet. Systems and methods for comprehensive human movement analysis, April 15 2014. US Patent 8,698,888.

[33] Leo Breiman. Random forests. Machine learning, 45(1):5-32, 2001.

[34] Jeffrey P Broker. Cycling power: road and mountain. High-Tech Cycling: The science of riding faster, pages 147-174, 2003.

[35] Yongyao Cai, Yang Zhao, Xianfeng Ding, and James Fennelly. Magnetometer basics for mobile phone applications. 
http://www.electronicproducts.com/Sensors_and_Transducers/ Sensors_and_Transducers/Magnetometer_basics_for_mobile_phone_applications.aspx, 12012.

[36] Andrew J. Callaway and Jon E. Cobb. Linear Acceleration Measurement Utilizing Inter-Instrument Synchronization: A Comparison between Accelerometers and Motion-Based Tracking Approaches. Measurement in Physical Education and Exercise Science, 16(2):151-163, 2012.

[37] John F Caruso, Nathan M Olson, Skyler T Taylor, Jessica R McLagan, Catherine M Shepherd, Jake A Borgsmiller, Melissa L Mason, Rebekah R Riner, Laura Gilliland, and Shawn Grisewold. Front squat data reproducibility collected with a triple-axis accelerometer. The Journal of Strength 83 Conditioning Research, 26(1):40-46, 2012.

[38] Paul Chek. How to eat, move and be healthy. CHEK Institute, San Diego, CA, 2004.

[39] Wikimedia Commons. Gimbal lock, 2011. File: Gimbal_lock.jpg.

[40] Rachel Cooper, Diana Kuh, Rebecca Hardy, Mortality Review Group, et al. Objectively measured physical capability levels and mortality: systematic review and meta-analysis. BMJ: British Medical Journal, $341,2010$.

[41] Dekhtyar, Alexander. Data Mining: Classication/Supervised Learning. http://users.csc.calpoly.edu/ dekhtyar/466Spring2012/lectures/lec05.466.pdf, 2012. 
[42] Michael J Duffey and John H Challis. Vertical and lateral forces applied to the bar during the bench press in novice lifters. The Journal of Strength \& Conditioning Research, 25(9):2442-2447, 2011.

[43] M. Duncan, S. Parker, C. Worringham, P. Condie, A. Barriskill, B. Curcic, J. Jasiewicz, and T. Parker. Motion Monitoring and Analysis System, October 25 2007. US Patent App. 10/562,858.

[44] Avery D Faigenbaum and Gregory D Myer. Resistance training among young athletes: safety, efficacy and injury prevention effects. British journal of sports medicine, 44(1):56-63, 2010.

[45] John Garhammer. Biomechanical Profiles of Olympic Weightlifters. International Journal of Sport Biomechanics, 1(2), 1985.

[46] John Geen and David Krakauer. New imems@ angular-rate-sensing gyroscope. Analog Dialogue, 37(3):1-4, 2003.

[47] Pierre Geurts, Damien Ernst, and Louis Wehenkel. Extremely randomized trees. Machine learning, 63(1):3-42, 2006.

[48] J. Giuffrida. Movement disorder monitoring system and method, May 29 2012. US Patent 8,187,209.

[49] D. Gordon, S.L. Mullane, P.P. Conway, and A.A. West. Development of a Novel System for Monitoring Strength and Conditioning in Elite Athletes. Procedia Engineering, 34(0):496 - 501, 2012. Engineering of Sport Conference 2012 .

[50] Vivienne Gray. The framing of Socrates: the literary interpretation of Xenophon's Memorabilia, volume 79. Franz Steiner Verlag, 1998. 
[51] Giuliana Grimaldi and Mario Manto. Neurological tremor: Sensors, signal processing and emerging applications. Sensors, 10(2):1399-1422, 2010.

[52] John J. Guiry, Pepijn van de Ven, and John Nelson. Multi-Sensor Fusion for Enhanced Contextual Awareness of Everyday Activities with Ubiquitous Devices. Sensors, 14(3):5687-5701, 2014.

[53] Mohammad Ashfak Habib, Mas S Mohktar, Shahrul Bahyah Kamaruzzaman, Kheng Seang Lim, Tan Maw Pin, and Fatimah Ibrahim. Smartphone-based solutions for fall detection and prevention: challenges and open issues. Sensors, 14(4):7181-7208, 2014.

[54] Mats Hagberg. Muscular endurance and surface electromyogram in isometric and dynamic exercise. Journal of Applied Physiology, 51(1):17,1981

[55] Fred Hamprecht. $\quad 6.3$ Random Forest. https://www.youtube.com/watch?v=gdnIqGbqiYs, 112012.

[56] Hagen Hartmann, Klaus Wirth, and Markus Klusemann. Analysis of the load on the knee joint and vertebral column with changes in squatting depth and weight load. Sports Medicine, 43(10):993-1008, 2013.

[57] Robert Hurling, Michael Catt, Marco De Boni, William Bruce Fairley, Tina Hurst, Peter Murray, Alannah Richardson, and Singh Jaspreet Sodhi. Using Internet and Mobile Phone Technology to Deliver an Automated Physical Activity Program: Randomized Controlled Trial. J Med Internet Res, 9(2):e7, Apr 2007. 
[58] Asker Jeukendrup and Adrie Van Diemen. Heart rate monitoring during training and competition in cyclists. Journal of Sports Sciences, 16(sup1):91-99, 1998.

[59] Hao Jiang and Jason O. Hallstrom. Fast, Accurate Event Classification on Resource-Lean Embedded Sensors. ACM Trans. Auton. Adapt. Syst., 8(2):11:1-11:22, July 2013.

[60] Hemant Khandelwal. Snapshot of the wearable device market. http://www.crunchyfriday.com/snapshot-of-wearable-device-market/, 12014 .

[61] Gihan Kuruppu, S. R. Kodituwakku, and U. A. J. Pinidiyaarachchi. High Speed Motion Tracking for Weightlifting based on Correlation Coefficient Template Matching.

[62] Zhi Li and Guanglie Zhang. A gait recognition system for rehabilitation based on wearable micro inertial measurement unit. In Robotics and Biomimetics (ROBIO), 2011 IEEE International Conference on, pages 1678-1682, Dec 2011.

[63] Wade A Lillegard, Eugene W Brown, Daniel J Wilson, Ruben Henderson, and Evelyn Lewis. Efficacy of strength training in prepubescent to early postpubescent males and females: effects of gender and maturity. Developmental Neurorehabilitation, 1(3):147-157, 1997.

[64] Z. Lin, M. Zecca, S. Sessa, L. Bartolomeo, H. Ishii, K. Itoh, and A. Takanishi. Development of an ultra-miniaturized inertial measurement unit WB-3 for human body motion tracking. In System In- 
tegration (SII), 2010 IEEE/SICE International Symposium on, pages 414-419, Dec 2010.

[65] Henrik Linusson, Robin Rudenwall, and Andreas Olausson. Random forest och glesa datarespresentationer. University of Bor Âs/School of Business and IT, 2012.

[66] Michael Marschollek, Matthias Gietzelt, Mareike Schulze, Martin Kohlmann, Bianying Song, and Klaus-Hendrik Wolf. Wearable sensors in healthcare and sensor-enhanced health information systems: all our tomorrows? Healthcare informatics research, 18(2):97-104, 2012.

[67] James C Martin, Douglas L Milliken, John E Cobb, Kevin L McFadden, and Andrew R Coggan. Validation of a mathematical model for road cycling power. Journal of applied biomechanics, 14:276-291, 1998.

[68] Jerry L Mayhew, Blair D Johnson, Michael J LaMonte, Dirk Lauber, and Wolfgang Kemmler. Accuracy of prediction equations for determining one repetition maximum bench press in women before and after resistance training. The Journal of Strength \& Conditioning Research, $22(5): 1570-1577,2008$.

[69] Ryan S McGinnis. Advancing Applications of IMUs in Sports Training and Biomechanics. PhD thesis, University of California, 2013.

[70] J. McNames, P.M.R. Aboy, and A. Greenberg. Complete integrated system for continuous monitoring and analysis of movement disorders, March 25 2010. US Patent App. 12/565,697.

[71] Mehdi. Fractional Plates. http://stronglifts.com/5x5/\#Fractional_Plates. 
[72] Mehdi. Madcow 5 x 5: Another Simple Routine to Get Stronger After StrongLifts 5 x 5 . http://stronglifts.com/madcow-5x5-trainingprograms/.

[73] Mehdi. Microloading Solutions. http://stronglifts.com/madcow/Topics/Microloading.htm.

[74] Brandon J Miller and Fadi A Fathallah. Body-fixed orientation sensors for trunk motion sensing. In Proceedings of the Human Factors and Ergonomics Society Annual Meeting, volume 51, pages 933-937. SAGE Publications, 2007.

[75] George A Miller. The magical number seven, plus or minus two: some limits on our capacity for processing information. Psychological review, 63(2):81, 1956.

[76] A. Moeller, L. Roalter, S. Diewald, M. Kranz, N. Hammerla, P. Olivier, and T. Ploetz. Gymskill: A personal trainer for physical exercises. In Proceedings of the International Conference on Pervasive Computing and Communications, 2012.

[77] Isabel S Moore, Andrew M Jones, and Sharon J Dixon. Mechanisms for improved running economy in beginner runners. Medicine 83 Science in Sports \& Exercise, 2012.

[78] Bobak Mortazavi, Mohammad Pourhomayoun, Gabriel Alsheikh, Nabil Alshurafa, Sunghoon Ivan Lee, and Majid Sarrafzadeh. Determining the Single Best Axis for Exercise Repetition Recognition and Counting with SmartWatches. 
[79] Sam K Morton, James R Whitehead, Ronald H Brinkert, and Dennis J Caine. Resistance training vs. static stretching: effects on flexibility and strength. The Journal of Strength 83 Conditioning Research, 25(12):3391-3398, 2011.

[80] Nathaniel Mott. Fitness Tech Needs to Pick Up the Pace. http://pando.com/2012/05/21/fitness-tech-needs-to-pick-up-thepace/.

[81] SL Mullane, N Chakravorti, PP Conway, and AA West. Design and implementation of a user-centric swimming performance monitoring tool. Proceedings of the Institution of Mechanical Engineers, Part P: Journal of Sports Engineering and Technology, 225(4):213-229, 2011.

[82] Bijan Najafi, Deena Horn, Samuel Marclay, Ryan T Crews, Stephanie Wu, and James S Wrobel. Assessing postural control and postural control strategy in diabetes patients using innovative and wearable technology. Journal of diabetes science and technology, 4(4):780-791, 2010.

[83] Shahram Lenjan Nejadian, Mostafa Rostami, and Abolghasem Naghash. Cost evaluation of different snatch trajectories by using dynamic programming method. Procedia Engineering, 2(2):2563-2567, 2010.

[84] G Neumayr, R Pfister, G Mitterbauer, H Gaenzer, W Sturm, and H Hoertnagl. Heart rate response to ultraendurance cycling. British journal of sports medicine, 37(1):89-90, 2003.

[85] I. Newton, A. Motte, and J. Machin. The Mathematical Principles of 
Natural Philosophy. Number v. 1 in The Mathematical Principles of Natural Philosophy. B. Motte, 1729.

[86] F. Pedregosa, G. Varoquaux, A. Gramfort, V. Michel, B. Thirion, O. Grisel, M. Blondel, P. Prettenhofer, R. Weiss, V. Dubourg, J. Vanderplas, A. Passos, D. Cournapeau, M. Brucher, M. Perrot, and E. Duchesnay. Scikit-learn: Machine learning in Python. Journal of Machine Learning Research, 12:2825-2830, 2011.

[87] Igor Pernek, Karin Anna Hummel, and Peter Kokol. Exercise Repetition Detection for Resistance Training Based on Smartphones. Personal Ubiquitous Comput., 17(4):771-782, April 2013.

[88] Rodrigo Prez, rsula Costa, Marc Torrent, Javier Solana, Eloy Opisso, Csar Cceres, Josep M. Tormos, Josep Medina, and Enrique J. Gmez. Upper limb portable motion analysis system based on inertial technology for neurorehabilitation purposes. Sensors, 10(12):10733-10751, 2010.

[89] John Ross Quinlan. C4.5: programs for machine learning, volume 1. Morgan Kaufmann, 1993.

[90] Mark Rippetoe. Starting Strength. The Aasgaard Company.

[91] Jari-Pekka Rontu, Manne I Hannula, Sami Leskinen, Vesa Linnamo, and Jukka A Salmi. One-repetition maximum bench press performance estimated with a new accelerometer method. The Journal of Strength E3 Conditioning Research, 24(8):2018-2025, 2010.

[92] Dan Rowinski. How Google's Fitness API Will Buff You Up. 
http://readwrite.com/2014/01/17/how-google-fitness-api-contextualsensor-data-android-users.

[93] David Sachs. Sensor Fusion on Android Devices: A Revolution in Motion Processing. https://www.youtube.com/watch?v=C7JQ7Rpwn2k, 8 2010.

[94] Kimi Sato and Paul Fleschler. A new approach to measure weightlifting performance "introducing an accelerometer".

[95] Kimitake Sato, Paul Fleschler, and W Sands. Barbell acceleration analysis on various intensities of weightlifting. In ISBS-Conference Proceedings Archive, volume 1, 2009.

[96] Kimitake Sato, William A Sands, and Michael H Stone. The reliability of accelerometry to measure weightlifting performance. Sports Biomechanics, 11(4):524-531, 2012.

[97] Kimitake Sato, Sarah L Smith, and William A Sands. Validation of an accelerometer for measuring sport performance. The Journal of Strength $\& 3$ Conditioning Research, 23(1):341-347, 2009.

[98] M. Schulze, Tsung-Han Liu, Jiang Xie, Wu Zhang, Klaus-Hendrik Wolf, T. Calliess, H. Windhagen, and M. Marschollek. Unobtrusive ambulatory estimation of knee joint angles during walking using gyroscope and accelerometer data - a preliminary evaluation study. In Biomedical and Health Informatics (BHI), 2012 IEEE-EMBS International Conference on, pages 559-562, Jan 2012.

[99] Christian Seeger, Alejandro Buchmann, and Kristof Van Laerhoven. myHealthAssistant: A Phone-based Body Sensor Network That Cap- 
tures the Wearer's Exercises Throughout the Day. In Proceedings of the 6th International Conference on Body Area Networks, BodyNets '11, pages 1-7, ICST, Brussels, Belgium, Belgium, 2011. ICST (Institute for Computer Sciences, Social-Informatics and Telecommunications Engineering).

[100] A. Shamaie. Tracking bimanual movements, September 11 2008. US Patent App. 11/932,766.

[101] T. Shany, S.J. Redmond, M.R. Narayanan, and N.H. Lovell. SensorsBased Wearable Systems for Monitoring of Human Movement and Falls. Sensors Journal, IEEE, 12(3):658-670, March 2012.

[102] Jason Shea. Fatigue, recovery, and supercompensation. http://www.teamunify.com/cseksc/__doc_-/ Shea_FatigueRecovery Supercompensation-2.pdf.

[103] A.V.W. Smith, A.I. Sutherland, A. Lemoine, and S. Mcgrath. Hand gesture recognition system and method, October 3 2000. US Patent $6,128,003$.

[104] Z.S. Sobolewski. Intelligent sport shoe system, June 18 2013. US Patent $8,467,979$.

[105] Gábor Sörös, Florian Daiber, and Tomer Weller. Cyclo: A personal bike coach through the glass. In SIGGRAPH Asia 2013 Symposium on Mobile Graphics and Interactive Applications, SA '13, pages 99:199:4, New York, NY, USA, 2013. ACM.

[106] K Starrett. Becoming A Supple Leopard. Victory Belt Publishing, 2013. 
[107] MJ Thompson, M Li, and DA Horsley. Low power 3-axis lorentz force navigation magnetometer. In Micro Electro Mechanical Systems (MEMS), 2011 IEEE 24th International Conference on, pages 593596. IEEE, 2011.

[108] Alexander A Trusov. Overview of MEMS Gyroscopes: History, Principles of Operations, Types of Measurements. 2011.

[109] Y. Varatharajah, N. Karunathilaka, M. Rismi, S. Kotinkaduwa, and D. Dias. Body area sensor network for evaluating fitness exercise. In Wireless and Mobile Networking Conference (WMNC), 2013 6th Joint IFIP, pages 1-8, April 2013.

[110] Eduardo Velloso, Andreas Bulling, Hans Gellersen, Wallace Ugulino, and Hugo Fuks. Qualitative activity recognition of weight lifting exercises. In Proceedings of the 4th Augmented Human International Conference, pages 116-123. ACM, 2013.

[111] Daniel Vlasic, Rolf Adelsberger, Giovanni Vannucci, John Barnwell, Markus Gross, Wojciech Matusik, and Jovan Popović. Practical Motion Capture in Everyday Surroundings. ACM Trans. Graph., 26(3), July 2007.

[112] Patrick L Walter. The history of the accelerometer. Sound and vibration, 31(3):16-23, 1997.

[113] Bill Wilson. $\quad$ Induction of Decision Trees Notes. http://www.cse.unsw.edu.au/billw/ cs9414/notes/ml/06prop/id3/id3.html, 2008.

[114] Gary Wolf. Quantified Self. http://quantifiedself.com/about/. 
[115] Xiaoyi Zhang, Ming-Chun Huang, Fengbo Ren, Wenyao Xu, Nan Guan, and Wang Yi. Proper running posture guide: A wearable biomechanics capture system. In Proceedings of the 8th International Conference on Body Area Networks, BodyNets '13, pages 83-89, ICST, Brussels, Belgium, Belgium, 2013. ICST (Institute for Computer Sciences, Social-Informatics and Telecommunications Engineering). 